TRANSACTIONS OF THE

AMERICAN MATHEMATICAL SOCIETY

Volume 353, Number 7 , Pages 2557-2599

S 0002-9947(01)02827-6

Article electronically published on March 16, 2001

\title{
DISCRETE SERIES CHARACTERS AS LIFTS FROM TWO-STRUCTURE GROUPS
}

\author{
REBECCA A. HERB
}

\begin{abstract}
Let $G$ be a connected reductive Lie group with a relatively compact Cartan subgroup. Then it has relative discrete series representations. The main result of this paper is a formula expressing relative discrete series characters on $G$ as "lifts" of relative discrete series characters on smaller groups called two-structure groups for $G$. The two-structure groups are connected reductive Lie groups which are locally isomorphic to the direct product of an abelian group and simple groups which are real forms of $S L(2, \mathbf{C})$ or $S O(5, \mathbf{C})$. They are not necessarily subgroups of $G$, but they "share" the relatively compact Cartan subgroup and certain other Cartan subgroups with $G$. The character identity is similar to formulas coming from endoscopic lifting, but the twostructure groups are not necessarily endoscopic groups, and the characters lifted are not stable. Finally, the formulas are valid for non-linear as well as linear groups.
\end{abstract}

\section{INTRODUCTION}

Let $G$ be a connected reductive Lie group with a relatively compact Cartan subgroup. Then it has relative discrete series representations. The main result of this paper is a formula expressing relative discrete series characters on $G$ as "lifts" of relative discrete series characters on smaller groups called two-structure groups for $G$. The two-structure groups are connected reductive Lie groups which are locally isomorphic to the direct product of an abelian group and simple groups which are real forms of $S L(2, \mathbf{C})$ or $S O(5, \mathbf{C})$. They are not necessarily subgroups of $G$, but they "share" the relatively compact Cartan subgroup and certain other Cartan subgroups with $G$. The character identity is similar to formulas coming from endoscopic lifting, but the two-structure groups are not necessarily endoscopic groups, and the characters lifted are not stable. Finally, the formulas are valid for non-linear as well as linear groups.

Two-structures were used in [5] to prove identities for the constants occuring in Harish-Chandra's discrete series character formulas. In these formulas the character is restricted to the connected component of a Cartan subgroup, and the twostructures used are those corresponding to a set of real roots determined by the connected component. Thus there is a different formula for each connected component of each Cartan subgroup. These formulas are used in this paper to prove the lifting formula. The main technical difficulty is to see that all these formulas can

Received by the editors June 1, 1999.

2000 Mathematics Subject Classification. Primary 22E30, 22 E45.

Supported in part by NSF Grant DMS 9705645.

(C)2001 American Mathematical Society 
be combined into a formula which is uniform on all Cartan subgroups and given in terms of the two-structures of the full root system of $G$.

Two-structures for $G$ are defined as follows. Let $B$ be a relatively compact Cartan subgroup of $G$, that is a Cartan subgroup that is compact modulo the center of $G$. Let $g$ and $\underline{b}$ denote the real Lie algebras of $G$ and $B$ respectively, and let $\Phi=\Phi\left(\underline{g}_{\mathbf{C}}, \underline{b}_{\mathbf{C}}\right)$ denote the set of roots of $\underline{g}_{\mathbf{C}}$ with respect to $\underline{b}_{\mathbf{C}}$. Let $\varphi$ be a root subsystem of $\Phi$, that is a subset of $\Phi$ which is closed under its own reflections, and hence is a root system with the same inner product as that of $\Phi$. Then $\varphi$ is called a two-structure for $\Phi$ if it satisfies the following two properties.

(i) Every irreducible factor of $\varphi$ is of type $A_{1}$ or $B_{2} \simeq C_{2}$.

(ii) Let $\varphi^{+}$be any choice of positive roots for $\varphi$. Then if $w$ is in the Weyl group of $\Phi$ with $w \varphi^{+}=\varphi^{+}$we have $\operatorname{det} w=1$.

We let $\mathcal{T}(\Phi)$ denote the set of all two-structures for $\Phi$.

The sets $\mathcal{T}(\Phi)$ for irreducible $\Phi$ can be described as follows. If $\Phi$ has one root length or is of type $G_{2}$, then $\mathcal{T}(\Phi)$ consists of all root subsystems of $\Phi$ of type $A_{1}^{k}$ where $k$ is the size of a maximal set of orthogonal roots in $\Phi$. If $\Phi$ is of type $B_{2 k}, C_{2 k}, k \geq 1$, or $F_{2 k}, k=2$, then $\mathcal{T}(\Phi)$ consists of all root subsystems of $\Phi$ of type $B_{2}^{k}$. Finally, if $\Phi$ is of type $B_{2 k+1}, C_{2 k+1}, k \geq 1$, then $\mathcal{T}(\Phi)$ consists of all root subsystems of $\Phi$ of type $B_{2}^{k} \times A_{1}$.

The groups $G_{\varphi}$ are defined as follows. Fix $\varphi \in \mathcal{T}(\Phi)$. It is a root subsystem of $\Phi$. However it need not be closed in $\Phi$ under addition, and so does not necessarily correspond to a subalgebra of $\underline{g}$. Write $\varphi=\varphi_{1} \cup \ldots \cup \varphi_{k}$ for its decomposition into irreducible factors. Then each $\varphi_{i}, 1 \leq i \leq k$, is closed under addition in $\Phi$, and so corresponds naturally to a simple Lie subalgebra $\underline{g}_{i}$ of $\underline{g}$. This subalgebra has the property that if $\underline{b}_{i}=\underline{b} \cap \underline{g}_{i}$, then $\underline{b}_{i}$ is a Cartan subalgebra of $\underline{g}_{i}$ and $\Phi\left(\left(\underline{g}_{i}\right)_{\mathbf{C}},\left(\underline{b}_{i}\right)_{\mathbf{C}}\right)=\varphi_{i}, 1 \leq i \leq k$. We also define

$$
\underline{g}_{0}=\underline{b}_{0}=\{H \in \underline{b}: \alpha(H)=0 \forall \alpha \in \varphi\} .
$$

Then

$$
\sum_{i=0}^{k} \underline{b}_{i}=\underline{b} .
$$

Let $G_{i}$ be the connected subgroup of $G$ corresponding to $\underline{g}_{i}, B_{i}=\exp \left(\underline{b}_{i}\right)=$ $B \cap G_{i}, 0 \leq i \leq k$. Let $G_{0} \times G_{1} \times \ldots \times G_{k}$ denote the abstract direct product of the groups $G_{i}, 0 \leq i \leq k$. Since $B_{i} \subset B, 0 \leq i \leq k$, and $B$ is abelian, the mapping $f: B_{0} \times \ldots \times B_{k} \rightarrow B$ given by

$$
f\left(b_{0}, \ldots, b_{k}\right)=b_{0} \ldots b_{k}, \quad b_{i} \in B_{i}, 0 \leq i \leq k,
$$

is a group homomorphism. Let $Z_{B}$ denote the kernel of this homomorphism. It is easy to prove that $Z_{B}$ is a central subgroup of $G_{0} \times \ldots \times G_{k}$. Define

$$
G_{\varphi}=\left(G_{0} \times \ldots \times G_{k}\right) / Z_{B} \text { and } B_{\varphi}=\left(B_{0} \times \ldots \times B_{k}\right) / Z_{B} .
$$

Then $G_{\varphi}$ is a connected reductive Lie group with relatively compact Cartan subgroup $B_{\varphi}$, and the mapping $f_{B}: B_{\varphi} \rightarrow B$ induced by $f$ is an isomorphism.

Let $\underline{g}_{\varphi}$ and $\underline{b}_{\varphi}$ denote the Lie algebras of $G_{\varphi}$ and $B_{\varphi}$ respectively. Then

$$
\Phi\left(\left(\underline{g}_{\varphi}\right)_{\mathbf{C}},\left(\underline{b}_{\varphi}\right)_{\mathbf{C}}\right)=\varphi
$$


Since all irreducible factors of $\varphi$ are of type $A_{1}$ or $B_{2}, G_{\varphi}$ is locally isomorphic to the direct product of an abelian group and simple groups which are real forms of $S L(2, \mathbf{C})$ or $S O(5, \mathbf{C})$. Note that

$$
\underline{g}_{\varphi}=\bigoplus \sum_{i=0}^{k} \underline{g}_{i}
$$

can be identified with a subspace, but not necessarily a subalgebra, of $\underline{g}$, because elements from the different subalgebras $\underline{g}_{i}$ do not necessarily commute with each other as elements of $\underline{g}$. This is because, although roots in different irreducible factors of $\varphi$ are orthogonal to each other, they need not be strongly orthogonal as elements of $\Phi$. Thus $G_{\varphi}$ can not necessarily be embedded as a subgroup of $G$.

If $\Phi$ is spanned by orthogonal roots, then each $\varphi \in \mathcal{T}(\Phi)$ has the same rank as $\Phi$. Thus if $\Phi$ is spanned by orthogonal roots and $G$ is semisimple, $\underline{g}_{0}=\underline{b}_{0}=\{0\}$, so that $G_{\varphi}$ is also semisimple. If $\Phi$ is not spanned by orthogonal roots, then $\operatorname{rank} \varphi<\operatorname{rank} \Phi$, and so in this case $\underline{g}_{0}=\underline{b}_{0}$ is always non-trivial. Thus even if $G$ is semisimple, it is possible that $G_{\varphi}$ is not. This is why we work with the class of reductive groups.

Let $\mathcal{S O}(\Phi)$ denote the set of all subsets of $\Phi$ consisting of strongly orthogonal noncompact roots. Each $S \in \mathcal{S O}(\Phi)$ corresponds to a Cartan subgroup $H_{S}$ of $G$. Its Lie algebra satisfies $\left(\underline{h}_{S}\right)_{\mathbf{C}}=c_{S}\left(\underline{b}_{\mathbf{C}}\right)$ where $c_{S}$ is the Cayley transform associated to $S$. Further, $H_{S}$ can be decomposed as $H_{S}=T_{S} A_{S}$ where $A_{S}$ is a split group and $T_{S}$ is compact modulo the center of $G$ and has identity component $T_{S}^{0} \subset B$. While $T_{S}$ need not be abelian when $G$ is not linear, $T_{S}^{1}=T_{S} \cap B$ is always abelian, since $B$ is. We define $H_{S}^{1}=T_{S}^{1} A_{S}$. If $\gamma \in G^{\prime}$ is any regular semisimple element of $G$, then there are $S \in \mathcal{S} O(\Phi)$ and $h \in H_{S}^{1}$ (not unique) such that $\gamma=x h x^{-1}$ for some $x \in G$.

Now for $\varphi \in \mathcal{T}(\Phi)$, we let $\mathcal{S O}(\varphi)$ denote the set of all subsets of $\varphi$ consisting of noncompact roots which are strongly orthogonal in $\varphi$. Each $S \in \mathcal{S O}(\varphi)$ corresponds as above to a Cartan subgroup $H_{\varphi, S}=T_{\varphi, S} A_{\varphi, S}$ of $G_{\varphi}$. In this case we always have $T_{\varphi, S} \subset B_{\varphi}$, so that $H_{\varphi, S}^{1}=H_{\varphi, S}$.

Let $\mathcal{S} O(\Phi, \varphi)=\mathcal{S O}(\Phi) \cap \mathcal{S O}(\varphi)$. Then for $S \in \mathcal{S} O(\Phi, \varphi)$ we have Cartan subgroups $H_{S}$ of $G$ and $H_{\varphi, S}$ of $G_{\varphi}$. If $S_{i}=S \cap \varphi_{i}, 1 \leq i \leq k$, then

$$
H_{\varphi, S}=\left(H_{0, S_{0}} \times H_{1, S_{1}} \times \ldots \times H_{k, S_{k}}\right) / Z_{B}
$$

where $H_{0, S_{0}}=B_{0}$ and $H_{i, S_{i}}$ is the Cartan subgroup of $G_{i}$ corresponding to $S_{i}, 1 \leq$ $i \leq k$. Now $H_{i, S_{i}} \subset H_{S}^{1}, 0 \leq i \leq k$, and $\left(h_{0}, h_{1}, \ldots, h_{k}\right) \mapsto h_{0} h_{1} \ldots h_{k}, h_{i} \in H_{i, S_{i}}, 0 \leq$ $i \leq k$, factors through $H_{\varphi, S}$ to give an isomorphism $f_{S}$ of $H_{\varphi, S}$ onto $H_{S}^{1}$.

The isomorphisms $f_{S}: H_{\varphi, S} \rightarrow H_{S}^{1}, S \in \mathcal{S O}(\Phi, \varphi)$, can be used to define a correspondence of orbits between $G_{\varphi}$ and $G$. For $x \in G_{\varphi}$ and $g \in G$, let $\mathcal{O}_{\varphi}(x)$ and $\mathcal{O}_{G}(g)$ denote the orbits of $x$ in $G_{\varphi}$ and $g$ in $G$ respectively. Let $x \in G_{\varphi}^{\prime}$, the set of regular semisimple elements in $G_{\varphi}$. Then there exist $S \in \mathcal{S O}(\varphi)$ and $h \in H_{\varphi, S}$ (not unique) such that $\mathcal{O}_{\varphi}(x)=\mathcal{O}_{\varphi}(h)$. We will say that $\mathcal{O}_{\varphi}(x)$ is a good orbit in $G_{\varphi}^{\prime}$ if $S \in \mathcal{S O}(\Phi, \varphi)$, that is the roots in $S$ are strongly orthogonal, not only in $\varphi$, but also in $\Phi$. In the case that $\mathcal{O}_{\varphi}(x)=\mathcal{O}_{\varphi}(h), h \in H_{\varphi, S}$, is a good orbit, we have the Cartan subgroup $H_{S}$ of $G$ and the isomorphism $f_{S}: H_{\varphi, S} \rightarrow H_{S}^{1}$. We define

$$
F_{\varphi}\left(\mathcal{O}_{\varphi}(x)\right)=\mathcal{O}_{G}\left(f_{S}(h)\right) .
$$

We prove in $\S 4$ that this definition is independent of the choice of $S \in \mathcal{S O}(\varphi)$ and $h \in H_{\varphi, S}$ with $\mathcal{O}_{\varphi}(x)=\mathcal{O}_{\varphi}(h)$. 
Now that we have a correspondence of orbits between $G_{\varphi}$ and $G$ we can define lifting from $G_{\varphi}$ to $G$. For $x \in G$, write $\operatorname{det}\left(t-1+A d_{G}(x)\right)=D_{G}(x) t^{n}+$ terms of higher degree, where $t$ is an indeterminate. Then $x$ is regular just in case $D_{G}(x) \neq 0$, and we write $G^{\prime}$ for the set of regular elements. We also write $D_{\varphi}(x)=D_{G_{\varphi}}(x), x \in$ $G_{\varphi}$, for the corresponding function on $G_{\varphi}$. Let $S \in \mathcal{S O}(\Phi, \varphi)$ and $h^{\prime} \in H_{\varphi, S}$ such that $h=f_{S}\left(h^{\prime}\right) \in H_{S}^{1} \cap G^{\prime}$. Then we define

$$
D_{\varphi}^{G}\left(h^{\prime}\right)=\left|D_{\varphi}\left(h^{\prime}\right)\right|^{\frac{1}{2}}\left|D_{G}(h)\right|^{-\frac{1}{2}} .
$$

Then $D_{\varphi}^{G}$ extends uniquely to a class function on the union of good orbits $\mathcal{O}_{\varphi}$ of $G_{\varphi}^{\prime}$ such that $F_{\varphi}\left(\mathcal{O}_{\varphi}\right)$ is a regular orbit in $G$.

We can now define a lifting from $G_{\varphi}$ to $G$ as follows. For $g \in G^{\prime}$, let $X_{\varphi}(g)$ denote a complete set of representatives for the good $G_{\varphi}$ orbits which map to $\mathcal{O}_{G}(g)$ under the orbit correspondence $F_{\varphi}$. Let $\Theta$ be a class function defined on $G_{\varphi}^{\prime}$. Now for $g \in G^{\prime}$, we define

$$
\left(\operatorname{Lift}_{\varphi}^{G} \Theta\right)(g)=\sum_{h^{\prime} \in X_{\varphi}(g)} D_{\varphi}^{G}\left(h^{\prime}\right) \Theta\left(h^{\prime}\right) .
$$

Since $D_{\varphi}^{G}$ and $\Theta$ are constant on $G_{\varphi}$ orbits, $\left(\operatorname{Lift}_{\varphi}^{G} \Theta\right)(g)$ does not depend on the choice of $X_{\varphi}(g)$. It defines a class function on $G^{\prime}$.

We now turn to discrete series characters. Let $L_{B}$ denote the set of all $\tau \in i \underline{b}^{*}$ such that $e^{\tau}$ gives a well-defined character of $B$, and let $\rho_{G}$ denote the half-sum of positive roots in $\Phi$. Then $\lambda \in i \underline{b}^{*}$ is a discrete series parameter for $G$ if $\langle\lambda, \alpha\rangle \neq 0$ for all $\alpha \in \Phi$ and $\lambda-\rho_{G} \in L_{B}$. Let $\lambda$ be a discrete series parameter for $G$ and let $\varphi \in \mathcal{T}(\Phi)$. When we identify $\underline{g}_{\varphi}$ with a subspace of $\underline{g}$, we have $\underline{b}_{\varphi}=\underline{b}$. Thus $\lambda$ is a potential candidate for discrete series parameter of $G_{\varphi}$. Since $\varphi \subset \Phi$, we have $\langle\lambda, \alpha\rangle \neq 0$ for all $\alpha \in \varphi$. Let $\rho_{\varphi}$ denote the half-sum of positive roots in $\varphi$. Then $\lambda-\rho_{\varphi}=\left(\lambda-\rho_{G}\right)+\left(\rho_{G}-\rho_{\varphi}\right)$. Thus $\lambda-\rho_{\varphi} \in L_{B_{\varphi}}=L_{B}$ if and only if $\rho_{G}-\rho_{\varphi} \in L_{B}$. If $\Phi$ is of type $A_{2 n}, n \geq 1$, then there is always $\varphi \in \mathcal{T}(\Phi)$ such that $\rho_{G}-\rho_{\varphi} \notin L_{B}$. However we prove in $\S 5$ that if $\Phi$ contains no irreducible factors of type $A_{2 n}, n \geq 1$, then $\rho_{G}-\rho_{\varphi}$ is in the root lattice of $\Phi$, and hence always is an element of $L_{B}$. As a consequence of this result we have the following theorem.

Theorem 1.1. Suppose that $\Phi$ contains no irreducible factors of type $A_{2 n}, n \geq 1$. Let $\lambda \in i \underline{b}^{*}$ be a discrete series parameter for $G$. Then for every $\varphi \in \mathcal{T}(\Phi), \lambda$ is also a discrete series parameter for $G_{\varphi}$.

Because of Theorem 1.1, we will assume that $\Phi$ contains no irreducible factors of type $A_{2 n}, n \geq 1$. For any discrete series parameter $\lambda$ for $G$, we let $\Theta_{\lambda}$ denote the character of the discrete series representation (actually relative discrete series if $G$ has noncompact center) of $G$ corresponding to $\lambda$. Similarly, we let $\Theta_{\lambda}^{\varphi}$ denote the character of the (relative) discrete series representation of $G_{\varphi}$ corresponding to $\lambda$.

In $\S 6$ we will define (and give an explicit formula for) a positive integer $c(g)$ for each $g \in G^{\prime}$. It depends only on the orbit of $g$ in $G$ and is constant on connected components of Cartan subgroups. We also define signs $\epsilon_{\varphi}^{G}(\lambda)= \pm 1, \varphi \in \mathcal{T}(\Phi)$, corresponding to discrete series parameters of $G$. The main result of this paper is the following character formula.

Theorem 1.2. There is a dense open subset $G^{\prime \prime}$ of $G^{\prime}$ so that

$$
\Theta_{\lambda}(g)=c(g) \sum_{\varphi \in \mathcal{T}(\Phi)} \epsilon_{\varphi}^{G}(\lambda)\left(\operatorname{Lift}_{\varphi}^{G} \Theta_{\lambda}^{\varphi}\right)(g), \quad g \in G^{\prime \prime} .
$$


Since all $\varphi \in \mathcal{T}(\Phi)$ are conjugate via $W(\Phi)$, the complex Lie algebras $\left(\underline{g}_{\varphi}\right)_{\mathbf{C}}$ are all isomorphic for different $\varphi$. However, the real forms $\underline{g}_{\varphi}$ and hence the groups $G_{\varphi}$, can vary with $\varphi$ depending on which roots of $\varphi$ are compact and noncompact. For a fixed $\varphi \in \mathcal{T}(\Phi)$, the orbit mapping $F_{\varphi}$ need not be surjective, and $\operatorname{Lift}_{\varphi}^{G} \Theta_{\lambda}^{\varphi}$ will be zero on any orbit not in the image of $F_{\varphi}$. We need the contributions from all $\varphi \in \mathcal{T}(\Phi)$ in order to recover $\Theta_{\lambda}$ on $G$.

Theorem 1.2 is stated more precisely as Theorem 6.4. Although for simplicity we have only stated it for discrete series characters here, it is also valid for limits of discrete series. It is proven by first directly computing the lifts of the discrete series characters $\Theta_{\lambda}^{\varphi}$ using an explicit description of the sets $X_{\varphi}(g), g \in G^{\prime \prime}$, given in Theorem 4.13. Then the machinery of two-structures and the formula from [ 5 expressing discrete series constants on $G$ in terms of two-structures are used to show that the sum of the lifts is $\Theta_{\lambda}$.

Suppose that $\Phi$ contains an irreducible factor of type $A_{2 n}, n \geq 1$. Then there is an invariant neighborhood $\Omega$ of the identity in $G$ with the following properties. Let $\varphi \in \mathcal{T}(\Phi)$ and let $\Omega_{\varphi}^{\prime}$ denote the union of all good orbits in $G_{\varphi}$ which map into $\Omega \cap G^{\prime}$ via the orbit correspondence $F_{\varphi}$. Then for any discrete series parameter $\lambda$ of $G$ we can define a class function $\Theta_{\lambda}^{\varphi}$ on $\Omega_{\varphi}^{\prime}$ which is a discrete series character of a two-fold cover of $G_{\varphi}$. Further, we can define $\operatorname{Lift}_{\varphi}^{G} \Theta_{\lambda}^{\varphi}$ in $\Omega \cap G^{\prime}$, and the formula of Theorem 1.2 is valid for $g \in \Omega \cap G^{\prime \prime}$.

In the case that $G$ is linear, the work of Shelstad [10, 11, 12] on endoscopy gives a formula for discrete series characters in terms of the lifts of stable discrete series characters on endoscopic groups. The formula in Theorem 1.2 is independent of this theory. The group $G$ is not required to be linear, two-structure groups are not necessarily endoscopic groups, and no stable discrete series characters are used. In the linear case, a lifting formula similar to Theorem 1.2 expressing stable discrete series characters on $G$ in terms of stable discrete series characters on two-structure groups is given in [6]. For an expository account of the results on discrete series characters in [5, 6] and this paper, see [7].

The organization of the paper is as follows. In $\S 2$ we review the definition of and well-known results about the Cartan subgroups $H_{S}, S \in \mathcal{S O} O(\Phi)$. The main result is Theorem 2.5 which characterizes which elements $h \in H_{S}^{1}, h^{\prime} \in H_{S^{\prime}}^{1}, S, S^{\prime} \in \mathcal{S} O(\Phi)$, are conjugate. In $\S 3$ we review discrete series character formulas for $G$, and rewrite them in a form convenient for lifting. The main theorem from [5] expanding discrete series constants in terms of two-structures is stated as Theorem 3.4. The definition of the groups $G_{\varphi}$ and the orbit correspondence are in $\S 4$. The main results are Lemma 4.8 which shows that the orbit map is well-defined, and Theorem 4.13 which gives an explicit description of the sets $X_{\varphi}(g), g \in G^{\prime \prime}$, occurring in the definition of lifting. In $\S 5$ we review the theory of two-structures and prove results about two-structures which will be needed. In particular, we prove in Theorem 5.5 that $\rho_{G}-\rho_{\varphi}$ is in the root lattice of $\Phi$ in the case that $\Phi$ contains no irreducible factors of type $A_{2 n}$. In $\S 6$ we restate and prove Theorem 1.2 as Theorem 6.4, modulo a technical result about two-structures, Lemma 6.8. This technical lemma is proven in $\S 7$.

\section{Regular Semisimple Orbits in $G$}

In this section we restate well-known results about regular semisimple orbits in $G$ in a form that is convenient for discrete series character formulas and the orbit 
correspondence. The main result is Theorem 2.5. Proofs of routine lemmas are omitted.

Let $G$ be a connected reductive Lie group. Given any subgroup $H$ of $G$ we will use the corresponding lower case underlined letter $\underline{h}$ for the real Lie algebra of $H$, and $\underline{h}_{\mathbf{C}}$ for its complexification. For any root system $\Phi$, we let $W(\Phi)$ denote the Weyl group of $\Phi$.

Let $\theta$ be a Cartan involution of $G$ as in [13] and let $K$ be the fixed point set of $\theta$. In the case that $G$ has compact center, $K$ is a maximal compact subgroup of $G$. In general, $K$ contains the center $Z_{G}$ of $G$ and is compact modulo $Z_{G}$. Now $G$ has relative discrete series representations just in case $\operatorname{rank} G=\operatorname{rank} K$ so that $G$ has a Cartan subgroup $B \subset K$. We will assume that this is the case. Then $B$ is a relatively compact Cartan subgroup of $G$, that is $B$ is compact modulo $Z_{G}$, and is unique up to conjugacy. Let $\Phi=\Phi\left(\underline{g}_{\mathbf{C}}, \underline{b}_{\mathbf{C}}\right)$ denote the roots of $\underline{b}_{\mathbf{C}}$ in $\underline{g}_{\mathbf{C}}$ and $\Phi_{K}=\Phi\left(\underline{k}_{\mathbf{C}}, \underline{b}_{\mathbf{C}}\right)$ denote the roots of $\underline{b}_{\mathbf{C}}$ in $\underline{k}_{\mathbf{C}}$. Roots in $\Phi_{K}$ are called compact roots.

Since $G$ is reductive, $\underline{g}=\underline{z}+\underline{g}_{s}$ where $\underline{z}$ is the center of $\underline{g}$ and $\underline{g}_{s}$ is semisimple. We also have $\underline{b}=\underline{z}+\underline{b}_{s}$ where $\underline{b}_{s}=\underline{b} \cap \underline{g}_{s}$. For each $\alpha \in \Phi$ we let $\left(\underline{g}_{\alpha}\right)_{\mathbf{C}}$ denote the root space corresponding to $\alpha$ in $\left(\underline{g}_{s}\right)_{\mathbf{C}}$ and write $H_{\alpha}^{*}$ for the element of $\left(\underline{b}_{s}\right)_{\mathbf{C}}$ satisfying

$$
\beta\left(H_{\alpha}^{*}\right)=2\langle\beta, \alpha\rangle /\langle\alpha, \alpha\rangle, \forall \beta \in \Phi .
$$

For each noncompact $\alpha \in \Phi$ fix a Cayley transform $c_{\alpha}$. Recall from page 418 of [9] that we can choose $X_{\alpha} \in\left(\underline{g}_{\alpha}\right)_{\mathbf{C}}, Y_{\alpha} \in\left(\underline{g}_{-\alpha}\right)_{\mathbf{C}}$ so that

$$
c_{\alpha}=\operatorname{Ad} \exp (\pi / 4)\left(Y_{\alpha}-X_{\alpha}\right) \text {. }
$$

Let $\mathcal{S} O(\Phi)$ denote the set of all subsets of $\Phi$ consisting of strongly orthogonal noncompact roots. That is, $S \in \mathcal{S} O(\Phi)$ if $S \subset \Phi \backslash \Phi_{K}$ and for any $\alpha, \beta \in S$ with $\alpha \neq \beta,\langle\alpha, \beta\rangle=0$ and $\alpha \pm \beta \notin \Phi$. Each $S \in \mathcal{S} O(\Phi)$ corresponds to a Cartan subgroup $H_{S}$ of $G$ as follows. Define

$$
\underline{t}_{S}=\{H \in \underline{b}: \alpha(H)=0 \forall \alpha \in S\}, \quad \underline{b}_{S}=\sum_{\alpha \in S} i \mathbf{R} H_{\alpha}^{*} .
$$

Let $c_{S}=\prod_{\alpha \in S} c_{\alpha}$. Then $H_{S}$ is the Cartan subgroup of $G$ with Lie algebra

$$
\underline{h}_{S}=\underline{t}_{S} \oplus \underline{a}_{S}
$$

where

$$
\underline{a}_{S}=c_{S}\left(i \underline{b}_{S}\right)=\sum_{\alpha \in S} \mathbf{R}\left(X_{\alpha}+Y_{\alpha}\right) .
$$

It satisfies $\left(\underline{h}_{S}\right)_{\mathbf{C}}=c_{S}\left(\underline{b}_{\mathbf{C}}\right)$ since $c_{S}$ is the identity on $\underline{t}_{S}$. Define $T_{S}=H_{S} \cap K$ and $A_{S}=\exp \left(\underline{a}_{S}\right)$. Then $H_{S}=T_{S} A_{S}$. Note that $T_{S}$ need not be connected. The identity component $T_{S}^{0}$ of $T_{S}$ is contained in $B$, but in general not every connected component of $T_{S}$ will lie in $B$. Write $T_{S}^{1}=T_{S} \cap B, H_{S}^{1}=T_{S}^{1} A_{S}$. While $T_{S}$ need not be abelian when $G$ is not linear, $T_{S}^{1}$ is always abelian, since $B$ is. Thus $H_{S}^{1}$ is always abelian. When we write $h=t a \in H_{S}^{1}$ we always mean that $t \in T_{S}^{1}$ and $a \in A_{S}$.

Fix $t \in T_{S}^{1}$. Since $t T_{S}^{0} \subset B$ and every $\alpha \in \Phi$ determines a character $e^{\alpha}$ of $B$, we can define

$$
\Phi_{t, S}=\left\{\alpha \in \Phi: e^{\alpha}\left(t t_{0}\right)=1 \forall t_{0} \in T_{S}^{0}\right\}
$$


When $S$ is fixed we will also write $\Phi_{t}=\Phi_{t, S}, t \in T_{S}^{1}$.

Lemma 2.1. Fix $S \in \mathcal{S} O(\Phi)$. Then we have the following.

(i) $T_{S}^{1}=\left(T_{S} \cap B_{S}\right) T_{S}^{0}$ where $B_{S}=\exp \left(\underline{b}_{S}\right)$.

(ii) $T_{S} \cap B_{S}$ consists of all elements of the form $\exp \left(\pi i \sum_{\alpha \in S} n_{\alpha} H_{\alpha}^{*}\right)$ where $n_{\alpha} \in \mathbf{Z}$ for all $\alpha \in S$.

(iii) $T_{S}^{1}=\left\{b \in B: e^{\alpha}(b)=1 \forall \alpha \in S\right\}$.

(iv) For all $t \in T_{S}^{1}, S \subset \Phi_{t, S}$ and spans $\Phi_{t, S}$.

(v) For $b \in T_{S}^{1}, e^{\beta}(b)=e^{c_{S} \beta}(b)$ for all $\beta \in \Phi$.

For $S, S^{\prime} \in \mathcal{S} O(\Phi)$, we define $S \equiv S^{\prime}$ if $\underline{t}_{S}=\underline{t}_{S^{\prime}}$. Let $v \in W\left(\Phi_{K}\right), H \in \underline{b}$, and $b=\exp (H) \in B$. Then we write $v b=\exp (v H)$. This is well-defined and gives an identification of $W\left(\Phi_{K}\right)$ with $W(G, B)=N_{G}(B) / B$. Let $G^{\prime}$ denote the set of regular semisimple elements of $G$.

Lemma 2.2. (i) Let $S, S^{\prime} \in \mathcal{S} O(\Phi)$. Then $H_{S}$ is conjugate to $H_{S^{\prime}}$ in $G$ if and only if there is $v \in W\left(\Phi_{K}\right)$ such that $S^{\prime} \equiv v S$.

(ii) Given any $x \in G^{\prime}$ there are $S \in \mathcal{S O}(\Phi)$ and $h \in H_{S}^{1}$ such that $\mathcal{O}_{G}(x)=$ $\mathcal{O}_{G}(h)$.

Lemma 2.3. Let $S, S^{\prime} \in \mathcal{S} O(\Phi)$ with Cayley transforms $c_{S}, c_{S^{\prime}}$ respectively. Let $h=t a \in H_{S}^{1} \cap G^{\prime}, h^{\prime}=t^{\prime} a^{\prime} \in H_{S^{\prime}}^{1} \cap G^{\prime}$.

(i) Suppose there is $x \in G$ such that $x h x^{-1}=h^{\prime}$. Then there are $v \in W\left(\Phi_{K}\right), s \in$ $W\left(\Phi_{t}\right)$, such that $t^{\prime}=v t, S^{\prime} \equiv v S, \log a^{\prime}=c_{S^{\prime}} v s c_{S}^{-1} \log a$, and $A d(x) H=$ $c_{S^{\prime}} v s c_{S}^{-1} H$ for all $H \in \underline{h}_{S}$.

(ii) Suppose that there are $v \in W\left(\Phi_{K}\right), s \in W\left(\Phi_{t}\right)$, such that $t^{\prime}=v t, S^{\prime} \equiv v S$, and $\log a^{\prime}=c_{S^{\prime}} v s c_{S}^{-1} \log a$. Then there is $x \in G$ such that $h^{\prime}=x h x^{-1}$ and $A d(x) H=c_{S^{\prime}} v s c_{S}^{-1} H$ for all $H \in \underline{h}_{S}$.

For $S, S^{\prime} \in \mathcal{S} O(\Phi), t \in T_{S}^{1}$, we will write $S^{\prime} \equiv_{t} S$ if $S^{\prime} \equiv S$ and $t \in T_{S^{\prime}}^{1}$. In this case we have $t T_{S}^{0}=t T_{S^{\prime}}^{0}$ so that $\Phi_{t, S}=\Phi_{t, S^{\prime}}$. By Lemma $2.1, S^{\prime} \equiv_{t} S$ if and only if $S^{\prime} \subset \Phi_{t, S}$ and spans $\Phi_{t, S}$.

Lemma 2.4. Fix $h=t a \in H_{S}^{1}, v \in W\left(\Phi_{K}\right), s \in W\left(\Phi_{t}\right)$, and $S^{\prime} \in \mathcal{S} O(\Phi)$ such that $v^{-1} S^{\prime} \equiv_{t} S$. Then there is an element $h^{\prime}=t^{\prime} a^{\prime} \in H_{S^{\prime}}^{1}$ given by

$$
t^{\prime}=v t, \quad \log a^{\prime}=c_{S^{\prime}} v s c_{S}^{-1} \log a .
$$

For $S \in \mathcal{S} O(\Phi), h=t a \in H_{S}^{1}$, we will let $W_{0}(h)$ denote the set of all $u \in$ $W\left(\Phi_{K}\right) \cap W\left(\Phi_{t}\right)$ such that $u t=t$. Then for $(v, s),\left(v^{\prime}, s^{\prime}\right) \in W\left(\Phi_{K}\right) \times W\left(\Phi_{t}\right)$, we will say $(v, s) \sim\left(v^{\prime}, s^{\prime}\right)$ if there is $u \in W_{0}(h)$ such that $v^{\prime}=v u, s^{\prime}=u^{-1} s$. This gives an equivalence relation on $W\left(\Phi_{K}\right) \times W\left(\Phi_{t}\right)$ and we write $[v, s]$ for the equivalence class containing $(v, s)$ and $W\left(\Phi_{K}, h\right)$ for the set of equivalence classes. We write $w=1$ for the equivalence class $[1,1]$.

Remark 2.1. In Lemma 2.7 of [7] we prove that $W_{0}(h)=W\left(\Phi_{K}\right) \cap W\left(\Phi_{t}\right)$ if and only if $h$ is central in $H_{S}$. Of course if all Cartan subgroups of $G$ are abelian, as is the case when $G$ is linear, then this implies that $W_{0}(h)=W\left(\Phi_{K}\right) \cap W\left(\Phi_{t}\right)$ always. Thus in this case we can identify $W\left(\Phi_{K}, h\right)$ with the set of all $w \in W(\Phi)$ such that there are $v \in W\left(\Phi_{K}\right), s \in W\left(\Phi_{t}\right)$ such that $w=v s$. However when $W_{0}(h)$ is a proper subgroup of $W\left(\Phi_{K}\right) \cap W\left(\Phi_{t}\right), W\left(\Phi_{K}, h\right)$ cannot be identified with a subset of $W(\Phi)$. 
Let $v \in W\left(\Phi_{K}\right)$ and $S^{\prime} \in \mathcal{S} O(\Phi)$ with $v^{-1} S^{\prime} \equiv_{t} S$. Then for any $u \in W_{0}(h)$, we also have $(v u)^{-1} S^{\prime} \equiv_{t} S$ since $t=u^{-1} t \in u^{-1} T_{v^{-1} S^{\prime}}^{1}=T_{u^{-1} v^{-1} S^{\prime}}^{1}$. Thus we can write $\Lambda(h)$ for the set of all pairs $\left(w, S^{\prime}\right)$ such that $w=[v, s] \in W\left(\Phi_{K}, h\right), S^{\prime} \in$ $\mathcal{S} O(\Phi)$, and $v^{-1} S^{\prime} \equiv_{t} S$. Note that for any $w=[v, s] \in W\left(\Phi_{K}, h\right),(w, v S) \in \Lambda(h)$. However there may be other $S^{\prime} \in \mathcal{S} O(\Phi)$ with $\left(w, S^{\prime}\right) \in \Lambda(h)$.

Let $\left(w, S^{\prime}\right) \in \Lambda(h)$, and let $(v, s) \in W\left(\Phi_{K}\right) \times W\left(\Phi_{t}\right)$ such that $w=[v, s]$. We will write $h^{\prime}=\left(w, S^{\prime}\right) \cdot h$ for the element $h^{\prime}=t^{\prime} a^{\prime} \in H_{S^{\prime}}^{1}$ given as in Lemma 2.4 by

$$
t^{\prime}=v t, \quad \log a^{\prime}=c_{S^{\prime}} v s c_{S}^{-1} \log a .
$$

This definition clearly does not depend on the representative $(v, s)$ for $w$.

Note that $\Lambda(h)$ depends only on the connected component $h H_{S}^{0}$ of $h$ in $H_{S}^{1}$. In fact, as a consequence of Lemma 2.3 (ii), we see that for $\left(w, S^{\prime}\right) \in \Lambda(h)$ there is $x \in G$ such that $\left(w, S^{\prime}\right) \cdot h_{1}=x h_{1} x^{-1}$ for all $h_{1} \in h H_{S}^{0}$.

For any $x \in G$, we let $\mathcal{O}_{G}(x)=\left\{g x g^{-1}: g \in G\right\}$ denote the orbit of $x$ in $G$. Using the above notation, we can rewrite Lemma 2.3 as follows.

Theorem 2.5. Let $S \in \mathcal{S} O(\Phi), h \in H_{S}^{1} \cap G^{\prime}$. Then

$$
\mathcal{O}_{G}(h) \cap\left(\bigcup_{S^{\prime} \in \mathcal{S} O(\Phi)} H_{S^{\prime}}^{1}\right)=\left\{\left(w, S^{\prime}\right) \cdot h:\left(w, S^{\prime}\right) \in \Lambda(h)\right\} .
$$

Lemma 2.6. Let $S \in \mathcal{S} O(\Phi), h=t a \in H_{S}^{1} \cap G^{\prime}$. Fix $\left(w, S^{\prime}\right) \in \Lambda(h)$, and let $h^{\prime}=t^{\prime} a^{\prime}=\left(w, S^{\prime}\right) \cdot h$. Let $(v, s) \in W\left(\Phi_{K}\right) \times W\left(\Phi_{t}\right)$ such that $w=[v, s]$. Then $w^{-1}=\left[v^{-1}, v s^{-1} v^{-1}\right] \in W\left(\Phi_{K}, h^{\prime}\right),\left(w^{-1}, S\right) \in \Lambda\left(h^{\prime}\right)$ and $h=\left(w^{-1}, S\right) \cdot h^{\prime}$. Further, suppose $\left(w^{\prime}, S^{\prime \prime}\right) \in \Lambda\left(h^{\prime}\right)$ and let $\left(v^{\prime}, s^{\prime}\right) \in W\left(\Phi_{K}\right) \times W\left(\Phi_{t^{\prime}}\right)$ such that $w^{\prime}=\left[v^{\prime}, s^{\prime}\right]$. Then $w^{\prime} w=\left[v^{\prime} v, v^{-1} s^{\prime} v s\right] \in W\left(\Phi_{K}, h\right),\left(w^{\prime} w, S^{\prime \prime}\right) \in \Lambda(h)$ and $\left(w^{\prime}, S^{\prime \prime}\right) \cdot h^{\prime}=\left(w^{\prime} w, S^{\prime \prime}\right) \cdot h$.

In order to prove the lifting theorem we will need to restrict to a dense open subset of $H_{S}^{1} \cap G^{\prime}$. Define

$$
H_{S}^{\prime \prime}=\left\{h \in H_{S}^{1} \cap G^{\prime}: \text { if }(w, S) \in \Lambda(h) \text { and }(w, S) \cdot h=h, \text { then } w=1\right\} .
$$

Remark 2.2. $H_{S}^{\prime \prime}$ can be a proper subset of $H_{S}^{1} \cap G^{\prime}$. For example, suppose that $G$ is a simple group of adjoint type with $\Phi=\{ \pm \alpha\}$ of type $A_{1}$. Then $B=H_{\emptyset}$ consists of all elements of the form $b(s)=\exp \left(i s H_{\alpha}^{*}\right)$ and $b(s)=1$ just in case $s \in \pi \mathbf{Z}$. Further, $W\left(\Phi_{K}, b\right)=W\left(\Phi_{K}\right)$ and $\Lambda(b)=\left\{(w, \emptyset): w \in W\left(\Phi_{K}\right)\right\}$ for all $b \in B$. Suppose that $G$ is noncompact. Then $\Phi_{K}=\emptyset$, so that $B^{\prime \prime}=B \cap G^{\prime}$. However, if $G$ is compact, then $\Phi_{K}=\Phi$ and the reflection $s_{\alpha} \in W\left(\Phi_{K}\right)$. Now $b=b(\pi / 2)$ is regular since $\exp (\alpha)(b)=-1$, but $\left(s_{\alpha}, \emptyset\right) \cdot b=b(-\pi / 2)=b$. Thus $b \notin B^{\prime \prime}$.

Lemma 2.7. Let $S \in \mathcal{S} O(\Phi)$. Then $H_{S}^{\prime \prime}$ is a dense open subset of $H_{S}^{1} \cap G^{\prime}$. It is the set of all $h \in H_{S}^{1}$ such that $C_{G}(h) \subset H_{S}$.

Proof. The first statement is routine. The second follows easily from Lemma 2.3.

Remark 2.3. Suppose that $G$ has abelian Cartan subgroups. Then $H_{S} \subset C_{G}(h)$ for all $h \in H_{S}^{1}$. Thus in this case $h \in H_{S}^{\prime \prime}$ if and only if $C_{G}(h)=H_{S}$. When $G$ is the set of real points of a linear algebraic group $\mathbf{G}$, an element $g \in G$ is called strongly regular if its centralizer in $\mathbf{G}$ is a (connected) torus. Suppose that $h \in H_{S}^{1}$ is strongly regular. Then its centralizer in $G$ is the set of real points of a torus of G, and hence must be equal to $H_{S}$. Thus $h \in H_{S}^{\prime \prime}$. 


\section{Discrete Series on $G$}

In this section we review discrete series character formulas and rewrite them using the notation of $\S 2$. In the case that $G$ has noncompact center these are really relative discrete series, but this will play no role in the arguments, so we will just use the term discrete series. For more details see [5, 7, 9].

Fix a choice $\Phi^{+}$of positive roots for $\Phi$, and define

$$
\rho_{G}=\rho\left(\Phi^{+}\right)=\frac{1}{2} \sum_{\alpha \in \Phi^{+}} \alpha
$$

Let $L_{B}$ denote the set of all $\tau \in E=i \underline{b}^{*}$ such that $\exp (\tau)$ is well-defined on $B$. Recall that $\lambda \in i \underline{b}^{*}$ is called a discrete series parameter for $G$ if

$$
\lambda-\rho_{G} \in L_{B} \text { and }\langle\lambda, \alpha\rangle \neq 0 \forall \alpha \in \Phi .
$$

Further, $\lambda \in i \underline{b}^{*}$ is called a limit of discrete series parameter for $G$ if it is not a discrete series parameter, but $\lambda-\rho_{G} \in L_{B}$. Let

$$
E^{\prime}(\Phi)=\{\tau \in E:\langle\tau, \alpha\rangle \neq 0 \forall \alpha \in \Phi\} .
$$

For any connected component $E^{*}$ of $E^{\prime}(\Phi)$, define

$$
\epsilon_{G}\left(\Phi^{+}: E^{*}\right)=\epsilon_{G}\left(E^{*}\right)=\operatorname{sign} \prod_{\alpha \in \Phi^{+}}\langle\alpha, \tau\rangle, \quad \tau \in E^{*} .
$$

Fix a discrete series or limit of discrete series parameter $\lambda$ of $G$. If $\lambda$ is a discrete series parameter, let $E^{*}=E^{*}(\lambda)$ denote the connected component of $E^{\prime}(\Phi)$ containing $\lambda$. If $\lambda$ is a limit of discrete series parameter, let $E^{*}=E^{*}(\lambda)$ be a fixed connected component of $E^{\prime}(\Phi)$ such that $\lambda$ is in the closure of $E^{*}(\lambda)$.

Write

$$
\Delta_{G}^{\prime}\left(\Phi^{+}: b\right)=\Delta_{G}^{\prime}(b)=\prod_{\alpha \in \Phi^{+}}\left(1-e^{-\alpha}(b)\right), \quad b \in B
$$

and define

$$
q_{G}=\frac{1}{2} \operatorname{dim}(G / K)
$$

Then as in [1, 2, 8], when $\lambda$ is a discrete series parameter there is a unique (relative) discrete series representation of $G$ with character $\Theta_{\lambda}$ given on regular elements $b \in B$ by

$$
\Theta_{\lambda}(b)=\epsilon_{G}\left(E^{*}\right)(-1)^{q_{G}} \Delta_{G}^{\prime}(b)^{-1} \sum_{v \in W\left(\Phi_{K}\right)} \operatorname{det} v e^{v \lambda-\rho_{G}}(b) .
$$

Note that although $\epsilon_{G}\left(E^{*}\right), \Delta_{G}^{\prime}(b)$, and $\rho_{G}$ all depend on the choice $\Phi^{+}$of positive roots, the character $\Theta_{\lambda}$ is independent of this choice.

Now let $S \in \mathcal{S} O(\Phi)$ with corresponding Cayley transform $c_{S}$ and Cartan subgroup $H_{S}$. Then $c_{S} \Phi^{+}$gives a choice of positive roots for $\Phi\left(\underline{g}_{\mathbf{C}},\left(\underline{h}_{S}\right)_{\mathbf{C}}\right)$. For $h \in H_{S}$, define

$$
\Delta_{G}^{\prime}\left(\Phi^{+}: h\right)=\Delta_{G}^{\prime}(h)=\prod_{\alpha \in c_{S} \Phi^{+}}\left(1-e^{-\alpha}(h)\right) .
$$

For $h=t a \in H_{S}^{1}$, let

$$
\Phi_{R}^{+}(h)=\left\{\alpha \in \Phi_{t}: \alpha\left(c_{S}^{-1} \log a\right)>0\right\} .
$$


Define $\Phi_{K, t}=\Phi_{K} \cap \Phi_{t}$. Then $W\left(\Phi_{K, t}\right) \subset W_{0}(h)=\left\{u \in W\left(\Phi_{K}\right) \cap W\left(\Phi_{t}\right): u t=t\right\}$. It is possible that $W\left(\Phi_{K, t}\right)$ is a proper subgroup of $W_{0}(h)$. Define

$$
c(K, h)=\left[W_{0}(h) / W\left(\Phi_{K, t}\right)\right] .
$$

Lemma 3.1. For all $h=t a \in H_{S}^{1} \cap G^{\prime}$ we have

$$
\begin{gathered}
\epsilon_{G}\left(E^{*}\right)(-1)^{q_{G}} \Delta_{G}^{\prime}(h) \Theta_{\lambda}(h)=c(K, h) \sum_{v \in W_{0}(h) \backslash W\left(\Phi_{K}\right)} \operatorname{det} v e^{v \lambda-\rho_{G}}(t) \\
\times \sum_{s \in W\left(\Phi_{t}\right)} \operatorname{det} s c\left(s: v E^{*}: \Phi_{R}^{+}(h)\right) \exp \left(\left(s v \lambda-\rho_{G}\right)\left(c_{S}^{-1} \log a\right)\right) .
\end{gathered}
$$

Here the constants $c\left(s: v E^{*}: \Phi_{R}^{+}(h)\right)$ are defined as in [5].

Proof. We will use formula (2.6) from [5], which is a restatement of

Harish-Chandra's formulas from $\S 23$ of [1]. In [5] the results are only stated for the semisimple finite center case, but as in 8 , the formulas are also valid for the general case. Note that in [5] $\Theta_{\lambda}$ was defined without the terms $\epsilon_{G}\left(E^{*}\right)(-1)^{q_{G}}$ and was only a character up to sign. The root system $\Phi$ in [5] is our $\Phi_{t}$. Thus in our notation we can rewrite formula (2.6) of [5] as

$$
\begin{gathered}
\epsilon_{G}\left(E^{*}\right)(-1)^{q_{G}} \Delta_{G}^{\prime}(h) \Theta_{\lambda}(h)=\sum_{v \in W\left(\Phi_{K, t}\right) \backslash W\left(\Phi_{K}\right)} \operatorname{det} v e^{v \lambda-\rho_{G}}(t) \\
\times \sum_{s \in W\left(\Phi_{t}\right)} \operatorname{det} s c\left(s: v E^{*}: \Phi_{R}^{+}(h)\right) \exp \left(\left(s v \lambda-\rho_{G}\right)\left(c_{S}^{-1} \log a\right)\right) \\
=\sum_{v \in W_{0}(h) \backslash W\left(\Phi_{K}\right)} \sum_{u \in W\left(\Phi_{K, t}\right) \backslash W_{0}(h)} \operatorname{det}(u v) e^{u v \lambda-\rho_{G}}(t) \\
\times \sum_{s \in W\left(\Phi_{t}\right)} \operatorname{det} s c\left(s: u v E^{*}: \Phi_{R}^{+}(h)\right) \exp \left(\left(\operatorname{suv} \lambda-\rho_{G}\right)\left(c_{S}^{-1} \log a\right)\right) \\
=\sum_{v \in W_{0}(h) \backslash W\left(\Phi_{K}\right) \quad u \in W\left(\Phi_{K, t}\right) \backslash W_{0}(h)} \operatorname{det} v e^{u v \lambda-\rho_{G}}(t) \\
\times \sum_{s \in W\left(\Phi_{t}\right)} \operatorname{det} s c\left(s u^{-1}: u v E^{*}: \Phi_{R}^{+}(h)\right) \exp \left(\left(s v \lambda-\rho_{G}\right)\left(c_{S}^{-1} \log a\right)\right) .
\end{gathered}
$$

Fix $u \in W\left(\Phi_{K}\right) \cap W\left(\Phi_{t}\right)$ and define $\psi: \Phi_{t} \rightarrow \Phi_{t}$ by $\psi(\alpha)=u \alpha$. Since $u \Phi_{K}=\Phi_{K}$ and $u \Phi_{t}=\Phi_{t}, u \Phi_{K, t}=\Phi_{K, t}$. Further, for $s \in W\left(\Phi_{t}\right), \psi s \psi^{-1}=u s u^{-1}$. Thus using (3.1), (3.6) of [5] we have

$$
c\left(s: v E^{*}: \Phi_{R}^{+}(h)\right)=c\left(u s u^{-1}: u v E^{*}: u \Phi_{R}^{+}(h)\right)=c\left(s u^{-1}: u v E^{*}: \Phi_{R}^{+}(h)\right) .
$$

Further,

$$
e^{u v \lambda-\rho_{G}}(t)=e^{v \lambda-u^{-1} \rho_{G}}\left(u^{-1} t\right)=e^{v \lambda-\rho_{G}}\left(u^{-1} t\right) e^{u \rho_{G}-\rho_{G}}(t) .
$$

But $\rho_{G}$ is a weight for $\Phi$ and hence $\Phi_{t}$, so that $u \rho_{G}-\rho_{G}$ is in the root lattice of $\Phi_{t}$. Thus $e^{u \rho_{G}-\rho_{G}}(t)=1$. Now if $u \in W_{0}(h) \subset W\left(\Phi_{K}\right) \cap W\left(\Phi_{t}\right)$, we have $u^{-1} t=t$ so that

$$
e^{u v \lambda-\rho_{G}}(t)=e^{v \lambda-\rho_{G}}(t) .
$$


When $\lambda$ is a limit of discrete series parameter, we define $\Theta_{\lambda}$ using the formula in Lemma 3.1, noting that it depends on the fixed choice of $E^{*}=E^{*}(\lambda)$. We can simplify the notation of Lemma 3.1 as follows. Let $\tau \in L_{B}$. Then for any $h=t a \in H_{S}^{1}$ we write

$$
\xi_{\tau}(h)=e^{\tau}(t) \exp \left(\tau\left(c_{S}^{-1} \log a\right)\right) .
$$

Let $w=[v, s] \in W\left(\Phi_{K}, h\right)$, and pick $S^{\prime} \in \mathcal{S} O(\Phi)$ such that $\left(w, S^{\prime}\right) \in \Lambda(h)$. (Recall that such an $S^{\prime}$ always exists since we can always take $S^{\prime}=v S$.) Then we define

$$
\begin{gathered}
\xi_{\tau}(w \cdot h)=\xi_{\tau}\left(\left(w, S^{\prime}\right) \cdot h\right), \Delta_{G}^{\prime}(w \cdot h)=\Delta_{G}^{\prime}\left(\left(w, S^{\prime}\right) \cdot h\right), \\
\Phi_{R}^{+}(w \cdot h)=\Phi_{R}^{+}\left(\left(w, S^{\prime}\right) \cdot h\right) .
\end{gathered}
$$

It is easy to check that $\xi_{\tau}\left(\left(w, S^{\prime}\right) \cdot h\right), \Delta_{G}^{\prime}\left(\left(w, S^{\prime}\right) \cdot h\right)$, and $\Phi_{R}^{+}\left(\left(w, S^{\prime}\right) \cdot h\right)$ are independent of the choice of $S^{\prime}$. Thus $\xi_{\tau}(w \cdot h), \Delta_{G}^{\prime}(w \cdot h)$, and $\Phi_{R}^{+}(w \cdot h)$ are welldefined. We also define $\operatorname{det} w=\operatorname{det} v \operatorname{det} s$, and for $\lambda \in i \underline{b}^{*}$, we write $w \lambda=v s \lambda$. These definitions are clearly independent of the choice of representative $(v, s)$.

Lemma 3.2. Let $w=[v, s] \in W\left(\Phi_{K}, h\right), h=t a \in H_{S}^{1}$.

(i) For any $\tau \in L_{B}, \xi_{\tau}(w \cdot h)=e^{v^{-1} \tau}(t) \exp \left(\left(s^{-1} v^{-1} \tau\right)\left(c_{S}^{-1} \log a\right)\right)$.

(ii) For any $\alpha \in \Phi, \xi_{\alpha}(w \cdot h)=\xi_{s^{-1} v^{-1} \alpha}(h)$.

(iii) $\Delta_{G}^{\prime}(w \cdot h)=\operatorname{det} w \xi_{\rho_{G}-w^{-1} \rho_{G}}(h) \Delta_{G}^{\prime}(h)$.

(iv) $\Phi_{R}^{+}(w \cdot h)=w \Phi_{R}^{+}(h)$.

(v) $c\left(s^{-1}: v^{-1} E^{*}: \Phi_{R}^{+}(h)\right)=c\left(1: E^{*}: \Phi_{R}^{+}(w \cdot h)\right)$.

Proof. Parts (i)-(iv) are routine. Using part (iv),

$$
c\left(1: E^{*}: \Phi_{R}^{+}(w \cdot h)\right)=c\left(1: E^{*}: v s \Phi_{R}^{+}(h)\right) .
$$

Now define $\psi: v s \Phi_{t} \rightarrow s \Phi_{t}$ by $\psi(\alpha)=v^{-1} \alpha$. Then $\psi\left(v s \Phi_{t} \cap \Phi_{K}\right)=s \Phi_{t} \cap \Phi_{K}$ so that using (3.1). (3.6) of [5],

$$
c\left(1: E^{*}: v s \Phi_{R}^{+}(h)\right)=c\left(1: v^{-1} E^{*}: s \Phi_{R}^{+}(h)\right)=c\left(s^{-1}: v^{-1} E^{*}: \Phi_{R}^{+}(h)\right) .
$$

Theorem 3.3. Let $h \in H_{S}^{1} \cap G^{\prime}$. Then

$$
\begin{aligned}
\epsilon_{G}\left(E^{*}\right) & (-1)^{q_{G}} \Theta_{\lambda}(h) \\
= & c(K, h) \sum_{w \in W\left(\Phi_{K}, h\right)} \Delta_{G}^{\prime}(w \cdot h)^{-1} \xi_{\lambda-\rho_{G}}(w \cdot h) c\left(1: E^{*}: \Phi_{R}^{+}(w \cdot h)\right) .
\end{aligned}
$$

Proof. Using Lemma 3.1 and the change of variables $s \mapsto s^{-1}, v \mapsto v^{-1}$ we can write

$$
\begin{aligned}
& \epsilon_{G}\left(E^{*}\right)(-1)^{q_{G}} \Theta_{\lambda}(h)=c(K, h) \Delta_{G}^{\prime}(h)^{-1} \sum_{w=[v, s] \in W\left(\Phi_{K}, h\right)} \operatorname{det} w \\
& \quad \times e^{v^{-1} \lambda-\rho_{G}}(t) c\left(s^{-1}: v^{-1} E^{*}: \Phi_{R}^{+}(h)\right) \exp \left(\left(s^{-1} v^{-1} \lambda-\rho_{G}\right)\left(c_{S}^{-1} \log a\right)\right) .
\end{aligned}
$$

Let $w=[v, s] \in W\left(\Phi_{K}, h\right)$. Then by Lemma 3.2(iii) we have

$$
\Delta_{G}^{\prime}(w \cdot h)^{-1}=\Delta_{G}^{\prime}(h)^{-1} \operatorname{det} w \xi_{w^{-1} \rho_{G}-\rho_{G}}(h) .
$$


Since $w^{-1} \rho_{G}-\rho_{G}$ is in the root lattice of $\Phi$, we can use Lemma 3.2(i) and (ii) to write

$$
\begin{aligned}
& \xi_{w^{-1} \rho_{G}-\rho_{G}}(h) \xi_{\lambda-\rho_{G}}(w \cdot h)=\xi_{\lambda-w \rho_{G}}(w \cdot h) \\
& =e^{v^{-1} \lambda-s \rho_{G}}(t) \exp \left(\left(s^{-1} v^{-1} \lambda-\rho_{G}\right)\left(c_{S}^{-1} \log a\right)\right) .
\end{aligned}
$$

But $\rho_{G}-s \rho_{G}$ is in the root lattice of $\Phi_{t}$ so that

$$
e^{v^{-1} \lambda-s \rho_{G}}(t)=e^{v^{-1} \lambda-\rho_{G}}(t)
$$

Combining this with Lemma $3.2(\mathrm{v})$, we have

$$
\begin{aligned}
& \sum_{w \in W\left(\Phi_{K}, h\right)} \Delta_{G}^{\prime}(w \cdot h)^{-1} \xi_{\lambda-\rho_{G}}(w \cdot h) c\left(1: E^{*}: \Phi_{R}^{+}(w \cdot h)\right) \\
& =\Delta_{G}^{\prime}(h)^{-1} \sum_{w=[v, s] \in W\left(\Phi_{K}, h\right)} \operatorname{det} w e^{v^{-1} \lambda-\rho_{G}}(t) \\
& \quad \times c\left(s^{-1}: v^{-1} E^{*}: \Phi_{R}^{+}(h)\right) \exp \left(\left(s^{-1} v^{-1} \lambda-\rho_{G}\right)\left(c_{S}^{-1} \log a\right)\right) .
\end{aligned}
$$

The constants appearing in the discrete series character formula of Theorem 3.3 are of the form $c\left(1: E^{*}: \Phi_{t}^{+}\right)$where $\Phi_{t}$ is a root system spanned by strongly orthogonal noncompact roots, $\Phi_{t}^{+}$is a choice of positive roots for $\Phi_{t}$, and $E^{*}$ is a connected component of $E^{\prime}(\Phi)$. These constants satisfy the following identity. See [5] for details.

Let $\mathcal{T}\left(\Phi_{t}\right)$ denote the set of all two-structures for $\Phi_{t}$. We will say $\psi \in \mathcal{T}\left(\Phi_{t}\right)$ is of noncompact type if $\psi$ is spanned by a collection of strongly orthogonal noncompact roots of $\Phi_{t}$, that is there is $S^{\prime} \in \mathcal{S O}(\Phi)$ such that $S^{\prime} \subset \psi$ and spans $\psi$. This is equivalent to the condition that all long roots of $\psi$ are noncompact in $\Phi_{t}$, where by a long root of $\psi$ we mean any root in an $A_{1}$ factor and any long root in a $B_{2} \simeq C_{2}$ factor. Write $\mathcal{T}_{n}\left(\Phi_{t}\right)$ for the two-structures of noncompact type for $\Phi_{t}$.

The following theorem is proven in [5]. It contains signs $\epsilon\left(\psi: \Phi_{t}^{+}\right)= \pm 1$ corresponding to a choice of positive roots $\Phi_{t}^{+}$for $\Phi_{t}$ and $\psi \in \mathcal{T}\left(\Phi_{t}\right)$. The definition of these signs is given in (5.1). It also contains a constant $c\left(\Phi_{t}\right)$ which is defined as follows. For any $\psi \in \mathcal{T}\left(\Phi_{t}\right), L\left(\Phi_{t}\right) \subset L(\psi)$ where $L\left(\Phi_{t}\right), L(\psi)$ denote the weight lattices of $\Phi_{t}$ and $\psi$ respectively. Let

$$
c\left(\Phi_{t}\right)=\left[L(\psi): L\left(\Phi_{t}\right)\right]
$$

It is independent of the choice of $\psi$ since all $\psi \in \mathcal{T}\left(\Phi_{t}\right)$ are conjugate via $W\left(\Phi_{t}\right)$. Its values for $\Phi_{t}$ irreducible are given by the following table, which also gives the type of $\psi$ in each case.

\begin{tabular}{|c|c|c|c|c|c|c|c|c|c|}
\hline$\Phi_{t}$ & $B_{2 n}$ & $B_{2 n+1}$ & $C_{2 n}$ & $C_{2 n+1}$ & $D_{2 n}$ & $E_{7}$ & $E_{8}$ & $F_{4}$ & $G_{2}$ \\
\hline$\psi$ & $B_{2}^{n}$ & $B_{2}^{n} \times B_{1}$ & $C_{2}^{n}$ & $C_{2}^{n} \times C_{1}$ & $A_{1}^{2 n}$ & $A_{1}^{7}$ & $A_{1}^{8}$ & $B_{2}^{2}$ & $A_{1}^{2}$ \\
\hline$c\left(\Phi_{t}\right)$ & $2^{n-1}$ & $2^{n}$ & 1 & 1 & $2^{n-1}$ & $2^{3}$ & $2^{4}$ & 2 & 2 \\
\hline
\end{tabular}

Theorem 3.4. Let $E^{*}$ be any connected component of $E^{\prime}(\Phi)$ and let $\Phi_{t}^{+}$be any choice of positive roots for $\Phi_{t}$. Then

$$
c\left(1: E^{*}: \Phi_{t}^{+}\right)=c\left(\Phi_{t}\right) \sum_{\psi \in \mathcal{T}_{n}\left(\Phi_{t}\right)} \epsilon\left(\psi: \Phi_{t}^{+}\right) c\left(1: E^{*}: \psi \cap \Phi_{t}^{+}\right) .
$$




\section{Definition of $G_{\varphi}$ And the orbit CORRESPOndence}

Let $\Phi$ be any root system. Then a root subsystem $\varphi \subset \Phi$ is called a two-structure for $\Phi$ if it satisfies the following two properties.

(i) Every irreducible factor of $\varphi$ is of type $A_{1}$ or $B_{2} \simeq C_{2}$.

(ii) Let $\varphi^{+}$be any choice of positive roots for $\varphi$. Then if $w \in W(\Phi)$ with $w \varphi^{+}=$ $\varphi^{+}$we have $\operatorname{det} w=1$.

Let $\mathcal{T}(\Phi)$ denote the set of all two-structures for $\Phi$.

Note that $\varphi \in \mathcal{T}(\Phi)$ is a root subsystem of $\Phi$, that is a subset of $\Phi$ which is closed under its own reflections. It is not necessarily a subroot system. That is, there may be $\alpha, \beta \in \varphi$ such that $\alpha+\beta \in \Phi$, but $\alpha+\beta \notin \varphi$. However, let $\varphi_{0}$ be an irreducible factor of $\varphi$, and let $\Phi_{0}$ denote the intersection of $\Phi$ with the linear subspace of $i \underline{b}^{*}$ spanned by $\varphi_{0}$. Then $\Phi_{0}$ is a subroot system of $\Phi$ with the same rank as $\varphi_{0}$. Since there are no root sytems of the same rank properly containing a root system of type $A_{1}$ or $B_{2}$, we must have $\varphi_{0}=\Phi_{0}$. Thus $\varphi_{0}$ is a subroot system of $\Phi$.

We want to associate to every $\varphi \in \mathcal{T}(\Phi)$ a group $G_{\varphi}$ which shares certain Cartan subgroups with $G$. Fix $\varphi \in \mathcal{T}(\Phi)$, and write $\varphi=\varphi_{1} \cup \ldots \cup \varphi_{k}$ for its decomposition into irreducible factors. Each $\varphi_{i}, 1 \leq i \leq k$, is a subroot system of $\Phi$ and corresponds to a Lie subalgebra $\underline{g}_{i}$ of $\underline{g}$ as follows.

As in $\S 2$, for each $\alpha \in \Phi$ we have the root space $\left(\underline{g}_{\alpha}\right)_{\mathbf{C}}$ and the normalized root vector $H_{\alpha}^{*} \in\left(\underline{b}_{s}\right)_{\mathbf{C}}$. Now define

$$
\begin{gathered}
\left(\underline{b}_{i}\right)_{\mathbf{C}}=\sum_{\alpha \in \varphi_{i}} \mathbf{C} H_{\alpha}^{*}, \quad \underline{b}_{i}=\underline{b} \cap\left(\underline{b}_{i}\right)_{\mathbf{C}}=\sum_{\alpha \in \varphi_{i}} \mathbf{R} i H_{\alpha}^{*} ; \\
\left(\underline{g}_{i}\right)_{\mathbf{C}}=\left(\underline{b}_{i}\right)_{\mathbf{C}}+\sum_{\alpha \in \varphi_{i}}\left(\underline{g}_{\alpha}\right)_{\mathbf{C}}, \quad \underline{g}_{i}=\underline{g} \cap\left(\underline{g}_{i}\right)_{\mathbf{C}} .
\end{gathered}
$$

Let $\sigma$ be the involution of $\underline{g}_{\mathbf{C}}$ with fixed points $\underline{g}$. For each $\alpha \in \Phi$ we have $\alpha^{\sigma} \in \Phi$ defined by

$$
\alpha^{\sigma}(H)=\overline{\alpha(\sigma H)}, \quad H \in \underline{b}_{\mathbf{C}} .
$$

Since $\alpha$ takes pure imaginary values on $\underline{b}$, we have $\alpha^{\sigma}=-\alpha$ for all $\alpha \in \Phi$. Thus for all $\alpha \in \Phi$,

$$
\left.\sigma\left(H_{\alpha}^{*}\right)=H_{\alpha^{\sigma}}^{*}=H_{-\alpha}^{*}, \quad \sigma\left(\underline{g}_{\alpha}\right)_{\mathbf{C}}\right)=\left(\underline{g}_{\alpha^{\sigma}}\right)_{\mathbf{C}}=\left(\underline{g}_{-\alpha}\right)_{\mathbf{C}} .
$$

Thus $\left(\underline{g}_{i}\right)_{\mathbf{C}}$ is stable under $\sigma$. Now the restriction of $\sigma$ to $\left(\underline{g}_{i}\right)_{\mathbf{C}}$ is an involution with fixed points $\underline{g}_{i}$. Thus $\underline{g}_{i}$ is a real form of $\left(\underline{g}_{i}\right)_{\mathbf{C}}$.

Since $\varphi_{i}$ is of type $A_{1}$ or $B_{2} \simeq C_{2},\left(\underline{g}_{i}\right)_{\mathbf{C}}$ is isomorphic to either $\underline{s l}(2, \mathbf{C})$ or $\underline{s o}(5, \mathbf{C}) \simeq \underline{s p}(4, \mathbf{C})$. Thus $\underline{g}_{i}$ is isomorphic to one of

$$
\underline{s l}(2, \mathbf{R}), \underline{s u}(2), \underline{s o}(3,2), \underline{s o}(4,1), \text { or } \underline{s o}(5) .
$$

We also define

$$
\underline{g}_{0}=\underline{b}_{0}=\{H \in \underline{b}: \alpha(H)=0 \forall \alpha \in \varphi\} \text { and } \varphi_{0}=\emptyset .
$$

Let $G_{i}$ be the connected subgroup of $G$ corresponding to $\underline{g}_{i}, K_{i}=G_{i} \cap K$, $B_{i}=\exp \left(\underline{b}_{i}\right)=G_{i} \cap B$. Let $G_{0} \times G_{1} \times \ldots \times G_{k}$ denote the abstract direct product of the groups $G_{i}, 0 \leq i \leq k$, and define $f: G_{0} \times \ldots \times G_{k} \rightarrow G$ by

$$
f\left(g_{0}, \ldots, g_{k}\right)=g_{0} \ldots g_{k}, \quad g_{i} \in G_{i}, 0 \leq i \leq k .
$$


Here $g_{0} \ldots g_{k}$ denotes the product in $G$ of the elements $g_{i} \in G_{i} \subset G$. Note that $f$ depends on the ordering of the irreducible factors $\varphi_{i}, 1 \leq i \leq k$, and is not necessarily a group homomorphism, since elements from the different subgroups $G_{i}$ do not necessarily commute with each other as elements of $G$. This is because, although roots in different irreducible factors of $\varphi$ are orthogonal to each other, they need not be strongly orthogonal as elements of $\Phi$.

However, since $B_{i} \subset B, 0 \leq i \leq k$, and $B$ is abelian, $f: B_{0} \times \ldots \times B_{k} \rightarrow B$ is a group homomorphism. Let $Z_{B}$ denote the kernel of this homomorphism, and let $Z_{i}$ denote the center of $G_{i}, 0 \leq i \leq k$. The following lemma is easy to check

Lemma 4.1. $f: B_{0} \times \ldots \times B_{k} \rightarrow B$ is surjective and $Z_{B} \subset Z_{0} \times \ldots \times Z_{k}$ is a central subgroup of $G_{0} \times \ldots \times G_{k}$.

Define

$$
\begin{gathered}
G_{\varphi}=\left(G_{0} \times \ldots \times G_{k}\right) / Z_{B}, \quad K_{\varphi}=\left(K_{0} \times \ldots \times K_{k}\right) / Z_{B}, \\
B_{\varphi}=\left(B_{0} \times \ldots \times B_{k}\right) / Z_{B}, \quad Z_{\varphi}=\left(Z_{0} \times \ldots \times Z_{k}\right) / Z_{B} .
\end{gathered}
$$

Then $G_{\varphi}$ and $K_{\varphi}$ are connected reductive Lie groups, $B_{\varphi}$ is a Cartan subgroup of both $G_{\varphi}$ and $K_{\varphi}$, and $Z_{\varphi}$ is the center of $G_{\varphi}$. The Lie algebra $\underline{g}_{\varphi}=\sum_{i=0}^{k} \underline{g}_{i}$ of $G_{\varphi}$ can be identified with a subset, but not necessarily a subalgebra, of $g$. Note that each $\underline{g}_{i}$ is stable under the Cartan involution $\theta$ of $g$ so that the restriction of $\theta$ to $G_{i}$ is a Cartan involution of $G_{i}$. Further, $K_{i}$ is the set of fixed points of $\theta$ in $G_{i}$. Thus $\theta$ induces a Cartan involution of $G_{\varphi}$ with fixed points $K_{\varphi}$.

Let exp denote the exponential mapping from $g$ into $G$, and $\exp _{\varphi}$ denote the exponential mapping of $\underline{g}$ into $G_{\varphi}$. The Cartan subalgebra $\underline{b}=\sum_{i=0}^{k} \underline{b}_{i}$ can be identified with the Lie algebra of $B_{\varphi}$ and is a Cartan subalgebra of $\underline{g}_{\varphi}$ and $\underline{k}_{\varphi}$. Further,

$$
\Phi\left(\left(\underline{g}_{\varphi}\right)_{\mathbf{C}}, \underline{b}_{\mathbf{C}}\right)=\varphi, \quad \Phi\left(\left(\underline{k}_{\varphi}\right)_{\mathbf{C}}, \underline{b}_{\mathbf{C}}\right)=\varphi_{K}=\varphi \cap \Phi_{K} .
$$

The Weyl group $W\left(\varphi_{K}\right)$ generated by reflections in the compact roots $\varphi_{K}=\varphi \cap \Phi_{K}$ of $\varphi$ is naturally a subgroup of $W\left(\Phi_{K}\right)$. For $H \in \underline{b}, b=\exp _{\varphi}(H), v \in W\left(\varphi_{K}\right)$, define $v b=\exp _{\varphi}(v H)$. The following lemma is also routine.

Lemma 4.2. (i) The mapping $f_{B}: B_{\varphi} \rightarrow B$ induced by $f$ is an isomorphism.

(ii) $f_{B}\left(\exp _{\varphi}(H)\right)=\exp (H)$ for all $H \in \underline{b}$.

(iii) $f_{B}(v b)=v f_{B}(b)$ for all $b \in B_{\varphi}, v \in W\left(\varphi_{K}\right)$.

(iv) $Z_{G} \subset f_{B}\left(Z_{\varphi}\right)$.

(v) $K_{\varphi} / Z_{\varphi}$ and $B_{\varphi} / Z_{\varphi}$ are compact.

We have seen that $G_{\varphi}$ together with the subgroups $K_{\varphi}$ and $B_{\varphi}$ satisfies the same hypotheses as our original group $G$ with its subgroups $K$ and $B$. Thus the results of $\S 2$ and $\S 3$ can be applied to the group $G_{\varphi}$.

Remark 4.1. Suppose that $\Phi$ contains no irreducible factors of type $B_{n}, n \geq 3$, or $F_{4}$. Then for any $\varphi \in \mathcal{T}(\Phi)$, roots from different irreducible factors of $\varphi$ are strongly orthogonal. Thus for $0 \leq i \neq j \leq k, G_{i}$ and $G_{j}$ commute inside $G$. Hence $f: G_{0} \times \ldots \times G_{k} \rightarrow G$ is a group homomorphism, and it is easy to see that the kernel of $f$ is $Z_{B}$. Thus $G_{\varphi}$ is embedded as a subgroup of $G$ in this case. 
Now for $\varphi \in \mathcal{T}(\Phi)$ as above, we let $\mathcal{S O}(\varphi)$ denote the set of all subsets of $\varphi$ consisting of noncompact roots which are strongly orthogonal in $\varphi$. Since $\varphi_{K}=$ $\varphi \cap \Phi_{K}$, noncompact roots in $\varphi$ are also noncompact in $\Phi$. Further, the inner product on $\varphi$ is the restriction of the inner product in $\Phi$. However, for $\alpha, \beta \in \varphi$ with $\langle\alpha, \beta\rangle=0, \alpha$ strongly orthogonal to $\beta$ in $\varphi$ means that $\alpha \pm \beta \notin \varphi$, while $\alpha, \beta$ strongly orthogonal in $\Phi$ means $\alpha \pm \beta \notin \Phi$. Thus $\mathcal{S} O(\varphi)$ need not be a subset of $\mathcal{S} O(\Phi)$.

For each noncompact root $\alpha \in \varphi \subset \Phi$ we have the fixed root vectors $X_{\alpha}, Y_{\alpha}$ used to define the Cayley transform $c_{\alpha}$. Then since $X_{\alpha}, Y_{\alpha} \in\left(\underline{g}_{\varphi}\right)_{\mathbf{C}}$,

$$
c_{\varphi, \alpha}=\operatorname{Ad} \exp _{\varphi}(\pi / 4)\left(Y_{\alpha}-X_{\alpha}\right)
$$

is a Cayley transform defined on $\left(\underline{g}_{\varphi}\right)_{\mathbf{C}}$. Now each $S \in \mathcal{S O}(\varphi)$ corresponds as in $\S 2$ to a Cartan subgroup $H_{\varphi, S}=T_{\varphi, S} A_{\varphi, S}$ of $G_{\varphi}$ with Cartan subalgebra $\underline{h}_{\varphi, S}$. Let $S_{i}=S \cap \varphi_{i}, 0 \leq i \leq k$. Then $S=\bigcup_{i} S_{i}$, and each $S_{i}$ is a set of strongly orthogonal noncompact roots in $\varphi_{i}$. Thus $S_{i}$ corresponds to a Cartan subgroup $H_{i, S_{i}}=T_{i, S_{i}} A_{S_{i}}$ of $G_{i}$. Since $Z_{B} \subset Z_{0} \times \ldots \times Z_{k}$, it is contained in every Cartan subgroup of $G_{0} \times \ldots \times G_{k}$, and

$$
H_{\varphi, S}=\left(H_{0, S_{0}} \times \ldots \times H_{k, S_{k}}\right) / Z_{B}
$$

Lemma 4.3. (i) For any $S \in \mathcal{S} O(\varphi)$ and $0 \leq i \leq k, T_{i, S_{i}} \subset B_{i}$. Thus $T_{\varphi, S} \subset B_{\varphi}$.

(ii) Let $S \in \mathcal{S} O(\varphi)$ and $h=t a \in H_{\varphi, S}$. Then

$$
W\left(\varphi_{K}\right) \cap W\left(\varphi_{t}\right)=W\left(\varphi_{K, t}\right) .
$$

Proof. For $i=0, G_{0}=B_{0}$ is abelian, and $S_{0}=\emptyset, T_{0, S_{0}}=B_{0}$. For $1 \leq i \leq k, G_{i}$ is locally isomorphic to one of $S L(2, \mathbf{R}), S U(2), S O(3,2), S O(4,1)$, or $S O(5)$. It is trivial that these properties holds for the compact groups and easy to check that they hold in the other three cases. They are also independent of local isomorphism class.

Every Cartan subgroup $H_{\varphi}$ of $G_{\varphi}$ is conjugate to one of the form $H_{\varphi, S}, S \in$ $\mathcal{S} O(\varphi)$. However if the roots in $S$ are not strongly orthogonal as elements of $\Phi$, $H_{\varphi, S}$ will not correspond to a Cartan subgroup $H_{S}$ of $G$. This is because the groups $A_{i, S_{i}}$ will not commute with each other, and so the restriction of $f$ to $\left(H_{1, S_{1}} \times \ldots \times H_{k, S_{k}}\right)$ is not a group homomorphism. Thus we restrict our attention to strongly orthogonal sets in

$$
\mathcal{S} O(\Phi, \varphi)=\{S \in \mathcal{S} O(\Phi): S \subset \varphi\}=\mathcal{S O}(\Phi) \cap \mathcal{S O}(\varphi) .
$$

Lemma 4.4. Let $S, S^{\prime} \in \mathcal{S} O(\varphi)$ such that $H_{\varphi, S}$ and $H_{\varphi, S^{\prime}}$ are conjugate in $G_{\varphi}$. Then $S \in \mathcal{S} O(\Phi, \varphi)$ if and only if $S^{\prime} \in \mathcal{S} O(\Phi, \varphi)$.

Proof. Since $H_{\varphi, S}$ and $H_{\varphi, S^{\prime}}$ are conjugate in $G_{\varphi}$, by Lemma 2.2 there is $v \in$ $W\left(\varphi_{K}\right)$ such that $v S \equiv S^{\prime}$. Since $W\left(\varphi_{K}\right) \subset W\left(\Phi_{K}\right)$, we know that $S \in \mathcal{S} O(\Phi)$ if and only if $v S \in \mathcal{S} O(\Phi)$. Thus it suffices to show that for $S, S^{\prime} \in \mathcal{S} O(\varphi)$ with $S^{\prime} \equiv S$, we have $S \in \mathcal{S} O(\Phi)$ if and only if $S^{\prime} \in \mathcal{S} O(\Phi)$. But $\varphi$ is the union of irreducible factors $\varphi_{i}$ of type $A_{1}$ or $B_{2}$, and it is easy to see for these two cases that if $S_{i}, S_{i}^{\prime} \in \mathcal{S} O\left(\varphi_{i}\right)$ with $S_{i} \equiv S_{i}^{\prime}$, then $S_{i} \cup-S_{i}=S_{i}^{\prime} \cup-S_{i}^{\prime}$. Thus if $S \equiv S^{\prime}$, we have $S \cup-S=S^{\prime} \cup-S^{\prime}$. Thus $S \in \mathcal{S} O(\Phi)$ if and only if $S^{\prime} \in \mathcal{S} O(\Phi)$.

Recall that $\underline{g} \varphi$ is a subset, although not necessarily a subalgebra, of $\underline{g}$. However, we have the following easy lemma. 
Lemma 4.5. For $S \in \mathcal{S} O(\Phi, \varphi), \underline{h}_{\varphi, S}=\underline{h}_{S}$, and $c_{\varphi, S} H=c_{S} H$ for all $H \in \underline{b}_{\mathbf{C}}$.

Because of Lemma 4.5 we will drop the subscripts $\varphi$ and write $\underline{h}_{S}$ and $c_{S}$ for the Cartan subalgebra and Cayley transform corresponding to $S \in \mathcal{S O} O(\Phi, \varphi)$ in either $\underline{g}$ or $\underline{g}_{\varphi}$.

Lemma 4.6. Let $S \in \mathcal{S} O(\Phi, \varphi)$. For each $0 \leq i \leq k, H_{i, S_{i}}$ is a subgroup of $H_{S}^{1}$. Further, the homomorphism $f_{S}: H_{\varphi, S} \rightarrow H_{S}^{1}$ induced by $f$ is an isomorphism. It satisfies $f_{S}\left(\exp _{\varphi}(H)\right)=\exp H$ for all $H \in \underline{h}_{S}$.

Proof. By definition, $H_{S}=C_{G}\left(\underline{h}_{S}\right)$. Fix $0 \leq i \leq k$. Then $H_{i, S_{i}}=T_{i, S_{i}} A_{i, S_{i}}=$ $C_{G_{i}}\left(\underline{h}_{i, S_{i}}\right)$. Clearly $A_{i, S_{i}}=\exp \underline{a}_{i, S_{i}} \subset \exp \underline{a}_{S}=A_{S}$. Let $t_{i} \in T_{i, S_{i}}$. We know $t_{i}$ centralizes $\underline{h}_{i, S_{i}}$. We must show that it centralizes $\underline{h}_{j, S_{j}}=$ for all $j \neq i$. But by Lemma $4.3, t_{i} \in B_{i}$. Thus it centralizes $\underline{t}_{j, S_{j}} \subset \underline{b}$. Now $\underline{a}_{j, S_{j}}$ is generated by elements of the form $X_{\beta}+Y_{\beta}, \beta \in S_{j}$. Now $t_{i} \in B_{i}=\exp \left(\sum_{\alpha \in \varphi_{i}} i \mathbf{R} H_{\alpha}^{*}\right)$, and $\beta\left(H_{\alpha}^{*}\right)=0$ for all $\beta \in S_{j}, \alpha \in \varphi_{i}$. Thus $t_{i}$ centralizes $\underline{a}_{j, S_{j}}$. We have seen that $H_{i, S_{i}}$ centralizes all of $\underline{h}_{S}$ so that $H_{i, S_{i}}=C_{G_{i}}\left(\underline{h}_{S}\right)=G_{i} \cap H_{S} \subset H_{S}$. By Lemma 4.3, we in fact have $H_{i, S_{i}}=T_{i, S_{i}} A_{i, S_{i}} \subset\left(T_{S} \cap B\right) A_{S}=H_{S}^{1}$.

Since $H_{S}^{1}$ is abelian we know that

$$
f: H_{0, S_{0}} \times \ldots \times H_{k, S_{k}} \rightarrow H_{S}^{1}
$$

is a group homomorphism. Suppose that $\left(h_{0}, \ldots, h_{k}\right)$ is in the kernel of $f$. Write $h_{i}=t_{i} a_{i}$ where $t_{i} \in T_{i, S_{i}}, a_{i} \in A_{i, S_{i}}$. Then $f\left(h_{0}, \ldots, h_{k}\right)=t_{0} \ldots t_{k} a_{0} \ldots a_{k}=1$ implies that $t_{0} \ldots t_{k}=1$ and $a_{0} \ldots a_{k}=1$. Since $t_{i} \in B_{i}$, this implies that $\left(t_{0}, \ldots, t_{k}\right) \in Z_{B}$. But since $A_{S}=A_{0, S_{0}} \ldots A_{k, S_{k}}$ is a direct product, $a_{0} \ldots a_{k}=1$ implies that $a_{i}=$ $1,0 \leq i \leq k$. Thus $\left(h_{0}, \ldots, h_{k}\right)=\left(t_{0}, \ldots, t_{k}\right) \in Z_{B}$.

We have shown that $f_{S}: H_{\varphi, S} \rightarrow H_{S}^{1}$ is injective. Now suppose that $h=t a \in H_{S}^{1}$ where $t \in T_{S} \cap B$ and $a \in A_{S}$. Since $A_{S}=A_{0, S_{0}} \ldots A_{k, S_{k}}$ is a direct product, there are unique $a_{i} \in A_{i, S_{i}}, 0 \leq i \leq k$, such that $a=a_{0} \ldots a_{k}$. Since $t \in B=B_{0} \ldots B_{k}$ there are (not necessarily unique) $b_{i} \in B_{i}, 0 \leq i \leq k$, such that $t=b_{0} \ldots b_{k}$. Let $0 \leq i \leq k$. Then $t=b_{0} \ldots b_{k} \in T_{S}$ centralizes $\underline{h}_{S}$ and hence $\underline{h}_{i, S_{i}}$. But as above, for each $0 \leq j \neq i \leq k$, every $b_{j} \in B_{j}$ centralizes $\underline{h}_{i, S_{i}}$. Thus $b_{i}$ centralizes $\underline{h}_{i, S_{i}}$ and hence is an element of $H_{i, S_{i}} \cap B_{i}=T_{i, S_{i}}$. Thus $h_{i}=b_{i} a_{i} \in H_{i, S_{i}}, 0 \leq i \leq k$, and $f\left(h_{0}, \ldots, h_{k}\right)=t a=h$. Thus $f_{S}$ is surjective.

Finally, for any $H=\sum_{0 \leq i \leq k} H_{i} \in \underline{h}_{S}$,

$$
f_{S}\left(\exp _{\varphi}(H)\right)=f\left(\exp \left(H_{0}\right), \ldots, \exp \left(H_{k}\right)\right)=\exp \left(H_{0}\right) \ldots \exp \left(H_{k}\right)=\exp (H) .
$$

Let $S \in \mathcal{S O}(\varphi), h=t a \in H_{\varphi, S}$. By Lemma 4.3 (ii) we have $W\left(\varphi_{K}\right) \cap W\left(\varphi_{t}\right)=$ $W\left(\varphi_{K, t}\right)$. Thus $u t=t$ for all $u \in W\left(\varphi_{K}\right) \cap W\left(\varphi_{t}\right)$ so that $W_{0}(h)=W\left(\varphi_{K}\right) \cap$ $W\left(\varphi_{t}\right)=W\left(\varphi_{K, t}\right)$. Thus if we define

$$
W\left(\varphi_{K}, h\right)=\left\{[v, s]:(v, s) \in W\left(\varphi_{K}\right) \times W\left(\varphi_{t}\right)\right\}
$$

as in $\S 2$, we have $[v, s]=\left[v^{\prime}, s^{\prime}\right]$ if and only if $v s=v^{\prime} s^{\prime}$. Thus we can identify $W\left(\varphi_{K}, h\right)$ with the set of all elements $w \in W(\varphi)$ such that $w=v s$ for some $v \in W\left(\varphi_{K}\right), s \in W\left(\varphi_{t}\right)$. As in $\S 2$, for $S, S^{\prime} \in \mathcal{S} O(\varphi), h=t a \in H_{\varphi, S}$, we will write $S^{\prime} \equiv_{t} S$ if $S^{\prime} \equiv S$ and $t \in T_{\varphi, S^{\prime}}$. We let $\Lambda(\varphi, h)$ denote the set of all pairs $\left(w, S^{\prime}\right)$ such that $w=v s \in W\left(\varphi_{K}, h\right)$ and $v^{-1} S^{\prime} \equiv_{t} S$. For $h=t a \in$ $H_{\varphi, S},\left(w, S^{\prime}\right) \in \Lambda(\varphi, h), w=v s$, we define $\left(w, S^{\prime}\right) \cdot h=h^{\prime}=t^{\prime} a^{\prime} \in H_{\varphi, S^{\prime}}$ where $t^{\prime}=v t, \log _{\varphi} a^{\prime}=c_{S^{\prime}} w c_{S}^{-1} \log _{\varphi}(a)$. It is easy to check the following. 
Lemma 4.7. Let $S \in \mathcal{S} O(\Phi, \varphi), h^{\prime}=t^{\prime} a^{\prime} \in H_{\varphi, S}, h=t a=f_{S}\left(h^{\prime}\right)$.

(i) $\varphi_{t^{\prime}}=\varphi \cap \Phi_{t}$.

(ii) Let $\left(w, S^{\prime}\right) \in \Lambda\left(\varphi, h^{\prime}\right)$ and write $w=v s, v \in W\left(\varphi_{K}\right), s \in W\left(\varphi_{t^{\prime}}\right)$. Then $S^{\prime} \in \mathcal{S} O(\Phi, \varphi)$ and $v^{-1} S^{\prime} \equiv_{t} S$.

Let $S \in \mathcal{S} O(\Phi, \varphi), h^{\prime}=t^{\prime} a^{\prime} \in H_{\varphi, S}, h=t a=f_{S}\left(h^{\prime}\right)$. Then by Lemma 4.7 (i), $W\left(\varphi_{t^{\prime}}\right) \subset W\left(\Phi_{t}\right)$. Thus for every $v \in W\left(\varphi_{K}\right), s \in W\left(\varphi_{t^{\prime}}\right)$, we have $(v, s) \in$ $W\left(\Phi_{K}\right) \times W\left(\Phi_{t}\right)$. Suppose that $v, v^{\prime} \in W\left(\varphi_{K}\right), s, s^{\prime} \in W\left(\varphi_{t^{\prime}}\right)$, such that $v s=v^{\prime} s^{\prime}$. Then there is $u \in W\left(\varphi_{K}\right) \cap W\left(\varphi_{t^{\prime}}\right)=W\left(\varphi_{K, t^{\prime}}\right) \subset W\left(\Phi_{K, t}\right) \subset W_{0}(h)$ such that $v^{\prime}=v u, s^{\prime}=u^{-1} s$, and so $\left[v^{\prime}, s^{\prime}\right]=[v, s]$ as elements of $W\left(\Phi_{K}, h\right)$. Thus $v s \mapsto$ $[v, s]$ gives a well-defined mapping from $W\left(\varphi_{K}, h^{\prime}\right)$ into $W\left(\Phi_{K}, h\right)$. Suppose that $[v, s]=1$. Then $v=s^{-1} \in W_{0}(h)$. In particular, $v s=1$. Thus the mapping from $W\left(\varphi_{K}, h^{\prime}\right)$ into $W\left(\Phi_{K}, h\right)$ defined by $v s \mapsto[v, s]$ is injective. We will use this mapping to identify $W\left(\varphi_{K}, h^{\prime}\right)$ with a subset of $W\left(\Phi_{K}, h\right)$. Now let $\left(w, S^{\prime}\right) \in$ $\Lambda\left(\varphi, h^{\prime}\right), w=v s$. Then by Lemma 4.7 (ii) we have $S^{\prime} \in \mathcal{S} O(\Phi, \varphi)$, and $v^{-1} S^{\prime} \equiv_{t} S$. Thus $\left([v, s], S^{\prime}\right) \in \Lambda(h)$. Thus we can also identify $\Lambda\left(\varphi, h^{\prime}\right)$ with a subset of $\Lambda(h)$.

Let $G_{\varphi}^{\prime}$ denote the set of regular semisimple elements of $G_{\varphi}$. For any $x \in G_{\varphi}$, let $\mathcal{O}_{\varphi}(x)$ denote the orbit of $x$ in $G_{\varphi}$.

Lemma 4.8. Let $S, S^{\prime} \in \mathcal{S} O(\varphi), h \in H_{\varphi, S} \cap G_{\varphi}^{\prime}, h^{\prime} \in H_{\varphi, S^{\prime}} \cap G_{\varphi}^{\prime}$, such that $\mathcal{O}_{\varphi}(h)=\mathcal{O}_{\varphi}\left(h^{\prime}\right)$. Then $S \in \mathcal{S} O(\Phi, \varphi)$ if and only if $S^{\prime} \in \mathcal{S} O(\Phi, \varphi)$. Further, in the case that $S, S^{\prime} \in \mathcal{S} O(\Phi, \varphi)$, then $\mathcal{O}_{G}\left(f_{S}(h)\right)=\mathcal{O}_{G}\left(f_{S^{\prime}}\left(h^{\prime}\right)\right)$. Finally, let $S \in \mathcal{S} O(\Phi, \varphi), h \in H_{\varphi, S},\left(w, S^{\prime}\right) \in \Lambda(\varphi, h)$. Then $f_{S^{\prime}}\left(\left(w, S^{\prime}\right) \cdot h\right)=\left(w, S^{\prime}\right) \cdot f_{S}(h)$.

Proof. Since $\mathcal{O}_{\varphi}(h)=\mathcal{O}_{\varphi}\left(h^{\prime}\right), H_{\varphi, S}$ and $H_{\varphi, S^{\prime}}$ are conjugate in $G_{\varphi}$. Thus by Lemma 4.4, $S \in \mathcal{S} O(\Phi)$ if and only if $S^{\prime} \in \mathcal{S O}(\Phi)$.

Assume that $S, S^{\prime} \in \mathcal{S} O(\Phi, \varphi)$. Write $h=t a \in H_{\varphi, S}, h^{\prime}=t^{\prime} a^{\prime} \in H_{\varphi, S^{\prime}}$. Since $\mathcal{O}_{\varphi}(h)=\mathcal{O}_{\varphi}\left(h^{\prime}\right)$ we can apply Theorem 2.5 to the group $G_{\varphi}$ to conclude that there are $v \in W\left(\varphi_{K}\right), s \in W\left(\varphi_{t}\right)$ such that $\left(v s, S^{\prime}\right) \in \Lambda(\varphi, h)$ and $h^{\prime}=\left(v s, S^{\prime}\right) \cdot h$. Then

$$
t^{\prime}=v t, \quad S^{\prime} \equiv v S, \quad \log _{\varphi} a^{\prime}=c_{S^{\prime}} v s c_{S}^{-1} \log _{\varphi} a .
$$

Now we can write $f_{S}(h)=f_{B}(t) f_{S}(a)$ where $f_{B}(t) \in T_{S}^{1}$ is the image of $t \in$ $T_{\varphi, S} \subset B_{\varphi}$ under the isomorphism $f_{B}, f_{S}(a)=\exp \left(\log _{\varphi} a\right) \in A_{S}$ and $f_{S^{\prime}}\left(h^{\prime}\right)=$ $f_{B}\left(t^{\prime}\right) f_{S^{\prime}}\left(a^{\prime}\right)$ where $f_{B}\left(t^{\prime}\right) \in T_{S^{\prime}}^{1}, f_{S^{\prime}}\left(a^{\prime}\right)=\exp \left(\log _{\varphi} a^{\prime}\right) \in A_{S^{\prime}}$. Now by Lemma 4.2 , when we consider $W\left(\varphi_{K}\right)$ as a subgroup of $W\left(\Phi_{K}\right), f_{B}\left(t^{\prime}\right)=f_{B}(v t)=v f_{B}(t)$. Further

$$
\log \left(f_{S^{\prime}}\left(a^{\prime}\right)\right)=\log _{\varphi} a^{\prime}=c_{S^{\prime}} v s c_{S}^{-1} \log _{\varphi} a=c_{S^{\prime}} v s c_{S}^{-1} \log \left(f_{S}(a)\right) .
$$

Thus $f_{S^{\prime}}\left(h^{\prime}\right)=\left([v, s], S^{\prime}\right) \cdot f_{S}(h)$, and by Theorem $2.5, f_{S}(h)$ and $f_{S^{\prime}}\left(h^{\prime}\right)$ are conjugate in $G$.

Finally, let $S \in \mathcal{S} O(\Phi, \varphi), h \in H_{\varphi, S},\left(w, S^{\prime}\right) \in \Lambda(\varphi, h)$. Write $w=v s$ and $\left(v s, S^{\prime}\right) \cdot h=t^{\prime} a^{\prime}$. Then by the above argument, $f_{S^{\prime}}\left(h^{\prime}\right)=\left([v, s], S^{\prime}\right) \cdot f_{S}(h)$.

We will use the isomorphisms $f_{S}: H_{\varphi, S} \rightarrow H_{S}^{1}, S \in \mathcal{S} O(\Phi, \varphi)$, to define a correspondence of orbits between $G_{\varphi}$ and $G$. Let $\gamma \in G_{\varphi}^{\prime}$. Then there exist $S \in$ $\mathcal{S} O(\varphi)$ and $h \in H_{\varphi, S}$ (not unique) such that $\mathcal{O}_{\varphi}(\gamma)=\mathcal{O}_{\varphi}(h)$. We will say that $\mathcal{O}_{\varphi}(\gamma)$ is a good orbit in $G_{\varphi}^{\prime}$ if $S \in \mathcal{S} O(\Phi, \varphi)$. In the case that $\mathcal{O}_{\varphi}(\gamma)=\mathcal{O}_{\varphi}(h), h \in$ $H_{\varphi, S}$, is a good orbit, we have the Cartan subgroup $H_{S}$ of $G$ and the isomorphism $f_{S}: H_{\varphi, S} \rightarrow H_{S}$ given by Lemma 4.6. We define

$$
F_{\varphi}\left(\mathcal{O}_{\varphi}(\gamma)\right)=\mathcal{O}_{G}\left(f_{S}(h)\right) .
$$


By Lemma 4.8, the definitions of good orbit and the mapping $F_{\varphi}$ are independent of the choice of $S \in \mathcal{S} O(\varphi)$ and $h \in H_{\varphi, S}$ with $\mathcal{O}_{\varphi}(\gamma)=\mathcal{O}_{\varphi}(h)$.

Fix $S \in \mathcal{S} O(\Phi)$ and $h \in H_{S}^{1} \cap G^{\prime}$. Define

$W\left(\Phi_{K}, \varphi, h\right)=\left\{w \in W\left(\Phi_{K}, h\right): \exists S^{\prime} \in \mathcal{S} O(\Phi, \varphi)\right.$ such that $\left.\left(w, S^{\prime}\right) \in \Lambda(h)\right\}$.

The following two lemmas are an easy consequence of Theorem 2.5 applied to $G$ and $G_{\varphi}$.

Lemma 4.9. Let $w \in W\left(\Phi_{K}, \varphi, h\right)$ and suppose that $S^{\prime}, S^{\prime \prime} \in \mathcal{S} O(\Phi, \varphi)$ such that $\left(w, S^{\prime}\right),\left(w, S^{\prime \prime}\right) \in \Lambda(h)$. Then

$$
\mathcal{O}_{\varphi}\left(f_{S^{\prime}}^{-1}\left(\left(w, S^{\prime}\right) \cdot h\right)\right)=\mathcal{O}_{\varphi}\left(f_{S^{\prime \prime}}^{-1}\left(\left(w, S^{\prime \prime}\right) \cdot h\right)\right)
$$

For $w \in W\left(\Phi_{K}, \varphi, h\right)$, we will write

$$
\mathcal{O}(\varphi, w \cdot h)=\mathcal{O}_{\varphi}\left(f_{S^{\prime}}^{-1}\left(\left(w, S^{\prime}\right) \cdot h\right)\right)
$$

where $S^{\prime} \in \mathcal{S} O(\Phi, \varphi)$ such that $\left(w, S^{\prime}\right) \in \Lambda(h)$. By Lemma 4.9 , it is independent of the choice of $S^{\prime}$.

Lemma 4.10. Let $S \in \mathcal{S} O(\Phi)$ and $h \in H_{S}^{1} \cap G^{\prime}$. Then

$$
F_{\varphi}^{-1}\left(\mathcal{O}_{G}(h)\right)=\left\{\mathcal{O}(\varphi, w \cdot h): w \in W\left(\Phi_{K}, \varphi, h\right)\right\} .
$$

For $w \in W\left(\Phi_{K}, \varphi, h\right)$, let $S^{\prime} \in \mathcal{S} O(\Phi, \varphi)$ such that $\left(w, S^{\prime}\right) \in \Lambda(h)$. We will write $W\left(\varphi_{K}, w \cdot h\right)=W\left(\varphi_{K}, h^{\prime}\right)$ where $h^{\prime}=f_{S^{\prime}}^{-1}\left(\left(w, S^{\prime}\right) \cdot h\right) \in H_{\varphi, S^{\prime}}$. By Lemma 4.7 and the remark preceding Lemma $2.4, \varphi_{S^{\prime}, t^{\prime}}=\varphi \cap \Phi_{S^{\prime}, v t}$ is independent of the choices of $S^{\prime}$, so that $W\left(\varphi_{K}, w \cdot h\right)$ is also independent of this choice.

Let $w_{1} \in W\left(\Phi_{K}, \varphi, h\right), w \in W\left(\varphi_{K}, w_{1} \cdot h\right)$. Then there is an element $w w_{1} \in$ $W\left(\Phi_{K}, h\right)$ defined as in Lemma 2.6.

Lemma 4.11. Let $w_{1} \in W\left(\Phi_{K}, \varphi, h\right)$. Then for any $w \in W\left(\varphi_{K}, w_{1} \cdot h\right), w w_{1} \in$ $W\left(\Phi_{K}, \varphi, h\right)$.

Proof. Write $w_{1}=\left[v_{1}, s_{1}\right]$ where $v_{1} \in W\left(\Phi_{K}\right)$ and $s_{1} \in W\left(\Phi_{t}\right)$ and $w=[v, s]$ where $v \in W\left(\varphi_{K}\right), s \in W\left(\varphi \cap \Phi_{v_{1} t}\right)$. Then as in Lemma 2.6 we have

$$
w w_{1}=\left[v v_{1}, v_{1}^{-1} s v_{1} s_{1}\right]
$$

where $v v_{1} \in W\left(\Phi_{K}\right)$ and $v_{1}^{-1} s v_{1} s_{1} \in W\left(\Phi_{t}\right)$. Let $S_{1} \in \mathcal{S} O(\Phi, \varphi)$ such that $\left(w_{1}, S_{1}\right) \in \Lambda(h)$. Since $S_{1} \in \mathcal{S} O(\Phi, \varphi)$ and $v \in W\left(\varphi_{K}\right), v S_{1} \in \mathcal{S} O(\Phi, \varphi)$. Since $S_{1} \equiv v_{1} S$ and $v_{1} t \in T_{S_{1}}^{1}$, then $v S_{1} \equiv v v_{1} S$ and $v v_{1} t \in T_{v S_{1}}^{1}$. Thus $\left(w w_{1}, v S_{1}\right) \in \Lambda(h)$ and so $w w_{1} \in W\left(\Phi_{K}, \varphi, h\right)$.

We want to characterize exactly which of the orbits $\mathcal{O}(\varphi, w \cdot h), w \in W(\Phi, \varphi, h)$, are equal. For this we need an extra regularity condition. Recall that in (2.1) we defined

$$
H_{S}^{\prime \prime}=\left\{h \in H_{S}^{1} \cap G^{\prime}: \text { if }(w, S) \in \Lambda(h) \text { and }(w, S) \cdot h=h, \text { then } w=1\right\} .
$$

Let $w_{1}, w_{2} \in W\left(\Phi_{K}, \varphi, h\right)$. Then we write $w_{2} \sim_{\varphi, h} w_{1}$, or more simply $w_{2} \sim w_{1}$, if there is $w \in W\left(\varphi_{K}, w_{1} \cdot h\right)$ such that $w_{2}=w w_{1}$.

Lemma 4.12. Let $h \in H_{S}^{\prime \prime}$.

(i) Let $w_{1}, w_{2} \in W\left(\Phi_{K}, \varphi, h\right)$. Then

$$
\mathcal{O}\left(\varphi, w_{1} \cdot h\right)=\mathcal{O}\left(\varphi, w_{2} \cdot h\right)
$$

if and only if $w_{2} \sim_{\varphi, h} w_{1}$. In particular, $w_{2} \sim_{\varphi, h} w_{1}$ is an equivalence relation on $W(\Phi, \varphi, h)$. 
(ii) Let $w_{i}, 1 \leq i \leq p$, be a complete set of representatives for the equivalence classes in $W\left(\Phi_{K}, \varphi, h\right)$ given by $\sim_{\varphi, h}$. Let $\sigma \in W\left(\Phi_{K}, \varphi, h\right)$. Then there are unique $1 \leq i \leq p$ and $w \in W\left(\varphi_{K}, w_{i} \cdot h\right)$ such that $\sigma=w w_{i}$.

Proof. (i) Let $S_{1}, S_{2} \in \mathcal{S O}(\Phi, \varphi)$ such that $\left(w_{i}, S_{i}\right) \in \Lambda(h), i=1,2$. Write

$$
h_{i}^{\prime}=f_{S_{i}}^{-1}\left(\left(w_{i}, S_{i}\right) \cdot h\right), \quad i=1,2 .
$$

Then by definition

$$
\mathcal{O}\left(\varphi, w_{i} \cdot h\right)=\mathcal{O}_{\varphi}\left(f_{S_{i}}^{-1}\left(\left(w_{i}, S_{i}\right) \cdot h\right)\right)=\mathcal{O}_{\varphi}\left(h_{i}^{\prime}\right), \quad i=1,2 .
$$

By Theorem 2.5, $\mathcal{O}_{\varphi}\left(h_{1}^{\prime}\right)=\mathcal{O}_{\varphi}\left(h_{2}^{\prime}\right)$ if and only if there is $w \in W\left(\varphi_{K}, h_{1}^{\prime}\right)=$ $W\left(\varphi_{K}, w_{1} \cdot h\right)$ such that $\left(w, S_{2}\right) \in \Lambda\left(\varphi, h_{1}^{\prime}\right)$ and $h_{2}^{\prime}=\left(w, S_{2}\right) \cdot h_{1}^{\prime}$. Now by Lemmas 2.7 and $4.8, h_{2}^{\prime}=\left(w, S_{2}\right) \cdot h_{1}^{\prime}$ if and only if

$$
\begin{aligned}
h & =\left(w_{2}^{-1}, S\right) \cdot\left(w_{2}, S_{2}\right) \cdot h=\left(w_{2}^{-1}, S\right) \cdot f_{S_{2}}\left(h_{2}^{\prime}\right) \\
& =\left(w_{2}^{-1}, S\right) \cdot\left(w, S_{2}\right) \cdot f_{S_{1}}\left(h_{1}^{\prime}\right)=\left(w_{2}^{-1} w, S\right) \cdot\left(w_{1}, S_{1}\right) \cdot h=\left(w_{2}^{-1} w w_{1}, S\right) \cdot h .
\end{aligned}
$$

Thus $\mathcal{O}_{\varphi}\left(h_{1}^{\prime}\right)=\mathcal{O}_{\varphi}\left(h_{2}^{\prime}\right)$ if and only if there is $w \in W\left(\varphi_{K}, w_{1} \cdot h\right)$ such that

$$
h=\left(w_{2}^{-1} w w_{1}, S\right) \cdot h .
$$

Now since $h \in H_{S}^{\prime \prime}$, this is if and only if $w_{2}^{-1} w w_{1}=1$ so that $w_{2}=w w_{1}$.

(ii) Let $\sigma \in W\left(\Phi_{K}, \varphi, h\right)$. Since $\sim$ is an equivalence relation, there is a unique $1 \leq i \leq p$ so that $\sigma \sim w_{i}$. Thus there is $w \in W\left(\varphi_{K}, w_{i} \cdot h\right)$ such that $\sigma=w w_{i}$. Suppose that $w^{\prime} \in W\left(\varphi_{K}, w_{i} \cdot h\right)$ such that $\sigma=w^{\prime} w_{i}$. Write $w_{i}=\left[v_{i}, s_{i}\right], w=$ $[v, s], w^{\prime}=\left[v^{\prime}, s^{\prime}\right], v_{i} \in W\left(\Phi_{K}\right), s_{i} \in W\left(\Phi_{t}\right), v, v^{\prime} \in W\left(\varphi_{K}\right), s, s^{\prime} \in W\left(\varphi_{v_{i}}\right)$. Then if $w w_{i}=w^{\prime} w_{i}$ we must have $v s v_{i} s_{i}=v^{\prime} s^{\prime} v_{i} s_{i}$ as elements of $W(\Phi)$ so that $v s=v^{\prime} s^{\prime}$. But recall that Lemma 4.3 (ii) implies that $w=[v, s]=w^{\prime}=\left[v^{\prime}, s^{\prime}\right]$ as elements of $W\left(\varphi_{K}, w_{i} \cdot h\right)$ if and only if $v s=v^{\prime} s^{\prime}$.

For $\gamma \in G^{\prime}$, let $X_{\varphi}(\gamma)$ denote a complete set of representatives for the good $G_{\varphi}$ orbits which map to $\mathcal{O}_{G}(\gamma)$ under the orbit correspondence $F_{\varphi}$. As an immediate consequence of Lemmas 4.10 and 4.12 we have the following.

Theorem 4.13. Let $h \in H_{S}^{\prime \prime}$, and let $w_{i}, 1 \leq i \leq p$, be a complete set of representatives for the equivalence classes in $W\left(\Phi_{K}, \varphi, h\right)$ given by $\sim_{\varphi, h}$. For each $1 \leq i \leq p$, fix $S_{i} \in \mathcal{S O}(\Phi, \varphi)$ such that $\left(w_{i}, S_{i}\right) \in \Lambda(h)$. Then we can take

$$
X_{\varphi}(h)=\left\{f_{S_{i}}^{-1}\left(\left(w_{i}, S_{i}\right) \cdot h\right): 1 \leq i \leq p\right\} .
$$

\section{Two-Structures}

In this section we review basic facts about two-structures and prove results which will be needed for the lifting of discrete series characters. As in $\S 4$ we let $\mathcal{T}(\Phi)$ denote the set of all two-structures for $\Phi$.

Fix a choice $\Phi^{+}$of positive roots, and let $\varphi \in \mathcal{T}(\Phi)$. Let $\mathcal{B}$ be the set of positive long roots of $\varphi$, where by a long root of $\varphi$ we mean any root in an $A_{1}$ factor or any long root in a $B_{2} \simeq C_{2}$ factor. Then $\mathcal{B}$ is an orthogonal basis for $\varphi$. Suppose that $\beta \in \Phi$ is orthogonal to every $\alpha \in \mathcal{B}$. Then $\beta$ is orthogonal to $\varphi$ and the reflection $s_{\beta}$ in $\beta$ is an element of $W(\Phi)$ with $\operatorname{det} s_{\beta}=-1$ and $s_{\beta} \varphi^{+}=\varphi^{+}$. This contradicts the definition of two-structures. Thus no root of $\Phi$ is orthogonal to every $\alpha \in \mathcal{B}$.

Choose an ordering $\left\{\alpha_{1}, \ldots, \alpha_{n}\right\}$ of $\mathcal{B}$ which satisfies the following condition. Suppose that two roots of $\mathcal{B}$ are in the same irreducible factor of type $B_{2}$ of $\varphi$. Then they should be adjacent in the ordering so that they are $\alpha_{i}, \alpha_{i+1}$ for some $1 \leq i \leq n-1$. 
Now since they are long roots in a factor of type $B_{2}, \frac{1}{2}\left( \pm \alpha_{i} \pm \alpha_{i+1}\right)$ are roots in $\varphi$. We order $\alpha_{i}, \alpha_{i+1}$ so that $\frac{1}{2}\left(\alpha_{i}-\alpha_{i+1}\right) \in \Phi^{+}$. Since no root of $\Phi$ is orthogonal to every $\alpha \in \mathcal{B}$, we can use the lexicographic ordering with respect to $\mathcal{B}$ to define a new choice of positive roots for $\Phi$ which we will denote by $\Phi^{+}(\mathcal{B})$. Now there is a unique $w \in W(\Phi)$ such that $\Phi^{+}(\mathcal{B})=w \Phi^{+}$. As in page 501 of [9] we define

$$
\epsilon\left(\varphi: \Phi^{+}\right)=\operatorname{det} w .
$$

Lemma 5.1. The definition of $\epsilon\left(\varphi: \Phi^{+}\right)$is independent of the choice of ordering of $\mathcal{B}$ used, so long as the ordering satisfies the above condition for each irreducible factor of $\varphi$ of type $B_{2}$.

Proof. In the case that $\Phi$ is spanned by strongly orthogonal roots so that rank $\Phi=$ rank $\varphi$, problems in page 513 of [9] show how to prove that $\epsilon\left(\varphi: \Phi^{+}\right)$is well-defined. We will modify this proof to prove the result in the cases that $\Phi$ is not spanned by strongly orthogonal roots. We may as well assume that $\Phi$ is irreducible. In this case all roots of $\Phi$ have the same length, so there are no factors of type $B_{2}$ in $\varphi$ and hence no restrictions on the ordering of $\mathcal{B}$.

Let $\left\{\alpha_{1}, \ldots, \alpha_{n}\right\}$ be an ordering of $\mathcal{B}$. Let $\Phi_{1}^{+}$be the positive roots corresponding to this ordering, and let $\Phi_{2}^{+}$be the positive roots corresponding to the ordering where $\alpha_{i}$ and $\alpha_{i+1}$ are interchanged for some $1 \leq i \leq n-1$. If $\Phi_{1}^{+}=w_{1} \Phi^{+}$and $\Phi_{2}^{+}=w_{2} \Phi^{+}$, then $\Phi_{2}^{+}=w_{2} w_{1}^{-1} \Phi_{1}^{+}$. Thus to show $\operatorname{det} w_{1}=\operatorname{det} w_{2}$ it suffices to show that $\Phi_{1}^{+} \cap \Phi_{2}^{-}$contains an even number of roots.

For any $\gamma \in \Phi$, define $c_{i}=c_{i}(\gamma)=\left\langle\alpha_{i}, \gamma\right\rangle /\left\langle\alpha_{i}, \alpha_{i}\right\rangle, 1 \leq i \leq n$. Then $\Phi_{1}^{+} \cap \Phi_{2}^{-}$ consists of all roots $\gamma$ such that $c_{1}=\ldots=c_{i-1}=0, c_{i}>0$, and $c_{i+1}<0$. Thus $\gamma$ has the form

$$
\gamma=c_{i} \alpha_{i}+c_{i+1} \alpha_{i+1}+\lambda
$$

where $\lambda$ is in the real vector space spanned by $\Phi$, is orthogonal to $\alpha_{1}, \ldots, \alpha_{i}, \alpha_{i+1}$, but is not necessarily a root. Further, $\lambda \neq 0$ since $\alpha_{i}$ and $\alpha_{i+1}$ are strongly orthogonal, and all roots of $\Phi$ have the same length. Now

$$
\gamma^{\prime}=-s_{\alpha_{i}} s_{\alpha_{i+1}} \gamma=c_{i} \alpha_{i}+c_{i+1} \alpha_{i+1}-\lambda
$$

is also an element of $\Phi_{1}^{+} \cap \Phi_{2}^{-}$. Thus the roots in $\Phi_{1}^{+} \cap \Phi_{2}^{-}$can be grouped in pairs.

Define

$$
W\left(\varphi: \Phi^{+}\right)=\left\{w \in W(\Phi): w\left(\varphi \cap \Phi^{+}\right) \subset \Phi^{+}\right\} .
$$

The following lemma is easy to check from the definitions.

Lemma 5.2. For any $\sigma \in W\left(\varphi: \Phi^{+}\right)$,

$$
\epsilon\left(\sigma \varphi: \Phi^{+}\right)=\operatorname{det} \sigma \epsilon\left(\varphi: \Phi^{+}\right) .
$$

Further, for any $s \in W(\Phi)$,

$$
\epsilon\left(s \varphi: s \Phi^{+}\right)=\epsilon\left(\varphi: \Phi^{+}\right) .
$$

Theorem 5.3. Suppose that $\Phi$ is irreducible and let $\Phi^{+}$be any choice of positive roots for $\Phi$. Then

$$
\sum_{\varphi \in \mathcal{T}(\Phi)} \epsilon\left(\varphi: \Phi^{+}\right)= \begin{cases}(-1)^{n} & \text { if } \Phi \text { is of type } A_{2 n} \\ 1 & \text { otherwise }\end{cases}
$$


Proof. Suppose that $\Phi_{1}^{+}$and $\Phi_{2}^{+}$are two choices of positive roots. Then there is $w \in W(\Phi)$ such that $\Phi_{2}^{+}=w \Phi_{1}^{+}$, and using Lemma 5.2,

$$
\sum_{\varphi \in \mathcal{T}(\Phi)} \epsilon\left(\varphi: \Phi_{2}^{+}\right)=\sum_{\varphi \in \mathcal{T}(\Phi)} \epsilon\left(\varphi: w \Phi_{1}^{+}\right)=\sum_{\varphi \in \mathcal{T}(\Phi)} \epsilon\left(w^{-1} \varphi: \Phi_{1}^{+}\right)=\sum_{\varphi \in \mathcal{T}(\Phi)} \epsilon\left(\varphi: \Phi_{1}^{+}\right)
$$

since $w$ permutes the elements of $\mathcal{T}(\Phi)$. Thus it is enough to prove the result for one choice of positive roots.

We will prove the result by induction on the rank of $\Phi$. It is obvious when $\Phi$ has rank 0 or 1 . Assume that the rank of $\Phi$ is greater than one, and that the theorem is true for root systems of rank less than that of $\Phi$.

Fix $\varphi_{0} \in \mathcal{T}(\Phi)$ and pick a basis $\mathcal{B}$ of long roots of $\varphi_{0}$. Pick an ordering $\mathcal{B}=$ $\left\{\alpha_{1}, \ldots, \alpha_{n}\right\}$ so that if two roots of $\mathcal{B}$ are in the same irreducible factor of type $B_{2}$ of $\varphi_{0}$, then they are adjacent in the ordering. Let $\Phi^{+}=\Phi^{+}(\mathcal{B})$.

Define $\Phi_{1}=\left\{\beta \in \Phi:\left\langle\beta, \alpha_{i}\right\rangle=0,1 \leq i \leq n-1\right\}, \Phi_{1}^{+}=\Phi_{1} \cap \Phi^{+}$. Then $\alpha_{n} \in \Phi_{1}^{+}$so that $\Phi_{1}^{+} \neq \emptyset$. Let $\alpha$ be a simple root for $\Phi_{1}^{+}$. Then because $\Phi^{+}$is the lexicographic ordering with respect to $\alpha_{1}, \ldots, \alpha_{n}$, it is easy to see that $\alpha$ is also a simple root for $\Phi^{+}$.

If $\Phi$ is spanned by strongly orthogonal roots, then $\alpha_{1}, \ldots, \alpha_{n}$ is an orthogonal basis for the vector space spanned by $\Phi$, so we must have $\Phi_{1}^{+}=\left\{\alpha_{n}\right\}$ and $\alpha=\alpha_{n}$. Suppose $\Phi$ is not spanned by strongly orthogonal roots. Then $\Phi$ is of type $A_{2 n}, n \geq$ $1, A_{2 n-1}, n \geq 2, D_{2 n+1}, n \geq 1$, or $E_{6}$, in which case $n=4$. For any $\beta \in \Phi$, let $\Phi_{\beta}$ denote the set of roots in $\Phi$ orthogonal to $\beta$. If $\Phi$ is of type $A_{2 n}, A_{2 n-1}, D_{2 n+1}$, or $E_{6}$, and $\beta \in \Phi$, then $\Phi_{\beta}$ is of type $A_{2 n-2}, A_{2 n-3}, D_{2 n-1} \times A_{1}$, or $A_{5}$ respectively. Using this we can see that $\Phi_{1}$ is of type $A_{1}$ except when $\Phi$ is of type $A_{2 n}$, in which case it is of type $A_{2}$. Thus $\alpha=\alpha_{n}$ except when $\Phi$ is of type $A_{2 n}$. In this case $\alpha_{n}$ is the unique nonsimple root in $\Phi_{1}^{+}$.

Suppose that $\varphi \in \mathcal{T}(\Phi)$ and $\alpha \notin \varphi$. Then since $\alpha$ is simple for $\Phi^{+}, s_{\alpha}\left(\varphi \cap \Phi^{+}\right) \subset$ $\Phi^{+}$so that by Lemma 5.2 ,

$$
\epsilon\left(s_{\alpha} \varphi: \Phi^{+}\right)=-\epsilon\left(\varphi: \Phi^{+}\right) .
$$

Thus such two-structures appear in pairs which cancel in the sum, and so

$$
\sum_{\varphi \in \mathcal{T}(\Phi)} \epsilon\left(\varphi: \Phi^{+}\right)=\sum_{\varphi \in \mathcal{T}(\Phi, \alpha)} \epsilon\left(\varphi: \Phi^{+}\right)
$$

where $\mathcal{T}(\Phi, \alpha)=\{\varphi \in \mathcal{T}(\Phi): \alpha \in \varphi\}$

Let $\varphi \in \mathcal{T}(\Phi, \alpha)$ and suppose that $\varphi_{\alpha}=\varphi \cap \Phi_{\alpha} \notin \mathcal{T}\left(\Phi_{\alpha}\right)$. (Here as above, $\Phi_{\alpha}$ denotes the roots in $\Phi$ orthogonal to $\alpha$.) Using the techniques of Lemmas 4.8 and 4.9 of 5 , we see that such two-structures appear in pairs which cancel in the sum. Thus in any case we have

$$
\sum_{\varphi \in \mathcal{T}(\Phi)} \epsilon\left(\varphi: \Phi^{+}\right)=\sum_{\varphi \in \mathcal{T}(\Phi, \alpha)^{\prime}} \epsilon\left(\varphi: \Phi^{+}\right)
$$

where $\mathcal{T}(\Phi, \alpha)^{\prime}=\left\{\varphi \in \mathcal{T}(\Phi, \alpha): \varphi_{\alpha} \in \mathcal{T}\left(\Phi_{\alpha}\right)\right\}$

Suppose that $\Phi$ is spanned by strongly orthogonal roots. Then it is proven in Lemmas 3.3 and 4.10 of [5] that $\varphi \mapsto \varphi_{\alpha}$ gives a bijection between $\mathcal{T}(\Phi, \alpha)^{\prime}$ and $\mathcal{T}\left(\Phi_{\alpha}\right)$, and that

$$
\epsilon\left(\varphi: \Phi^{+}\right)=\epsilon\left(\varphi_{\alpha}: \Phi_{\alpha}^{+}\right)
$$


for all $\varphi \in \mathcal{T}(\Phi, \alpha)^{\prime}$, where $\Phi_{\alpha}^{+}=\Phi^{+} \cap \Phi_{\alpha}$. Thus the theorem follows from our induction hypothesis applied to the root system $\Phi_{\alpha}$ since $\Phi_{\alpha}$ is spanned by strongly orthogonal roots and hence contains no irreducible factors of type $A_{2 n}$.

Thus we may as well assume that all roots of $\Phi$ are of the same length so that every $\varphi \in \mathcal{T}(\Phi)$ is of type $A_{1}^{n}$. In this case, when $\alpha \in \varphi$ we have $\varphi=\varphi_{\alpha} \cup\{ \pm \alpha\}$. Clearly $\varphi_{\alpha}$ is always in $\mathcal{T}\left(\Phi_{\alpha}\right)$ in this case, and any $\psi_{\alpha} \in \mathcal{T}\left(\Phi_{\alpha}\right)$ can be expanded by adding $\pm \alpha$ to become an element of $\mathcal{T}(\Phi)$. Thus the mapping $\varphi \mapsto \varphi_{\alpha}$ gives a bijection between $\mathcal{T}(\Phi, \alpha)$ and $\mathcal{T}\left(\Phi_{\alpha}\right)$.

Let $\varphi_{0}$ be the two-structure used above to define $\Phi^{+}$. Then by definition $\epsilon\left(\varphi_{0}: \Phi^{+}\right)=1$. If $\Phi$ is not of type $A_{2 n}$, then $\alpha=\alpha_{n}$ so that $\varphi_{0} \in \mathcal{T}(\Phi, \alpha)$. Write $\varphi_{1}=\varphi_{0}$ in this case. Now suppose that $\Phi$ is of type $A_{2 n}$. Recall $\Phi_{1}$ is of type $A_{2}$ and that $\alpha$ is a simple root for $\Phi_{1}^{+}$while $\alpha_{n}$ is the unique nonsimple root in $\Phi_{1}^{+}$. Let $\alpha^{\prime}$ denote the other simple root in $\Phi_{1}^{+}$, and define $\varphi_{1}=s_{\alpha^{\prime}} \varphi_{0}$. Then $s_{\alpha^{\prime}} \alpha_{i}=\alpha_{i}, 1 \leq i \leq n-1$, while $s_{\alpha^{\prime}} \alpha_{n}=\alpha$. Thus $\varphi_{1} \in \mathcal{T}(\Phi, \alpha)$ and $s_{\alpha^{\prime}} \varphi_{0}^{+}=\varphi_{1}^{+}$ so that $\epsilon\left(\varphi_{1}: \Phi^{+}\right)=-1$.

In either case, we let $\psi_{1}=\left(\varphi_{1}\right)_{\alpha}$. Then $\epsilon\left(\psi_{1}: \Phi_{\alpha}^{+}\right)=1$ since $\Phi_{\alpha}^{+}$is the lexicographic ordering with respect to the basis $\left\{\alpha_{1}, \ldots, \alpha_{n-1}\right\}$ of $\psi_{1}$. Now let $\varphi \in \mathcal{T}(\Phi, \alpha)$. Since $\varphi_{\alpha} \in \mathcal{T}\left(\Phi_{\alpha}\right)$, there is $\sigma \in W\left(\Phi_{\alpha}\right)$ such that $\left(\varphi_{\alpha} \cap \Phi^{+}\right)=\sigma\left(\psi_{1} \cap \Phi^{+}\right)$. Now $\left(\varphi_{1} \cap \Phi^{+}\right)=\left(\psi_{1} \cap \Phi^{+}\right) \cup\{\alpha\}$ and $\sigma \alpha=\alpha$, so that $\sigma\left(\varphi_{1} \cap \Phi^{+}\right)=\left(\varphi_{\alpha} \cap \Phi^{+}\right) \cup\{\alpha\}=$ $\varphi \cap \Phi^{+}$. Thus

$$
\epsilon\left(\varphi: \Phi^{+}\right)=\operatorname{det} \sigma \epsilon\left(\varphi_{1}: \Phi^{+}\right) \text {and } \epsilon\left(\varphi_{\alpha}: \Phi_{\alpha}^{+}\right)=\operatorname{det} \sigma \epsilon\left(\psi_{1}: \Phi_{\alpha}^{+}\right) .
$$

Now if $\Phi$ is not of type $A_{2 n}$, then $\epsilon\left(\varphi_{1}: \Phi^{+}\right)=\epsilon\left(\psi_{1}: \Phi_{\alpha}^{+}\right)=1$ so that $\epsilon\left(\varphi: \Phi^{+}\right)=$ $\epsilon\left(\varphi_{\alpha}: \Phi_{\alpha}^{+}\right)$for all $\varphi \in \mathcal{T}(\Phi, \alpha)$. If $\Phi$ is of type $A_{2 n}$, then

$$
\epsilon\left(\varphi_{1}: \Phi^{+}\right)=-1=-\epsilon\left(\psi_{1}: \Phi_{\alpha}^{+}\right)
$$

so that $\epsilon\left(\varphi: \Phi^{+}\right)=-\epsilon\left(\varphi_{\alpha}: \Phi_{\alpha}^{+}\right)$for all $\varphi \in \mathcal{T}(\Phi, \alpha)$. The theorem now follows from our induction hypothesis applied to the root system $\Phi_{\alpha}$ since $\Phi_{\alpha}$ contains no irreducible factors of type $A_{2 n}$ unless $\Phi$ is of type $A_{2 n}$, while in this case $\Phi_{\alpha}$ is of type $A_{2(n-1)}$.

Remark 5.1. A different definition of the signs $\epsilon\left(\varphi: \Phi^{+}\right)$was given in [4]. This definition satisfies Lemma 5.2 , but $\sum_{\varphi \in \mathcal{T}(\Phi)} \epsilon\left(\varphi: \Phi^{+}\right)=1$ for every root system $\Phi$. Thus the two definitions agree unless $\Phi$ contains an irreducible factor of type $A_{2 k}, k \equiv 1(\bmod 2)$, in which case they give opposite signs.

Let $\Phi^{+}$be any choice of positive roots for $\Phi$, and for $\varphi \in \mathcal{T}(\Phi)$, let $\varphi^{+}=\varphi \cap \Phi^{+}$. Then we define

$$
\rho\left(\Phi^{+}\right)=\frac{1}{2} \sum_{\alpha \in \Phi^{+}} \alpha, \quad \rho\left(\varphi^{+}\right)=\frac{1}{2} \sum_{\alpha \in \varphi^{+}} \alpha, \quad \rho\left(\Phi^{+}, \varphi\right)=\rho\left(\Phi^{+}\right)-\rho\left(\varphi^{+}\right) .
$$

Let $\mathcal{B}$ be a basis of long roots for $\varphi$, and let $\mathcal{B}=\left\{\alpha_{1}, \ldots, \alpha_{n}\right\}$ be an ordering so that if two roots of $\mathcal{B}$ are in the same irreducible factor of type $B_{2}$ of $\varphi$, then they are adjacent in the ordering. Let $\Phi^{+}=\Phi^{+}(\mathcal{B}), \varphi^{+}=\varphi \cap \Phi^{+}$.

Lemma 5.4. Assume that $\Phi$ is irreducible and let $\varphi \in \mathcal{T}(\Phi)$. Then

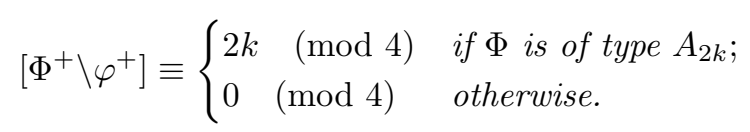


Further, if $\Phi$ is not of type $A_{2 k}$ or $C_{n}, n$ odd, then

$$
\rho\left(\Phi^{+}, \varphi\right)=\sum_{i=1}^{n} n_{i} \alpha_{i}
$$

where $n_{i} \in \mathbf{Z}, 1 \leq i \leq n$. Suppose that $\Phi$ is of type $C_{2 k+1}, k \geq 0$. Then $\varphi$ has a unique irreducible factor of type $A_{1}$. If the root from this irreducible factor is first in the ordering, then again we have

$$
\rho\left(\Phi^{+}, \varphi\right)=\sum_{i=1}^{n} n_{i} \alpha_{i}
$$

where $n_{i} \in \mathbf{Z}, 1 \leq i \leq n=2 k+1$. If this root is last in the ordering, then

$$
\rho\left(\Phi^{+}, \varphi\right)=\frac{1}{2}\left(\sum_{i=1}^{2 k} \alpha_{i}\right)+\sum_{i=1}^{n} n_{i} \alpha_{i}
$$

where $n_{i} \in \mathbf{Z}, 1 \leq i \leq n=2 k+1$.

Proof. Suppose first that $\Phi$ is of type $A_{2 k}, k \geq 1$. Then $\left[\Phi^{+}\right]=k(2 k+1)$ and $\varphi$ is of type $A_{1}^{k}$ so that $\left[\varphi^{+}\right]=k$. Thus $\left[\Phi^{+} \backslash \varphi^{+}\right]=2 k^{2} \equiv 2 k(\bmod 4)$.

Suppose that $\Phi$ is of type $G_{2}$. Then $\varphi=\left\{ \pm \alpha_{1}, \pm \alpha_{2}\right\}$ where $\alpha_{1}$ is long, $\alpha_{2}$ is short, and $\left\langle\alpha_{1}, \alpha_{2}\right\rangle=0$. Now

$$
\Phi \backslash \varphi=\left\{ \pm \frac{1}{2} \alpha_{1} \pm \frac{1}{2} \alpha_{2}, \quad \pm \frac{1}{2} \alpha_{1} \pm \frac{3}{2} \alpha_{2}\right\} .
$$

Thus $\left[\Phi^{+} \backslash \varphi^{+}\right]=4$, and if $\mathcal{B}=\left\{\alpha_{1}, \alpha_{2}\right\}$, then $\rho\left(\Phi^{+}\right)-\rho\left(\varphi^{+}\right)=\alpha_{1}$, while if $\mathcal{B}=\left\{\alpha_{2}, \alpha_{1}\right\}$, then $\rho\left(\Phi^{+}\right)-\rho\left(\varphi^{+}\right)=2 \alpha_{2}$.

Now assume that $\Phi$ is not of type $A_{2 k}$ or $G_{2}$. For any $\gamma \in \Phi$, define

$$
c_{i}(\gamma)=\left\langle\alpha_{i}, \gamma\right\rangle /\left\langle\alpha_{i}, \alpha_{i}\right\rangle, \quad 1 \leq i \leq n .
$$

Since $\Phi$ is not of type $G_{2}$ we have $c_{i} \in\{0, \pm 1 / 2, \pm 1\}, 1 \leq i \leq n$.

Fix $\gamma \in \Phi^{+}$. Since no root of $\Phi$ is orthogonal to every element of $\mathcal{B}$, there is at least one $i, 1 \leq i \leq n$, such that $c_{i}(\gamma) \neq 0$. Suppose that there is only one such index. That is, there is $1 \leq r \leq n$ such that $c_{r}(\gamma) \neq 0, c_{i}(\gamma)=0, i \neq r$. Then as in the proof of Theorem 5.3, since $\Phi$ is not of type $A_{2 n}$, the set of roots in $\Phi$ orthogonal to $(n-1)$ orthogonal roots must be of type $A_{1}$. Thus $\gamma=\alpha_{r} \in \varphi^{+}$.

Fix $\gamma \in \Phi^{+} \backslash \varphi^{+}$. Then by the above, there are at least two indices $i, 1 \leq i \leq n$, such that $c_{i}=c_{i}(\gamma) \neq 0$. Let $r<s$ be the two smallest such indices. Then we can write

$$
\gamma=c_{r} \alpha_{r}+c_{s} \alpha_{s}+\gamma^{\prime}
$$

where $c_{r}>0, c_{s} \neq 0$, and $\gamma^{\prime}$ is in the real vector space spanned by $\Phi$, is orthogonal to $\alpha_{i}, 1 \leq i \leq s$, but is not necessarily a root. Since $c_{r}>0$, we must have $c_{r} \in\{1 / 2,1\}$. Now define

$$
\begin{gathered}
\gamma_{1}=\gamma=c_{r} \alpha_{r}+c_{s} \alpha_{s}+\gamma^{\prime}, \quad \gamma_{2}=-s_{\alpha_{r}} \gamma=c_{r} \alpha_{r}-c_{s} \alpha_{s}-\gamma^{\prime}, \\
\gamma_{3}=s_{\alpha_{s}} \gamma=c_{r} \alpha_{r}-c_{s} \alpha_{s}+\gamma^{\prime}, \quad \gamma_{4}=-s_{\alpha_{s}} s_{\alpha_{r}} \gamma=c_{r} \alpha_{r}+c_{s} \alpha_{s}-\gamma^{\prime} .
\end{gathered}
$$

Since $\alpha_{r}, \alpha_{s} \in \varphi$ and $\gamma \notin \varphi$, we have $\gamma_{i} \notin \varphi, 1 \leq i \leq 4$. Thus $\gamma_{i} \in \Phi^{+} \backslash \varphi^{+}$for all $1 \leq i \leq 4$. 
Suppose that $\gamma^{\prime} \neq 0$. Then the roots $\gamma_{i}, 1 \leq i \leq 4$ are distinct. Further,

$$
\frac{1}{2} \sum_{i=1}^{4} \gamma_{i}=2 c_{r} \alpha_{r}, \quad 2 c_{r} \in\{1,2\}
$$

and so is an integral multiple of $\alpha_{r}$. Thus roots of this type in $\Phi^{+} \backslash \varphi^{+}$can be grouped into subsets of four roots so that the half sum of each group is an integral multiple of $\alpha_{i}$ for some $1 \leq i \leq n$.

Now assume that $\gamma^{\prime}=0$. Then $\gamma=c_{r} \alpha_{r}+c_{s} \alpha_{s}$ is in the linear span of the orthogonal roots $\alpha_{r}, \alpha_{s}$. This cannot happen if all roots of $\Phi$ are of the same length. Thus the lemma is proven in the equal length case.

Now assume that $\Phi$ is of type $B_{n}, C_{n}$, or $F_{4}$, and $\gamma \in \Phi^{+} \backslash \varphi^{+}$such that $\gamma=$ $c_{r} \alpha_{r}+c_{s} \alpha_{s}$. Now since $\alpha_{r}, \alpha_{s}$ are strongly orthogonal, we see that $\gamma$ can only be a root if $\alpha_{r}, \alpha_{s}$ are both long, $\gamma$ is short, and $c_{r}=1 / 2, c_{s}= \pm 1 / 2$. But if $\Phi$ is of type $B_{n}$, all short roots are elements of $\varphi$. Thus we may as well assume that $\Phi$ is of type $C_{n}$ or $F_{4}$.

Assume that $\Phi$ is of type $C_{n}, n \geq 1$, or $F_{n}, n=4$. Then the short roots of $\Phi^{+}$ are all roots of the form $\frac{1}{2}\left(\alpha_{i} \pm \alpha_{j}\right), 1 \leq i<j \leq n$. Let $k$ be the greatest integer in $n / 2$. Then there are $2 k$ short roots in $\varphi^{+}$. Thus if $n$ is even, there are $4 k(k-1)$ short roots in $\Phi^{+} \backslash \varphi^{+}$, while if $n$ is odd, there are $4 k^{2}$ short roots in $\Phi^{+} \backslash \varphi^{+}$.

Suppose that $n$ is even, or $n$ is odd and the root from the irreducible factor of type $A_{1}$ of $\varphi$ is last in the ordering. Then $\gamma \in \varphi^{+}$just in case $\gamma=\frac{1}{2}\left(\alpha_{2 i-1} \pm \alpha_{2 i}\right)$ for some $1 \leq i \leq k$. Thus the half sum of short roots in $\Phi^{+} \backslash \varphi^{+}$is

$$
\frac{1}{2} \sum_{1 \leq j \leq n}(n-j) \alpha_{j}-\frac{1}{2} \sum_{1 \leq i \leq k} \alpha_{2 i-1}=\sum_{1 \leq i \leq k}(n-2 i)\left(\frac{1}{2}\right)\left(\alpha_{2 i-1}+\alpha_{2 i}\right) .
$$

If $n=2 k$ is even, then $\frac{1}{2}(n-2 i)=k-i \in \mathbf{Z}, 1 \leq i \leq k$, while if $n=2 k+1$ is odd, then $\frac{1}{2}(n-2 i)=k-i+1 / 2$.

Now suppose that $n$ is odd and the root from the irreducible factor of type $A_{1}$ of $\varphi_{0}$ is first in the ordering. Then $\gamma \in \varphi^{+}$just in case $\gamma=\frac{1}{2}\left(\alpha_{n-2 i-1} \pm \alpha_{n-2 i}\right)$ for some $0 \leq i \leq k-1$, where $k$ is the greatest integer in $n / 2$. Thus the half sum of short roots in $\Phi^{+} \backslash \varphi^{+}$is

$$
\frac{1}{2} \sum_{1 \leq j \leq n}(n-j) \alpha_{j}-\frac{1}{2} \sum_{0 \leq i \leq k-1} \alpha_{n-2 i-1}=\sum_{0 \leq j \leq k-1} j \alpha_{n-2 j-1}+\sum_{1 \leq j \leq k} j \alpha_{n-2 j} .
$$

Write $r(\Phi)=([\Phi]-[\varphi]) / 4$ where $\varphi \in \mathcal{T}(\Phi)$. Then $r(\Phi)$ is independent of $\varphi$ since all $\varphi \in \mathcal{T}(\Phi)$ are conjugate via $W(\Phi)$, and is an integer by Lemma 5.4.

Theorem 5.5. Let $\Phi^{+}$be any choice of positive roots for $\Phi$. Then

$$
\sum_{\varphi \in \mathcal{T}(\Phi)} \epsilon\left(\varphi: \Phi^{+}\right)=(-1)^{r(\Phi)}
$$

Let $\varphi \in \mathcal{T}(\Phi), \varphi^{+}=\varphi \cap \Phi^{+}$.

(i) Suppose that $\Phi$ is of type $A_{2 n}, n \geq 1$. Then $\rho\left(\Phi^{+}\right)-\rho\left(\varphi^{+}\right)$is not in the weight lattice of $\Phi$.

(ii) Suppose that $\Phi$ contains no irreducible factors of type $A_{2 n}, n \geq 1$. Then $\rho\left(\varphi^{+}\right)$ is in the weight lattice of $\Phi$ and $\rho\left(\Phi^{+}\right)-\rho\left(\varphi^{+}\right)$is in the root lattice of $\Phi$. 
Proof. The first result follows directly from combining Theorem 5.3 and Lemma 5.4 , and (i) is easy to check. It remains to prove (ii). Since $\rho\left(\Phi^{+}\right)$is in the weight lattice of $\Phi$, the first statement will follow from the second. But by Lemma 5.4 there is a choice $\Phi_{1}^{+}$of positive roots for $\Phi$ so that $\rho\left(\Phi_{1}^{+}\right)-\rho\left(\varphi \cap \Phi_{1}^{+}\right)$is in the root lattice of $\varphi$, hence in the root lattice of $\Phi$. But $\rho\left(\Phi^{+}\right)-\rho\left(\varphi^{+}\right)$differs from $\rho\left(\Phi_{1}^{+}\right)-\rho\left(\varphi \cap \Phi_{1}^{+}\right)$by elements of the root lattice of $\Phi$.

Define rank $\mathcal{T}(\Phi)$ to be the common rank of every $\varphi \in \mathcal{T}(\Phi)$. Let $\mathcal{T}_{\text {aug }}(\Phi)$ denote the set of all root subsystems $\varphi \subset \Phi$ such that every irreducible factor of $\varphi$ is of type $A_{1}$ or $B_{2}$ and $\operatorname{rank} \varphi=\operatorname{rank} \mathcal{T}(\Phi)$. Thus $\mathcal{T}(\Phi) \subset \mathcal{T}_{\text {aug }}(\Phi)$.

Lemma 5.6. Let $\varphi \in \mathcal{T}_{\text {aug }}(\Phi)$. Then there is at least one $\varphi_{0} \in \mathcal{T}(\Phi)$ such that $\varphi \subset \varphi_{0}$. Moreover, when $\Phi$ is irreducible, $\varphi_{0}$ is unique unless:

(i) $\Phi$ is of type $B_{n}$ and $\varphi$ contains at least 3 irreducible factors of type $A_{1}$ generated by short roots;

(ii) $\Phi$ is of type $C_{n}$ and $\varphi$ contains at least 3 irreducible factors of type $A_{1}$ generated by long roots;

(iii) $\Phi$ is of type $F_{4}$ and $\varphi$ contains 4 irreducible factors of type $A_{1}$ generated by roots of the same length.

Finally, if $\varphi \notin \mathcal{T}(\Phi)$ then there are two irreducible factors of $\varphi$ of type $A_{1}$ which generate a root system of type $B_{2}$ in $\Phi$.

Proof. We may as well assume that $\Phi$ is irreducible. If $\Phi$ contains no root systems of type $B_{2}$, then augmented two-structures $\varphi$ of $\Phi$ must be of type $A_{1}^{n}$ where $n=$ rank $\mathcal{T}(\Phi)$. But all such $\varphi$ are two-structures. Thus in this case $\mathcal{T}_{\text {aug }}(\Phi)=\mathcal{T}(\Phi)$ and the lemma is trivial. If $\Phi$ contains root systems of type $B_{2}$, then $\Phi$ is of type $B_{n}, C_{n}$, or $F_{4}$. In these cases it is easy to check the lemma using the following facts. If $\Phi$ is of type $B_{n}$, then any long root of $\Phi$ is contained in a unique irreducible factor of $\Phi$ of type $B_{2}$, while any pair of orthogonal short roots span an irreducible factor of type $B_{2}$. If $\Phi$ is of type $C_{n}$, then any short root of $\Phi$ is contained in a unique irreducible factor of $\Phi$ of type $B_{2}$, while any pair of orthogonal long roots span an irreducible factor of type $B_{2}$. Finally, when $\Phi$ is of type $F_{4}$, any two orthogonal roots of the same length determined an irreducible factor of type $B_{2}$.

The following lemma is elementary.

Lemma 5.7. (i) Let $\varphi$ be a root subsystem of $\Phi$ such that every irreducible factor is of type $A_{1}$ or $B_{2}$. Then $\varphi \in \mathcal{T}_{\text {aug }}(\Phi)$ if and only if no root of $\Phi$ is orthogonal to every root in $\varphi$.

(ii) Let $S$ be any set of orthogonal roots in $\Phi$. Then there is $\varphi \in \mathcal{T}(\Phi)$ such that $S \subset \varphi$.

\section{Lifting of Discrete Series}

In this section we will define lifting from the two-structure groups $G_{\varphi}$ to $G$ and restate Theorem 1.2 more precisely as Theorem 6.4. We then prove Theorem 6.4, modulo the proof of Lemma 6.8, which is deferred to the next section.

As in $\S 1$, for $S \in \mathcal{S} O(\Phi, \varphi), h \in H_{\varphi, S}$, such that $f_{S}(h) \in G^{\prime}$, we define

$$
D_{\varphi}^{G}(h)=\left|D_{G}\left(f_{S}(h)\right)\right|^{-\frac{1}{2}}\left|D_{\varphi}(h)\right|^{\frac{1}{2}} .
$$


Let $\mathcal{O}_{\varphi}$ be a good orbit in $G_{\varphi}^{\prime}$ such that $F_{\varphi}\left(\mathcal{O}_{\varphi}\right)$ is a regular semisimple orbit in $G$. Then there are $S \in \mathcal{S} O(\Phi, \varphi), h \in H_{\varphi, S}$, such that $h \in \mathcal{O}_{\varphi}$. Now for any $\gamma \in \mathcal{O}_{\varphi}$ we can define

$$
D_{\varphi}^{G}(\gamma)=D_{\varphi}^{G}(h)
$$

It is easy to see that this is independent of the choice of $S$ and $h$.

As in $\S 4$, for $g \in G^{\prime}$, we let $X_{\varphi}(g)$ denote a complete set of representatives for the good $G_{\varphi}$ orbits which map to $\mathcal{O}_{G}(g)$ under the orbit correspondence $F_{\varphi}$. It is possible that $X_{\varphi}(g)=\emptyset$.

Let $\Theta$ be a class function defined on $G_{\varphi}^{\prime}$. Now for $g \in G^{\prime}$, we define

$$
\left(\operatorname{Lift}_{\varphi}^{G} \Theta\right)(g)=\sum_{h^{\prime} \in X_{\varphi}(g)} D_{\varphi}^{G}\left(h^{\prime}\right) \Theta\left(h^{\prime}\right) .
$$

In the case that $X_{\varphi}(g)=\emptyset$, we have $\left(\operatorname{Lift}_{\varphi}^{G} \Theta\right)(g)=0$. The definition does not depend on the choice of $X_{\varphi}(g)$. If $g, g^{\prime} \in G^{\prime}$ with $\mathcal{O}_{G}(g)=\mathcal{O}_{G}\left(g^{\prime}\right)$ we can take $X_{\varphi}(g)=X_{\varphi}\left(g^{\prime}\right)$. Thus $\operatorname{Lift}_{\varphi}^{G} \Theta$ is a class function on $G^{\prime}$.

Lemma 6.1. (i) Suppose that $\Phi$ contains no irreducible factors of type $A_{2 k}, k \geq$ 1. Let $\lambda \in i \underline{b}^{*}$ be a discrete series parameter for $G$. Then $\lambda$ is also a discrete series parameter for $G_{\varphi}$ for every $\varphi \in \mathcal{T}(\Phi)$. Further, if $\lambda$ is a limit of discrete series parameter for $G$, then it is either a discrete series or limit of discrete series parameter for $G_{\varphi}, \varphi \in \mathcal{T}(\Phi)$.

(ii) Suppose that $\Phi$ is irreducible of type $A_{2 k}, k \geq 1$. Then there is $\varphi \in \mathcal{T}(\Phi)$ such that no discrete series parameter or limit of discrete series parameter for $G$ is a discrete series or limit of discrete series parameter for $G_{\varphi}$.

Proof. (i) follows easily from Theorem 5.5.

(ii) Using the classification in [9] for example, we see that $\Phi_{K}$ is of type $A_{p-1} \times$ $A_{q-1}$ for some $p$ and $q$ with $p+q=2 k+1$. Suppose that $\lambda$ is a discrete series or limit of discrete series parameter for $G$. Then $\lambda-\rho_{G} \in L_{B}$. It is easy to check that when $\Phi$ is of type $A_{2 k}, \rho_{G}$ is in the root lattice of $\Phi$, and hence is an element of $L_{B}$. Thus $\lambda \in L_{B}$. Now $\lambda-\rho_{\varphi} \in L_{B_{\varphi}}=L_{B}$ if and only if $\rho_{\varphi} \in L_{B}$. Thus it suffices to find $\varphi \in \mathcal{T}(\Phi)$ such that $\rho_{\varphi} \notin L_{B}$.

Now $\Phi_{K}=\emptyset$ only if $p \leq 1$ and $q \leq 1$. But $p+q \geq 3$, so this cannot occur. Thus $\Phi_{K} \neq \emptyset$. Fix $\alpha \in \Phi_{K}, \varphi \in \mathcal{T}(\Phi)$. Since by Theorem 5.5(ii) $\rho_{\varphi}$ is not a weight of $\Phi$, there is $\beta \in \Phi$ such that $2\left\langle\rho_{\varphi}, \beta\right\rangle /\langle\beta, \beta\rangle \notin \mathbf{Z}$. Now since $\Phi$ is irreducible, there is $w \in W(\Phi)$ such that $\alpha=w \beta$. Now $w \varphi \in \mathcal{T}(\Phi)$ and $\rho_{w \varphi}$ is not a weight for $\Phi_{K}$, and so $\rho_{w \varphi} \notin L_{B}$.

Fix a discrete series or limit of discrete series parameter $\lambda$ for $G$ and fix $E^{*}=$ $E^{*}(\lambda)$ as in $\S 3$ so that $\lambda$ is in the closure of $E^{*}$. As in $\S 3$ we have the discrete series or limit of discrete series character $\Theta_{\lambda}$ corresponding to $\lambda$ and $E^{*}$. For any $\varphi \in \mathcal{T}(\Phi), E^{*}$ is contained in a unique connected component $E_{\varphi}^{*}$ of

$$
E^{\prime}(\varphi)=\{\tau \in E:\langle\tau, \alpha\rangle \neq 0 \forall \alpha \in \varphi\} .
$$

Suppose first that $\Phi$ contains no irreducible factors of type $A_{2 k}$. Since $\lambda$ is a discrete series or limit of discrete series parameter for $G_{\varphi}$ and $\lambda$ is in the closure of $E_{\varphi}^{*}, \lambda$ and $E^{*}$ also determine a discrete series or limit of discrete series character $\Theta_{\lambda}^{\varphi}$ for $G_{\varphi}$.

Now suppose that $\Phi$ contains irreducible factors of type $A_{2 k}, k \geq 1$. Let $\omega$ denote the set of all $X \in \underline{g}$ such that $|\operatorname{Im} \lambda|<\pi$ for every eigenvalue $\lambda$ of $a d X$. Define 
$\Omega=\exp (\omega) \subset G$. Then $\omega$ is an invariant neighborhood of the identity in $g$ and $\Omega$ is an invariant neighborhood of the identity in $G$. Let $\Omega^{\prime}=\Omega \cap G^{\prime}$. Now for any $\varphi \in \mathcal{T}(\Phi)$, we let $\Omega_{\varphi}^{\prime}$ denote the union of all good regular $G_{\varphi}$-orbits which map into $\Omega^{\prime}$ via the orbit map $F_{\varphi}$. If $\Theta$ is any class function on $\Omega_{\varphi}^{\prime}$, we can define a class function on $\Omega^{\prime}$ as in (6.1) by

$$
\left(\operatorname{Lift}_{\varphi}^{G} \Theta\right)(g)=\sum_{x \in X_{\varphi}(g)} D_{\varphi}^{G}(x) \Theta(x), \quad g \in \Omega^{\prime} .
$$

Note that by definition, for any $g \in \Omega^{\prime}, X_{\varphi}(g) \subset \Omega_{\varphi}^{\prime}$.

We will define a class function $\Theta_{\lambda}^{\varphi}$ on $\Omega_{\varphi}^{\prime}$. The only obstruction to defining $\Theta_{\lambda}^{\varphi}$ on $G_{\varphi}^{\prime}$ is that $\xi_{\lambda-\rho_{\varphi}}$ is not well-defined in the case that $e^{\rho\left(\Phi^{+}, \varphi\right)}$ is not well-defined on $B$. Let $E_{\Phi}$ denote the subspace of $E=i \underline{b}^{*}$ spanned by $\Phi$.

Lemma 6.2. Let $S \in \mathcal{S} O(\Phi)$, and $h \in H_{S} \cap \Omega^{\prime}$. Then there is $H \in \underline{h}_{S} \cap \omega$ such that $h=\exp H$. For all $\tau \in E_{\Phi}$,

$$
h \mapsto \xi_{\tau}(h)=e^{\tau\left(c_{S}^{-1} H\right)}
$$

gives a well defined function on $H_{S} \cap \Omega^{\prime}$. Finally, if $\left(w, S^{\prime}\right) \in \Lambda(h)$, then

$$
\xi_{\tau}\left(\left(w, S^{\prime}\right) \cdot h\right)=\xi_{w^{-1} \tau}(h) .
$$

Proof. Since $h \in \Omega$ there is $H \in \omega$ such that $h=\exp H$. Then $H \in C_{\underline{g}}(h)=\underline{h}_{S}$, so that $H \in \underline{h}_{S} \cap \omega$. Now suppose that $H, H^{\prime} \in \underline{h}_{S} \cap \omega$ such that $h \stackrel{\underline{g}}{=} \exp H=$ $\exp H^{\prime}$. For any $\alpha \in \Phi, \alpha\left(c_{S}^{-1} H-c_{S}^{-1} H^{\prime}\right) \in 2 \pi i \mathbf{Z}$. But $\left|\operatorname{Im} \alpha\left(c_{S}^{-1} H-c_{S}^{-1} H^{\prime}\right)\right| \leq$ $\left|\operatorname{Im} c_{S} \alpha(H)\right|+\left|\operatorname{Im} c_{S} \alpha\left(H^{\prime}\right)\right|<2 \pi$ because $H, H^{\prime} \in \omega$. Thus $\alpha\left(c_{S}^{-1} H-c_{S}^{-1} H^{\prime}\right)=$ 0 for all $\alpha \in \Phi$ so that $\tau\left(c_{S}^{-1} H\right)=\tau\left(c_{S}^{-1} H^{\prime}\right)$ for all $\tau \in E_{\Phi}$. Finally, if $h=$ $\exp H, H \in \underline{h}_{S} \cap \omega$, then for $\left(w, S^{\prime}\right) \in \Lambda(h),\left(w, S^{\prime}\right) \cdot h=\exp c_{S^{\prime}} w c_{S}^{-1} H$ where $c_{S^{\prime}} w c_{S}^{-1} H \in \underline{h}_{S^{\prime}} \cap \omega$. Thus by definition,

$$
\xi_{\tau}\left(\left(w, S^{\prime}\right) \cdot h\right)=e^{\tau\left(w c_{S}^{-1} H\right)}=e^{w^{-1} \tau\left(c_{S}^{-1} H\right)}=\xi_{w^{-1} \tau}(h) .
$$

Let $S \in \mathcal{S} O(\Phi, \varphi), h^{\prime} \in H_{\varphi, S} \cap \Omega_{\varphi}^{\prime}$, and let $h=f_{S}(h) \in H_{S} \cap \Omega^{\prime}$. Let $\Phi^{+}$be a choice of positive roots for $\Phi$. Then $\rho\left(\Phi^{+}, \varphi\right) \in E_{\Phi}$, so using Lemma 6.2 we can define

$$
\xi_{\rho\left(\Phi^{+}, \varphi\right)}\left(h^{\prime}\right)=\xi_{\rho\left(\Phi^{+}, \varphi\right)}(h), \quad \xi_{\lambda-\rho_{\varphi}}\left(h^{\prime}\right)=\xi_{\lambda-\rho_{\varphi}}(h)=\xi_{\lambda-\rho}(h) \xi_{\rho\left(\Phi^{+}, \varphi\right)}(h) .
$$

Now we can define $\Theta_{\lambda}^{\varphi}$ on $\Omega_{\varphi}^{\prime}$ using the formula in Theorem 3.3 applied to the group $G_{\varphi}$ with the discrete series constants corresponding to $E_{\varphi}^{*}$. (See Lemma 6.5 for details.) It corresponds to a discrete series character on a two-fold cover of $G_{\varphi}$.

In the case that $\Phi$ contains no irreducible factors of type $A_{2 n}, n \geq 1$, we define $\Omega=G, \Omega^{\prime}=G^{\prime}$, and $\Omega_{\varphi}^{\prime}=G_{\varphi}^{\prime}$. This will allow us to handle the two cases simultaneously from now on.

Let $\Phi^{+}$be a choice of positive roots for $\Phi$ and let $\varphi^{+}=\varphi \cap \Phi^{+}$. Recall that

$$
q_{G}=\frac{1}{2} \operatorname{dim}(G / K), \quad \epsilon_{G}\left(\Phi^{+}: E^{*}\right)=\epsilon_{G}\left(E^{*}\right)=\operatorname{sign} \prod_{\alpha \in \Phi^{+}}\langle\alpha, \tau\rangle, \quad \tau \in E^{*},
$$

and let

$$
q_{\varphi}=\frac{1}{2} \operatorname{dim}\left(G_{\varphi} / K_{\varphi}\right), \quad \epsilon_{\varphi}\left(\varphi^{+}: E^{*}\right)=\epsilon_{\varphi}\left(E^{*}\right)=\operatorname{sign} \prod_{\alpha \in \varphi^{+}}\langle\alpha, \tau\rangle, \quad \tau \in E^{*} .
$$


Define

$$
\epsilon_{\varphi}^{G}\left(E^{*}\right)=\epsilon_{\varphi}^{G}\left(\Phi^{+}: E^{*}\right)=\epsilon\left(\varphi: \Phi^{+}\right)(-1)^{q_{G}}(-1)^{q_{\varphi}} \epsilon_{\varphi}\left(\varphi^{+}: E^{*}\right) \epsilon_{G}\left(\Phi^{+}: E^{*}\right) .
$$

Lemma 6.3. $\epsilon_{\varphi}^{G}\left(E^{*}\right)$ is independent of the choice $\Phi^{+}$of positive roots.

Proof. Fix one choice $\Phi^{+}$of positive roots and write $\varphi^{+}=\varphi \cap \Phi^{+}$as above. Let $w \in W(\Phi)$ so that $w \Phi^{+}$is another choice of positive roots. Then there is a unique $u \in W(\varphi)$ so that $w \Phi^{+} \cap \varphi=u \varphi^{+}$. Further, $w^{-1} u \varphi^{+} \subset \Phi^{+}$so that $w^{-1} u \in W\left(\varphi: \Phi^{+}\right)$. Thus there is $v \in W\left(\varphi: \Phi^{+}\right)$so that $w=u v^{-1}$. Now by Lemma 5.2,

$$
\epsilon\left(\varphi: w \Phi^{+}\right)=\epsilon\left(v u^{-1} \varphi: \Phi^{+}\right)=\epsilon\left(v \varphi: \Phi^{+}\right)=\operatorname{det} v \epsilon\left(\varphi: \Phi^{+}\right) .
$$

But

$$
\epsilon_{G}\left(w \Phi^{+}: E^{*}\right)=\operatorname{det} w \epsilon_{G}\left(\Phi^{+}: E^{*}\right)
$$

and

$$
\epsilon_{\varphi}\left(u \varphi^{+}: E^{*}\right)=\operatorname{det} u \epsilon_{\varphi}\left(\varphi^{+}: E^{*}\right)
$$

For $S \in \mathcal{S O}(\Phi), h=t a \in H_{S}^{1}$, define

$$
c(h)=c\left(\Phi_{t}\right) c(K, h) .
$$

Here $\left.c(K, h)=\left[W_{0}(h) / W\left(\Phi_{K, t}\right)\right)\right]$ is the constant appearing in the discrete series character formula of Theorem 3.3, and $c\left(\Phi_{t}\right)$ is the constant occurring in Theorem 3.4. Note that $c(h)$ depends only on the connected component of $h$ in $H_{S}^{1}$. Further, if $S, S^{\prime} \in \mathcal{S} O(\Phi), h=t a \in H_{S}^{1} \cap G^{\prime}, h^{\prime}=t^{\prime} a^{\prime} \in H_{S^{\prime}} \cap G^{\prime}$ such that $\mathcal{O}_{G}(h)=\mathcal{O}_{G}\left(h^{\prime}\right)$, then $\Phi_{t}$ and $\Phi_{t^{\prime}}$ are conjugate via $W\left(\Phi_{K}\right)$, so that $c(h)=c\left(h^{\prime}\right)$. Thus if $g \in G^{\prime}$ and $S \in \mathcal{S O}(\Phi), h \in H_{S}^{1}$, such that $\mathcal{O}_{G}(g)=\mathcal{O}_{G}(h)$, we can define $c(g)=c(h)$, and this is independent of the choice of $S$ and $h$.

Recall that in $\S 4$, for any $S \in \mathcal{S O}(\Phi)$ we defined $H_{S}^{\prime \prime}$ to be the set of all $h \in H_{S}^{1} \cap G^{\prime}$ such that if $(w, S) \in \Lambda(h)$ and $(w, S) \cdot h=h$, then $w=1$. Let $G^{\prime \prime}$ be the set of all $g \in G$ such that there are $S \in \mathcal{S O}(\Phi), h \in H_{S}^{\prime \prime}$, such that $\mathcal{O}_{G}(g)=\mathcal{O}_{G}(h)$. Using Theorem 2.5 and Lemma 2.6 it is easy to see that this definition is also independent of the choice of $S$ and $h$.

Theorem 6.4. Let $\lambda$ be any discrete series or limit of discrete series parameter for $G$. Then for all $g \in \Omega \cap G^{\prime \prime}$,

$$
\Theta_{\lambda}(g)=c(g) \sum_{\varphi \in \mathcal{T}(\Phi)} \epsilon_{\varphi}^{G}\left(E^{*}\right)\left(\operatorname{Lift}_{\varphi}^{G} \Theta_{\lambda}^{\varphi}\right)(g)
$$

The remainder of this section is devoted to the proof of Theorem 6.4. We will reduce the proof to a technical result, Lemma 6.8 , which will be proven in $\S 7$.

Fix a set of positive roots $\Phi^{+}$and let $\varphi^{+}=\Phi^{+} \cap \varphi, \varphi \in \mathcal{T}(\Phi)$. Define

$$
\rho_{G}=\rho\left(\Phi^{+}\right)=\frac{1}{2} \sum_{\alpha \in \Phi^{+}} \alpha, \rho_{\varphi}=\rho\left(\varphi^{+}\right)=\frac{1}{2} \sum_{\alpha \in \varphi^{+}} \alpha, \rho\left(\Phi^{+}, \varphi\right)=\rho\left(\Phi^{+}\right)-\rho\left(\varphi^{+}\right) \text {. }
$$

Now let $S \in \mathcal{S} O(\Phi)$ with corresponding Cayley transform $c_{S}$. When $\Phi$ contains no irreducible factors of type $A_{2 n}, n \geq 1, \rho\left(\Phi^{+}, \varphi\right)$ is in the root lattice of $\Phi$ by Theorem 5.5. Thus for $h=t a \in H_{S}^{1}$, as in $\S 3$ we can define

$$
\xi_{\rho\left(\Phi^{+}, \varphi\right)}(h)=e^{\rho\left(\Phi^{+}, \varphi\right)}(t) \exp \left(\rho\left(\Phi^{+}, \varphi\right)\left(c_{S}^{-1} \log a\right)\right) .
$$


Otherwise, we define $\xi_{\rho\left(\Phi^{+}, \varphi\right)}(h), h \in H_{S}^{1} \cap \Omega^{\prime}$, using Lemma 6.2. Now for $h \in$ $H_{S}^{1} \cap \Omega^{\prime}$, we can define

$$
\Delta_{\varphi}^{G}\left(\Phi^{+}: h\right)=\prod_{\alpha \in c_{S}\left(\Phi^{+} \backslash \varphi^{+}\right)}\left(1-e^{-\alpha}(h)\right) \xi_{-\rho\left(\Phi^{+}, \varphi\right)}(h) .
$$

We also define

$$
\delta\left(\Phi^{+}, \varphi, h\right)=\left|\Delta_{\varphi}^{G}\left(\Phi^{+}: h\right)\right| \Delta_{\varphi}^{G}\left(\Phi^{+}: h\right)^{-1} .
$$

Suppose that $S \in \mathcal{S} O(\Phi, \varphi)$. As in $\S 3$, define

$$
\begin{array}{cc}
\Delta_{G}^{\prime}\left(\Phi^{+}: h\right)=\Delta_{G}^{\prime}(h)=\prod_{\alpha \in c_{S} \Phi^{+}}\left(1-e^{-\alpha}(h)\right), \quad h \in H_{S} ; \\
\Delta_{\varphi}^{\prime}\left(\varphi^{+}: h^{\prime}\right)=\Delta_{\varphi}^{\prime}\left(h^{\prime}\right)=\prod_{\alpha \in c_{S} \varphi^{+}}\left(1-e^{-\alpha}\left(h^{\prime}\right)\right), \quad h^{\prime} \in H_{\varphi, S} .
\end{array}
$$

Then for $h^{\prime} \in H_{\varphi, S}$ such that $f_{S}\left(h^{\prime}\right)=h \in \Omega^{\prime}$, we have

$$
\Delta_{\varphi}^{G}\left(\Phi^{+}: h\right)=\Delta_{G}^{\prime}\left(\Phi^{+}: h\right)^{-1} \Delta_{\varphi}^{\prime}\left(\varphi^{+}: h^{\prime}\right) \xi_{-\rho\left(\Phi^{+}, \varphi\right)}(h),
$$

and it is easy to see that

$$
D_{\varphi}^{G}\left(h^{\prime}\right)=\left|\Delta_{\varphi}^{G}\left(\Phi^{+}: h\right)\right|=\delta\left(\Phi^{+}, \varphi, h\right) \Delta_{\varphi}^{G}\left(\Phi^{+}: h\right) .
$$

Fix a choice $\Phi^{+}$of positive roots, and for $\varphi \in \mathcal{T}(\Phi)$, let $\varphi^{+}=\Phi^{+} \cap \varphi$. We use these choices of positive roots to define $\Delta_{G}^{\prime}, \Delta_{\varphi}^{\prime}, \rho_{G}, \rho_{\varphi}, \epsilon_{G}\left(E^{*}\right)$, and $\epsilon_{\varphi}\left(E^{*}\right)$ for all $\varphi \in \mathcal{T}(\Phi)$.

Fix $\varphi \in \mathcal{T}(\Phi), S \in \mathcal{S} O(\Phi, \varphi)$. We use the notation of $\S 3$ for $G_{\varphi}$. Then $\Theta_{\lambda}^{\varphi}$ is given on $H_{\varphi, S} \cap \Omega_{\varphi}^{\prime}$ by the following formula.

Lemma 6.5. Let $h^{\prime}=t^{\prime} a^{\prime} \in H_{\varphi, S} \cap \Omega_{\varphi}^{\prime}$. Then

$$
\begin{gathered}
(-1)^{q_{\varphi}} \epsilon_{\varphi}\left(E^{*}\right) \Theta_{\lambda}^{\varphi}\left(h^{\prime}\right) \\
=\sum_{w \in W\left(\varphi_{K}, h^{\prime}\right)} \Delta_{\varphi}^{\prime}\left(w \cdot h^{\prime}\right)^{-1} \xi_{\lambda-\rho_{\varphi}}\left(w \cdot h^{\prime}\right) c\left(1: E_{\varphi}^{*}: \varphi_{R}^{+}\left(w \cdot h^{\prime}\right)\right) .
\end{gathered}
$$

Proof. In the case that $\lambda$ is a discrete series or limit of discrete series parameter for $G_{\varphi}$, this follows directly from Theorem 3.3 since by Lemma 4.3(ii), $W\left(\varphi_{K}\right) \cap$ $W\left(\varphi_{t^{\prime}}\right)=W\left(\varphi_{K, t^{\prime}}\right)$, so that the constant $c\left(K_{\varphi}, h^{\prime}\right)=1$. Otherwise, this formula can be taken as the definition of $\Theta_{\lambda}^{\varphi}$.

For $h \in H_{S}^{1} \cap \Omega^{\prime}$ with $f_{S}^{-1}(h)=h^{\prime} \in H_{\varphi, S}$, write $W\left(\varphi_{K}, h\right)=W\left(\varphi_{K}, h^{\prime}\right)$. We also write $\varphi_{R}^{+}(h)=\varphi_{R}^{+}\left(h^{\prime}\right)$ and $c\left(1: E^{*}: \varphi_{R}^{+}(h)\right)=c\left(1: E_{\varphi}^{*}: \varphi_{R}^{+}\left(h^{\prime}\right)\right)$. For $w \in W\left(\Phi_{K}, \varphi, h\right)$, we will write

$$
\delta\left(\Phi^{+}, \varphi, w \cdot h\right)=\delta\left(\Phi^{+}, \varphi,\left(w, S^{\prime}\right) \cdot h\right)
$$

where $S^{\prime} \in \mathcal{S} O(\Phi, \varphi)$ such that $\left(w, S^{\prime}\right) \in \Lambda(h)$. It is easy to see that it is independent of the choice of $S^{\prime}$.

Lemma 6.6. Let $h \in H_{S}^{1} \cap \Omega^{\prime}, S \in \mathcal{S} O(\Phi, \varphi)$. Then

$$
\begin{aligned}
\epsilon_{\varphi}^{G}\left(E^{*}\right) & D_{\varphi}^{G}\left(f_{S}^{-1}(h)\right) \Theta_{\lambda}^{\varphi}\left(f_{S}^{-1}(h)\right)=\epsilon\left(\varphi: \Phi^{+}\right) \epsilon_{G}\left(E^{*}\right)(-1)^{q_{G}} \\
\quad \times & \sum_{w \in W\left(\varphi_{K}, h\right)} \Delta_{G}^{\prime}(w \cdot h)^{-1} \xi_{\lambda-\rho_{G}}(w \cdot h) \delta\left(\Phi^{+}, \varphi, w \cdot h\right) c\left(1: E^{*}: \varphi_{R}^{+}(w \cdot h)\right) .
\end{aligned}
$$


Proof. Let $f_{S}^{-1}(h)=h^{\prime}=t^{\prime} a^{\prime} \in H_{\varphi, S}$. Then $D_{\varphi}^{G}\left(h^{\prime}\right)=D_{\varphi}^{G}\left(w \cdot h^{\prime}\right)$ for all $w \in$ $W\left(\varphi_{K}, h^{\prime}\right)$. Thus using (6.6), Lemma 6.5, and the definition of $\epsilon_{\varphi}^{G}\left(E^{*}\right)$,

$$
\begin{aligned}
\epsilon_{\varphi}^{G}\left(E^{*}\right) & D_{\varphi}^{G}\left(h^{\prime}\right) \Theta_{\lambda}^{\varphi}\left(h^{\prime}\right)=\epsilon\left(\varphi: \Phi^{+}\right) \epsilon_{G}\left(E^{*}\right)(-1)^{q_{G}} \\
\quad & \times \sum_{w \in W\left(\varphi_{K}, h^{\prime}\right)} D_{\varphi}^{G}\left(w \cdot h^{\prime}\right) \Delta_{\varphi}^{\prime}\left(w \cdot h^{\prime}\right)^{-1} \xi_{\lambda-\rho_{\varphi}}\left(w \cdot h^{\prime}\right) c\left(1: E^{*}: \varphi_{R}^{+}\left(w \cdot h^{\prime}\right)\right) \\
= & \epsilon\left(\varphi: \Phi^{+}\right) \epsilon_{G}\left(E^{*}\right)(-1)^{q_{G}} \\
\quad & \times \sum_{w \in W\left(\varphi_{K}, h^{\prime}\right)} \Delta_{G}^{\prime}(w \cdot h)^{-1} \xi_{\lambda-\rho_{G}}\left(w \cdot h^{\prime}\right) \delta\left(\Phi^{+}, \varphi, w \cdot h\right) c\left(1: E^{*}: \varphi_{R}^{+}\left(w \cdot h^{\prime}\right)\right) .
\end{aligned}
$$

But

$$
\xi_{\lambda-\rho_{G}}\left(w \cdot h^{\prime}\right)=\xi_{\lambda-\rho_{G}}(w \cdot h)
$$

Lemma 6.7. Let $\varphi \in \mathcal{T}(\Phi), S \in \mathcal{S} O(\Phi), h \in H_{S}^{\prime \prime} \cap \Omega$. Then

$$
\begin{aligned}
\epsilon_{\varphi}^{G}\left(E^{*}\right) & \left(\operatorname{Lift}_{\varphi}^{G} \Theta_{\lambda}^{\varphi}\right)(h) \\
= & \epsilon_{G}\left(E^{*}\right)(-1)^{q_{G}} \sum_{w \in W\left(\Phi_{K}, \varphi, h\right)} \epsilon\left(\varphi: \Phi^{+}\right) \delta\left(\Phi^{+}, \varphi, w \cdot h\right) \\
& \times \Delta_{G}^{\prime}(w \cdot h)^{-1} \xi_{\lambda-\rho_{G}}(w \cdot h) c\left(1: E^{*}: \varphi_{R}^{+}(w \cdot h)\right) .
\end{aligned}
$$

Proof. By Theorem 4.13, we can take

$$
X_{\varphi}(h)=\left\{f_{S_{i}}^{-1}\left(\left(w_{i}, S_{i}\right) \cdot h\right): 1 \leq i \leq p\right\}
$$

where the $w_{i}=\left[v_{i}, s_{i}\right], 1 \leq i \leq p$, are a complete set of representatives of the equivalence classes in $W\left(\Phi_{K}, \varphi, h\right)$ for the equivalence relation $\sim_{\varphi, h}$, and $S_{i} \in$ $\mathcal{S} O(\Phi, \varphi), 1 \leq i \leq p$. Write

$$
h_{i}^{\prime}=f_{S_{i}}^{-1}\left(\left(w_{i}, S_{i}\right) \cdot h\right), \quad h_{i}=\left(w_{i}, S_{i}\right) \cdot h, \quad 1 \leq i \leq p .
$$

Then we have

$$
\epsilon_{\varphi}^{G}\left(E^{*}\right)\left(\operatorname{Lift}_{\varphi}^{G} \Theta_{\lambda}^{\varphi}\right)(h)=\epsilon_{\varphi}^{G}\left(E^{*}\right) \sum_{1 \leq i \leq p} D_{\varphi}^{G}\left(h_{i}^{\prime}\right) \Theta_{\lambda}^{\varphi}\left(h_{i}^{\prime}\right) .
$$

Fix $1 \leq i \leq p$. Then using Lemma 6.6,

$$
\begin{aligned}
& \epsilon_{\varphi}^{G}\left(E^{*}\right) D_{\varphi}^{G}\left(h_{i}^{\prime}\right) \Theta_{\lambda}^{\varphi}\left(h_{i}^{\prime}\right)=\epsilon\left(\varphi: \Phi^{+}\right) \epsilon_{G}\left(E^{*}\right)(-1)^{q_{G}} \\
& \quad \times \sum_{w \in W\left(\varphi_{K}, h_{i}\right)} \Delta_{G}^{\prime}\left(w \cdot h_{i}\right)^{-1} \xi_{\lambda-\rho_{G}}\left(w \cdot h_{i}\right) \delta\left(\Phi^{+}, \varphi, w \cdot h_{i}\right) c\left(1: E^{*}: \varphi_{R}^{+}\left(w \cdot h_{i}\right)\right) .
\end{aligned}
$$

Let $w \in W\left(\varphi_{K}, h_{i}\right)$. Then there are $v \in W\left(\varphi_{K}\right), u \in W\left(\varphi_{v_{i} t}\right)$ such that $w=v u$, and $v S_{i} \in \mathcal{S} O(\Phi, \varphi)$. Then $\left(w, v S_{i}\right) \in \Lambda\left(\varphi, h_{i}^{\prime}\right) \subset \Lambda\left(h_{i}\right)$ and $\left(w, v S_{i}\right) \cdot h_{i}=\left(w, v S_{i}\right)$. $\left(w_{i}, S_{i}\right) \cdot h=\left(w w_{i}, v S_{i}\right) \cdot h$ where $w w_{i} \in W\left(\Phi_{K}, \varphi, h\right)$ by Lemma 4.11 . Thus we can write

$$
\begin{gathered}
\epsilon_{\varphi}^{G}\left(E^{*}\right) D_{\varphi}^{G}\left(h_{i}^{\prime}\right) \Theta_{\lambda}^{\varphi}\left(h_{i}^{\prime}\right) \\
=\epsilon_{G}\left(E^{*}\right)(-1)^{q_{G}} \sum_{w \in W\left(\varphi_{K}, h_{i}\right)} \delta\left(\Phi^{+}, \varphi, w w_{i} \cdot h\right) \epsilon\left(\varphi: \Phi^{+}\right) \\
\times \Delta_{G}^{\prime}\left(w w_{i} \cdot h\right)^{-1} \xi_{\lambda-\rho_{G}}\left(w w_{i} \cdot h\right) c\left(1: E^{*}: \varphi_{R}^{+}\left(w w_{i} \cdot h\right)\right) .
\end{gathered}
$$


By Lemma 4.12 (ii), for each $\sigma \in W\left(\Phi_{K}, \varphi, h\right)$ there are unique $1 \leq i \leq p$ and $w \in W\left(\varphi_{K}, h_{i}\right)$ such that $\sigma=w w_{i}$. Thus

$$
\begin{aligned}
\epsilon_{\varphi}^{G}\left(E^{*}\right) & \left(\operatorname{Lift}_{\varphi}^{G} \Theta_{\lambda}^{\varphi}\right)(h) \\
= & \epsilon_{G}\left(E^{*}\right)(-1)^{q_{G}} \sum_{w \in W\left(\Phi_{K}, \varphi, h\right)} \delta\left(\Phi^{+}, \varphi, w \cdot h\right) \epsilon\left(\varphi: \Phi^{+}\right) \\
& \times \Delta_{G}^{\prime}(w \cdot h)^{-1} \xi_{\lambda-\rho_{G}}(w \cdot h) c\left(1: E^{*}: \varphi_{R}^{+}(w \cdot h)\right) .
\end{aligned}
$$

We will prove the following technical lemma in $\S 7$. Define $\mathcal{T}_{\text {aug }}\left(\Phi_{t}\right)$ as in $\S 5$. For $\psi \in \mathcal{T}_{\text {aug }}\left(\Phi_{t}\right)$, let

$$
\mathcal{T}(\Phi, \psi)=\left\{\varphi \in \mathcal{T}(\Phi): \varphi \cap \Phi_{t}=\psi\right\} .
$$

Lemma 6.8. Let $S \in \mathcal{S} O(\Phi), h=t a \in H_{S}^{1} \cap \Omega^{\prime}$. Let $\Phi^{+}$be any choice of positive roots for $\Phi$ and let $\psi \in \mathcal{T}_{\text {aug }}\left(\Phi_{t}\right)$. Then

$$
\sum_{\varphi \in \mathcal{T}(\Phi, \psi)} \epsilon\left(\varphi: \Phi^{+}\right) \delta\left(\Phi^{+}, \varphi, h\right)= \begin{cases}\epsilon\left(\psi: \Phi_{R}^{+}(h)\right), & \text { if } \psi \in \mathcal{T}\left(\Phi_{t}\right) ; \\ 0, & \text { otherwise. }\end{cases}
$$

Assuming Lemma 6.8, we can complete the proof of Theorem 6.4 as follows.

Proof of Theorem 6.4. Since both sides of the equation are class functions on $G^{\prime \prime} \cap$ $\Omega$, it suffices to prove the equation for every $h \in H_{S}^{\prime \prime} \cap \Omega, S \in \mathcal{S} O(\Phi)$.

Fix $h \in H_{S}^{\prime \prime} \cap \Omega, S \in \mathcal{S} O(\Phi)$. Recall that $c(h)=c\left(\Phi_{t}\right) c(K, h)$. Thus by Lemma 6.7 we have

$$
\begin{gathered}
c(h) \sum_{\varphi \in \mathcal{T}(\Phi)} \epsilon_{\varphi}^{G}\left(E^{*}\right)\left(\operatorname{Lift}_{\varphi}^{G} \Theta_{\lambda}^{\varphi}\right)(h) \\
=\epsilon_{G}\left(E^{*}\right)(-1)^{q_{G}} c(K, h) \sum_{w \in W\left(\Phi_{K}, h\right)} \Delta_{G}^{\prime}(w \cdot h)^{-1} \xi_{\lambda-\rho_{G}}(w \cdot h) \\
\times c\left(\Phi_{t}\right) \sum_{\varphi \in \mathcal{T}(\Phi, h, w)} \delta\left(\Phi^{+}, \varphi, w \cdot h\right) \epsilon\left(\varphi: \Phi^{+}\right) c\left(1: E^{*}: \varphi_{R}^{+}(w \cdot h)\right),
\end{gathered}
$$

where $\mathcal{T}(\Phi, h, w)$ denotes the set of all $\varphi \in \mathcal{T}(\Phi)$ such that $w \in W\left(\Phi_{K}, \varphi, h\right)$. Using the formula for $\Theta_{\lambda}(h)$ in Theorem 3.3, we see that it suffices to prove that for all $w \in W\left(\Phi_{K}, h\right)$,

$$
\begin{aligned}
c\left(\Phi_{t}\right) & \sum_{\varphi \in \mathcal{T}(\Phi, h, w)} \delta\left(\Phi^{+}, \varphi, w \cdot h\right) \epsilon\left(\varphi: \Phi^{+}\right) c\left(1: E^{*}: \varphi_{R}^{+}(w \cdot h)\right) \\
= & c\left(1: E^{*}: \Phi_{R}^{+}(w \cdot h)\right) .
\end{aligned}
$$

Define $\mathcal{T}(\Phi, h)=\left\{\varphi \in \mathcal{T}(\Phi): \exists S^{\prime} \in \mathcal{S} O(\Phi, \varphi)\right.$ with $\left.\left(1, S^{\prime}\right) \in \Lambda(h)\right\}$. Let $\left(w, S^{\prime}\right) \in \Lambda(h)$. Then it is easy to see using Lemma 2.6 that

$$
\mathcal{T}(\Phi, h, w)=\mathcal{T}\left(\Phi,\left(w, S^{\prime}\right) \cdot h\right) .
$$

For $w=[v, s] \in W\left(\Phi_{K}, h\right), c\left(\Phi_{t}\right)=c\left(\Phi_{v t}\right)$. Thus,

$$
c\left(\Phi_{t}\right) \sum_{\varphi \in \mathcal{T}(\Phi, h, w)} \delta\left(\Phi^{+}, \varphi, w \cdot h\right) \epsilon\left(\varphi: \Phi^{+}\right) c\left(1: E^{*}: \varphi_{R}^{+}(w \cdot h)\right)
$$


depends only on $\left(w, S^{\prime}\right) \cdot h$, not on $w$ and $h$ separately, and so we see that it suffices to prove that for all $S \in \mathcal{S} O(\Phi), h \in H_{S}^{1} \cap \Omega^{\prime}$,

$$
c\left(\Phi_{t}\right) \sum_{\varphi \in \mathcal{T}(\Phi, h)} \delta\left(\Phi^{+}, \varphi, h\right) \epsilon\left(\varphi: \Phi^{+}\right) c\left(1: E^{*}: \varphi_{R}^{+}(h)\right)=c\left(1: E^{*}: \Phi_{R}^{+}(h)\right) .
$$

Let $\varphi \in \mathcal{T}(\Phi, h)$, and let $\psi=\varphi \cap \Phi_{t}$. Then $\varphi_{R}^{+}(h)=\psi \cap \Phi_{R}^{+}(h)$ depends only on $\psi$. Further, by definition there is $S^{\prime} \in \mathcal{S O}(\Phi, \varphi)$ such that $\left(1, S^{\prime}\right) \in \Lambda(h)$. Thus $S^{\prime} \subset \Phi_{t}$ and spans $\Phi_{t}$. Now $S^{\prime} \subset \psi$ and spans $\psi$, so that rank $\psi=\operatorname{rank} \Phi_{t}$. Clearly every irreducible factor of $\psi$ is of type $A_{1}$ or $B_{2}$. Thus $\psi \in \mathcal{T}_{\text {aug }}\left(\Phi_{t}\right)$. Further, since $S^{\prime} \subset \psi$, we have $\psi \in \mathcal{T}_{n}\left(\Phi_{t}\right)$ if and only if $\psi \in \mathcal{T}\left(\Phi_{t}\right)$.

Thus we can write

$$
\begin{gathered}
c\left(\Phi_{t}\right) \sum_{\varphi \in \mathcal{T}(\Phi, h)} \epsilon\left(\varphi: \Phi^{+}\right) \delta\left(\Phi^{+}, \varphi, h\right) c\left(1: E^{*}: \varphi_{R}^{+}(h)\right) \\
=c\left(\Phi_{t}\right) \sum_{\psi \in \mathcal{T}_{\text {aug }}\left(\Phi_{t}\right)} c\left(1: E^{*}: \psi \cap \Phi_{R}^{+}(h)\right) \sum_{\varphi \in \mathcal{T}(\Phi, h, \psi)} \epsilon\left(\varphi: \Phi^{+}\right) \delta\left(\Phi^{+}, \varphi, h\right),
\end{gathered}
$$

where for $\psi \in \mathcal{T}_{\text {aug }}\left(\Phi_{t}\right), \mathcal{T}(\Phi, h, \psi)$ is the set of all $\varphi \in \mathcal{T}(\Phi, h)$ such that $\varphi \cap \Phi_{t}=\psi$.

Let $\psi \in \mathcal{T}_{\text {aug }}\left(\Phi_{t}\right)$. If there is no $S^{\prime} \in \mathcal{S} O(\Phi)$ such that $\left(1, S^{\prime}\right) \in \Lambda(h)$ and $S^{\prime} \subset \psi$, then by the above $\mathcal{T}(\Phi, \psi, h)=\emptyset$. Suppose that there is $S^{\prime} \in \mathcal{S} O(\Phi)$ such that $\left(1, S^{\prime}\right) \in \Lambda(h)$ and $S^{\prime} \subset \psi$. Then for all $\varphi \in \mathcal{T}(\Phi, \psi), S^{\prime} \subset \varphi$ so that $\mathcal{T}(\Phi, \psi)=\mathcal{T}(\Phi, \psi, h)$. Now if $\psi \notin \mathcal{T}(\Phi)$

$$
\sum_{\varphi \in \mathcal{T}(\Phi, h, \psi)} \epsilon\left(\varphi: \Phi^{+}\right) \delta\left(\Phi^{+}, \varphi, h\right)=\sum_{\varphi \in \mathcal{T}(\Phi, \psi)} \epsilon\left(\varphi: \Phi^{+}\right) \delta\left(\Phi^{+}, \varphi, h\right)=0
$$

by Lemma 6.8 . If $\psi \in \mathcal{T}\left(\Phi_{t}\right)$, then $\psi \in \mathcal{T}_{n}\left(\Phi_{t}\right)$, and again using Lemma 6.8 ,

$$
\sum_{\varphi \in \mathcal{T}(\Phi, h, \psi)} \epsilon\left(\varphi: \Phi^{+}\right) \delta\left(\Phi^{+}, \varphi, h\right)=\sum_{\varphi \in \mathcal{T}(\Phi, \psi)} \epsilon\left(\varphi: \Phi^{+}\right) \delta\left(\Phi^{+}, \varphi, h\right)=\epsilon\left(\psi: \Phi_{R}^{+}(h)\right) .
$$

Thus by Theorem 3.4 we have

$$
\begin{gathered}
c\left(\Phi_{t}\right) \sum_{\varphi \in \mathcal{T}(\Phi, h)} \epsilon\left(\varphi: \Phi^{+}\right) \delta\left(\Phi^{+}, \varphi, h\right) c\left(1: E^{*}: \varphi_{R}^{+}(h)\right) \\
=c\left(\Phi_{t}\right) \sum_{\psi \in \mathcal{T}_{n}\left(\Phi_{t}\right)} \epsilon\left(\psi: \Phi_{R}^{+}(h)\right) c\left(1: E^{*}: \psi \cap \Phi_{R}^{+}(h)\right)=c\left(1: E^{*}: \Phi_{R}^{+}(h)\right) .
\end{gathered}
$$

\section{Proof of Lemma 6.8}

We keep the notation of $\S 6$. We may as well assume that $\Phi$ is irreducible. Throughout this section we fix $S \in \mathcal{S O}(\Phi)$ and $h=t a \in H_{S}^{1} \cap \Omega^{\prime}$. Further, we fix $\gamma \in T_{S} \cap B_{S}$ such that $h \in \gamma H_{S}^{0}$, and $H_{1} \in \underline{t}_{S}$ such that $\gamma^{-1} t=\exp \left(H_{1}\right)$. Let $H_{2}=c_{S}^{-1} \log a \in i \underline{b}_{S}$. Thus $h=\gamma \exp \left(c_{S} H\right)$ where $H=H_{1}+H_{2}$. In the case that $\Phi$ is of type $A_{2 n}, n \geq 1$, by Lemma 6.2 we can assume that $\gamma=1$, and $H_{1}+c_{S} H_{2} \in \omega$. Thus in this case for any $\tau \in E_{\Phi}$ we define $e^{\tau}(\gamma)=1$. For any $\alpha \in \Phi$, write

$$
\begin{gathered}
\Delta(\alpha)=e^{\frac{\alpha(H)}{2}}-e^{-\alpha}(\gamma) e^{-\frac{\alpha(H)}{2}} \\
\delta(\alpha)=\Delta(\alpha)|\Delta(\alpha)|^{-1} .
\end{gathered}
$$

The following lemma is an easy consequence of the definitions. 
Lemma 7.1. For any $\varphi \in \mathcal{T}(\Phi)$ and any choice $\Phi^{+}$of positive roots,

$$
\delta\left(\Phi^{+}, \varphi, h\right)=e^{\rho\left(\Phi^{+}, \varphi\right)}(\gamma) \prod_{\alpha \in \Phi^{+} \backslash\left(\varphi \cap \Phi^{+}\right)} \delta(\alpha) .
$$

Lemma 7.2. For any $\varphi \in \mathcal{T}(\Phi), \epsilon\left(\varphi: \Phi^{+}\right) \delta\left(\Phi^{+}, \varphi, h\right)$ is independent of the choice $\Phi^{+}$of positive roots.

Proof. Fix one choice $\Phi^{+}$of positive roots and write $\varphi^{+}=\varphi \cap \Phi^{+}$. Let $w \in W(\Phi)$ so that $w \Phi^{+}$is another choice of positive roots. Then as in Lemma 6.3 we can write $w=u v^{-1}$ where $u \in W(\varphi)$ with $w \Phi^{+} \cap \varphi=u \varphi^{+}$and $v \in W\left(\varphi: \Phi^{+}\right)$. Then as in Lemma 6.3,

$$
\epsilon\left(\varphi: w \Phi^{+}\right)=\operatorname{det} v \epsilon\left(\varphi: \Phi^{+}\right)
$$

By Lemma 7.1 we have

$$
\delta\left(\Phi^{+}, \varphi, h\right)=e^{\rho\left(\Phi^{+}\right)-\rho\left(\varphi^{+}\right)}(\gamma) \prod_{\alpha \in \Phi^{+} \backslash\left(\varphi^{+}\right)} \delta(\alpha) .
$$

It is easy to check from the definition that for any $\alpha \in \Phi$,

$$
\delta(-\alpha)=-e^{\alpha}(\gamma) \delta(\alpha)
$$

Thus we have

$$
\begin{gathered}
\prod_{\alpha \in w \Phi^{+}} \delta(\alpha)=\operatorname{det} w e^{\rho\left(\Phi^{+}\right)-\rho\left(w \Phi^{+}\right)}(\gamma) \prod_{\alpha \in \Phi^{+}} \delta(\alpha), \\
\prod_{\alpha \in u \varphi^{+}} \delta(\alpha)=\operatorname{det} u e^{\rho\left(\varphi^{+}\right)-\rho\left(u \varphi^{+}\right)}(\gamma) \prod_{\alpha \in \varphi^{+}} \delta(\alpha) .
\end{gathered}
$$

Thus

$$
\delta\left(w \Phi^{+}, \varphi, h\right)=\operatorname{det} v \delta\left(\Phi^{+}, \varphi, h\right)
$$

Let $\Phi_{S}=c_{S} \Phi=\Phi\left(\underline{g}_{\mathbf{C}}, \underline{h}_{S, \mathbf{C}}\right)$. Then we can define

$$
\begin{gathered}
\Phi_{R, S}=\left\{\alpha \in \Phi_{S}: \alpha(H) \in \mathbf{R} \forall H \in \underline{h}_{S}\right\} ; \\
\Phi_{I, S}=\left\{\alpha \in \Phi_{S}: \alpha(H) \in i \mathbf{R} \forall H \in \underline{h}_{S}\right\} ; \\
\Phi_{C P X, S}=\Phi_{S} \backslash\left(\Phi_{R, S} \cup \Phi_{I, S}\right) .
\end{gathered}
$$

As in $\S 4$ we let $\sigma$ be the involution of $\underline{g}_{\mathbf{C}}$ with fixed points $\underline{g}$. For each $\alpha \in \Phi_{S}$ we have $\alpha^{\sigma} \in \Phi_{S}$ defined by

$$
\alpha^{\sigma}(H)=\overline{\alpha(\sigma(H))}, \quad H \in \underline{h}_{S, C} .
$$

Then $\alpha^{\sigma}=\alpha$ if and only if $\alpha \in \Phi_{R, S}$ and $\alpha^{\sigma}=-\alpha$ if and only if $\alpha \in \Phi_{I, S}$.

Define

$$
\Phi_{R}=c_{S}^{-1} \Phi_{R, S}, \quad \Phi_{I}=c_{S}^{-1} \Phi_{I, S}, \quad \Phi_{C P X}=c_{S}^{-1} \Phi_{C P X, S} .
$$

For any $\alpha \in \Phi$ we will write $\alpha^{\sigma, S}=c_{S}^{-1}\left(c_{S} \alpha\right)^{\sigma}$. Let $\Phi^{+}$be a choice of positive roots for $\Phi$. We say that $\Phi_{C P X}^{+}=\Phi^{+} \cap \Phi_{C P X}$ is $\sigma$-stable if $\alpha \in \Phi_{C P X}^{+}$if and only if $\alpha^{\sigma, S} \in \Phi_{C P X}^{+}$.

Lemma 7.3. Let $\varphi \in \mathcal{T}(\Phi)$ such that $\varphi \cap \Phi_{t} \in \mathcal{T}_{\text {aug }}\left(\Phi_{t}\right)$. Then for any $\alpha \in \Phi$, $\alpha \in \varphi$ if and only if $\alpha^{\sigma, S} \in \varphi$. 
Proof. Suppose $\alpha \in \Phi, H_{1} \in \underline{t}_{S}, H_{2} \in \underline{b}_{S}$. Then $\alpha^{\sigma, S}\left(H_{1}+H_{2}\right)=\alpha\left(-H_{1}+H_{2}\right)$. Since $\varphi \cap \Phi_{t} \in \mathcal{T}_{\text {aug }}\left(\Phi_{t}\right)$, there is an orthogonal set $S^{\prime} \subset \varphi \cap \Phi_{t}$ such that $\left[S^{\prime}\right]=$ rank $\Phi_{t}$. Let $w_{S^{\prime}}$ denote the product of the reflections corresponding to the roots in $S^{\prime}$. Then $w_{S^{\prime}} \in W(\varphi)$ and $w_{S^{\prime}}\left(H_{1}+H_{2}\right)=H_{1}-H_{2}$ for all $H_{1} \in \underline{t}_{S}, H_{2} \in \underline{b}_{S}$. Thus for all $\alpha \in \Phi, \alpha^{\sigma, S}=-w_{S^{\prime}} \alpha$. Thus $\alpha \in \varphi$ if and only if $\alpha^{\sigma, S} \in \varphi$.

Note that

$$
\Phi_{t}=\left\{\alpha \in \Phi_{R}: e^{\alpha}(\gamma)=1\right\} .
$$

Define

$$
\Psi=\Phi_{I} \cup \Phi_{t} .
$$

Lemma 7.4. (i) $\operatorname{rank} \mathcal{T}(\Psi)=\operatorname{rank} \mathcal{T}(\Phi)$.

(ii) For every $\varphi \in \mathcal{T}(\Phi)$ such that $\varphi \cap \Phi_{t} \in \mathcal{T}_{\text {aug }}\left(\Phi_{t}\right), \varphi \cap \Phi_{I} \in \mathcal{T}_{\text {aug }}\left(\Phi_{I}\right)$, and $\varphi \cap \Psi \in \mathcal{T}_{\text {aug }}(\Psi)$.

(iii) For every $\psi \in \mathcal{T}(\Psi)$ there is $\varphi \in \mathcal{T}(\Phi)$ such that $\varphi \cap \Psi=\psi$. Moreover, $\varphi$ is unique unless $\Phi$ is of type $C_{n}, n$ odd, and $\Psi$ has three irreducible factors of type $C_{r}, r$ odd.

(iv) Suppose that $\Phi$ is of type $A_{2 n}, n \geq 1$. Then $\Phi_{I}$ is of type $A_{2 s}$ for some $0 \leq s \leq n$. Otherwise, $\Psi$ contains no irreducible factors of type $A_{2 k}, k \geq 1$.

Proof. (i) and (ii) are elementary.

(iii) Let $\psi \in \mathcal{T}(\Psi)$. Then every irreducible factor of $\psi$ is of type $A_{1}$ or $B_{2}$, and $\operatorname{rank} \psi=\operatorname{rank} \mathcal{T}(\Psi)=\operatorname{rank} \mathcal{T}(\Phi)$, so $\psi \in \mathcal{T}_{\text {aug }}(\Phi)$. By Lemma 5.6 there is $\varphi \in \mathcal{T}(\Phi)$ such that $\psi \subset \varphi$. Then $\varphi \cap \Psi \in \mathcal{T}_{\text {aug }}(\Psi)$ and contains the two-structure $\psi$. Thus $\varphi \cap \Psi=\psi$.

Now suppose that $\varphi$ is not uniquely determined by $\psi$. By Lemma 5.6, $\Phi$ is of type $B_{n}, C_{n}$, or $F_{4}$. When $\Phi$ is of type $B_{n}, \Psi$ will be of the form $D_{2}^{k} \times D_{2 p} \times B_{r} \times B_{s}$ where $k, p, r, s \geq 0$ with $2 k+2 p+r+s=n$, and the $D_{2}, D_{2 p}$ factors consist of long roots in $\Phi$. Thus $\psi$ contains at most two irreducible factors of type $A_{1}$ generated by short roots coming from the $B_{r}$ and $B_{s}$ factors where $r$ or $s$ is odd. Thus by Lemma 5.6, $\varphi$ is uniquely determined by $\psi$.

When $\Phi$ is of type $F_{4}, \Psi$ will be of the form $F_{4}, B_{4}, B_{3} \times B_{1}, B_{2} \times B_{2}, B_{2} \times$ $A_{1}^{2}, B_{1}^{2} \times A_{1}^{2}$, or $C_{3} \times A_{1}$, where we use $A_{1}$ to denote an irreducible factor generated by one long root and $B_{1}$ to denote an irreducible factor generated by one short root. Thus $\psi$ will be of the form $B_{2}^{2}, B_{2} \times B_{1}^{2}, B_{2} \times A_{1}^{2}$, or $B_{1}^{2} \times A_{1}^{2}$. Again, by Lemma 5.6, $\mathcal{T}(\Phi, \psi)$ will contain exactly one element.

When $\Phi$ is of type $C_{n}, \Psi$ will be of the form $D_{2}^{k} \times C_{p} \times C_{r} \times C_{s}$ where $k, p, r, s \geq 0$ with $2 k+p+r+s=n$, and the $D_{2}$ factors are generated by short roots of $\Phi$. Thus $\psi$ has less than three irreducible factors of type $A_{1}$ generated by long roots, and hence $\varphi$ is unique by Lemma 5.6, unless $p, r, s$ are all odd. In this case $n$ must also be odd.

(iv) Since $\Phi_{t}$ is spanned by strongly orthogonal roots, it contains no irreducible factors of type $A_{2 k}, k \geq 1$. Further, $\Phi_{I}$ is the orthogonal complement in $\Phi$ of $S$. Now if $\Phi$ is spanned by orthogonal roots, so is $\Phi_{I}$, so it contains no $A_{2 k}$ factors. Suppose that $\Phi$ is not spanned by orthogonal roots. Then it is of type $A_{2 n}, A_{2 n+1}, D_{2 n+1}$, or $E_{6}$, and as in the proof of Theorem 5.3, the orthogonal complement of one root is of type $A_{2 n-2}, A_{2 n-1}, D_{2 n-1} \times A_{1}$, or $A_{5}$ respectively. Thus the orthogonal complement of an arbitrary orthogonal set $S$ is of type $A_{2 s}$ when $\Phi$ is of type $A_{2 n}$, and contains only simple factors of type $A_{2 k+1}, D_{2 k+1}$, or $E_{6}$ otherwise. 
Let $\Psi^{+}$be any choice of positive roots for $\Psi$. Then for any $\psi \in \mathcal{T}_{\text {aug }}(\Psi)$, we define

$$
\delta_{0}\left(\Psi^{+}, \psi, h\right)=\prod_{\alpha \in \Psi^{+} \backslash \psi^{+}} \delta(\alpha)
$$

where $\psi^{+}=\psi \cap \Psi^{+}$. For $\psi \in \mathcal{T}_{\text {aug }}(\Psi)$, let $\mathcal{T}(\Phi, \psi)=\{\varphi \in \mathcal{T}(\Phi): \varphi \cap \Psi=\psi\}$.

Lemma 7.5. Let $\Phi^{+}$be any choice of positive roots for $\Phi$ and let $\psi \in \mathcal{T}_{\text {aug }}(\Psi), \varphi \in$ $\mathcal{T}(\Phi, \psi)$. Then

$$
\delta\left(\Phi^{+}, \varphi, h\right)=e^{\rho\left(\Phi^{+}, \varphi\right)}(\gamma) \delta_{0}\left(\Psi \cap \Phi^{+}, \psi, h\right) \prod_{\alpha \in \Phi_{C P X}^{+} \backslash\left(\varphi \cap \Phi_{C P X}^{+}\right)} \delta(\alpha) .
$$

If in addition, $\Phi_{C P X}^{+}$is $\sigma$-stable, then

$$
\prod_{\alpha \in \Phi_{C P X}^{+} \backslash\left(\varphi \cap \Phi_{C P X}^{+}\right)} \delta(\alpha)=1 .
$$

Proof. Let $\varphi \in \mathcal{T}(\Phi, \psi)$. By Lemma 7.1,

$$
\delta\left(\Phi^{+}, \varphi, h\right)=e^{\rho\left(\Phi^{+}, \varphi\right)}(\gamma) \prod_{\alpha \in \Phi^{+} \backslash \varphi^{+}} \delta(\alpha) .
$$

Let $\alpha \in \Phi^{+} \backslash \Psi^{+}$. Then $\alpha \in\left(\Phi_{R} \backslash \Phi_{t}\right) \cup \Phi_{C P X}$. If $\alpha \in \Phi_{R} \backslash \Phi_{t}$, then $e^{-\alpha}(\gamma)=-1$ so that

$$
\Delta(\alpha)=e^{\frac{\alpha(H)}{2}}+e^{-\frac{\alpha(H)}{2}}
$$

is real and positive since $\alpha(H)$ is real. Thus $\delta(\alpha)=1$ in this case. Thus

$$
\prod_{\alpha \in \Phi^{+} \backslash \varphi^{+}} \delta(\alpha)=\delta_{0}\left(\Psi \cap \Phi^{+}, \Psi \cap \varphi, h\right) \prod_{\alpha \in \Phi_{C P X}^{+} \backslash \varphi \cap \Phi_{C P X}^{+}} \delta(\alpha) .
$$

Suppose that $\Phi_{C P X}^{+}$is $\sigma$-stable. Then $\Phi_{C P X}^{+}$can be divided into pairs $\alpha, \alpha^{\sigma, S}$. Since $e^{-\alpha}(\gamma)= \pm 1$ is real, we see that

$$
\Delta\left(\alpha^{\sigma, S}\right)=\overline{\Delta(\alpha)}
$$

Thus

$$
\delta\left(\alpha^{\sigma, S}\right) \delta(\alpha)=1
$$

Further, by Lemma 7.3, $\alpha \in \varphi$ if and only if $\alpha^{\sigma, S} \in \varphi$. Thus

$$
\prod_{\alpha \in \Phi_{C P X}^{+} \backslash \varphi \cap \Phi_{C P X}^{+}} \delta(\alpha)=1
$$

Lemma 7.6. Let $\Phi^{+}$be any choice of positive roots for $\Phi$ and let $\psi \in \mathcal{T}_{\text {aug }}(\Psi), \psi \notin$ $\mathcal{T}(\Psi)$. Then

$$
\sum_{\varphi \in \mathcal{T}(\Phi, \psi)} \epsilon\left(\varphi: \Phi^{+}\right) \delta\left(\Phi^{+}, \varphi, h\right)=0 .
$$


Proof. The statement is vacuous when $\Phi$ is of type $A_{2 n}$ since in this case $\Psi$ has one root length so that $\mathcal{T}_{\text {aug }}(\Psi)=\mathcal{T}(\Psi)$. Thus we can assume that $\Phi$ is not of type $A_{2 n}$. Further, since both sides of the equation are independent of $\Phi^{+}$by Lemma 7.2 , it is enough to prove the lemma for one choice of $\Phi^{+}$.

Since $\psi \notin \mathcal{T}(\Psi)$, by Lemma 5.6 there are $\alpha_{1}, \alpha_{2} \in \psi$ occurring in $A_{1}$ factors of $\psi$ such that $\alpha_{1}, \alpha_{2}$ are an orthogonal basis for root system of type $B_{2}$ in $\Psi$. Note that this implies that $\alpha_{1}, \alpha_{2}$ are either both in $\Phi_{t}$ or both in $\Phi_{I}$. Now $\alpha_{0}=c\left(\alpha_{1}-\alpha_{2}\right) \in \Psi$ where $c \in\{1,1 / 2\}$. Let $s$ denote the reflection corresponding to $\alpha_{0}$. Then $s$ interchanges $\alpha_{1}$ and $\alpha_{2}$, and fixes every root of $\Psi$ orthogonal to both $\alpha_{1}$ and $\alpha_{2}$. In particular, $s \psi=\psi$.

Let $\mathcal{B}=\left\{\beta_{1}, \ldots, \beta_{n}\right\}$ be an ordered orthogonal basis of $\psi$ such that $\beta_{1}, \ldots, \beta_{r}$ is a basis for $\psi \cap \Phi_{t}$ and $\beta_{r+1}, \ldots, \beta_{n}$ is a basis for $\psi \cap \Phi_{I}$. Further, if $\alpha_{1}, \alpha_{2} \in \Phi_{t}$, then we take $\beta_{i}=\alpha_{i}, i=1,2$, while if $\alpha_{1}, \alpha_{2} \in \Phi_{I}$, we take $\beta_{n-1}=\alpha_{1}, \beta_{n}=\alpha_{2}$. Let $\Phi^{+}=\Phi^{+}(\mathcal{B})$. Then $\Phi_{C P X}^{+}$is $\sigma$-stable. Let $\varphi \in \mathcal{T}(\Phi, \psi)$, and suppose that there is $\alpha \in \varphi^{+}$such that $s \alpha \in \Phi^{-}$. Since $s$ interchanges $\alpha_{1}$ and $\alpha_{2}$ and fixes every other $\beta_{i} \in \mathcal{B}$, we must have $\left\langle\alpha, \alpha_{1}\right\rangle>0,\left\langle\alpha, \alpha_{2}\right\rangle<0$. In particular, we must have $\alpha_{1}, \alpha_{2}$ in the same simple factor of $\varphi$. But then $\alpha_{0} \in \varphi \cap \Psi=\psi$. This contradicts the assumption that $\alpha_{1}, \alpha_{2}$ occur in $A_{1}$ factors of $\psi$. Thus $s \varphi^{+} \subset \Phi^{+}$ for all $\varphi \in \mathcal{T}(\Phi, \psi)$.

Let $\varphi \in \mathcal{T}(\Phi, \psi)$. Then $s \varphi \cap \Psi=s(\varphi \cap \Psi)=s \psi=\psi$, so that $s \varphi \in \mathcal{T}(\Phi, \psi)$ also. Further, since $s^{2}=1, s(s \varphi)=\varphi$. Thus we can divide $\mathcal{T}(\Phi, \psi)$ into pairs, $\varphi, s \varphi$. But by Lemma 5.2 ,

$$
\epsilon\left(s \varphi: \Phi^{+}\right)=\operatorname{det} s \epsilon\left(\varphi: \Phi^{+}\right)=-\epsilon\left(\varphi: \Phi^{+}\right) .
$$

Further

$$
\rho\left(\Phi^{+}, s \varphi\right)=\rho\left(\Phi^{+}, \varphi\right)+\rho\left(\varphi^{+}\right)-s \rho\left(\varphi^{+}\right) .
$$

Since $\rho\left(\varphi^{+}\right)$is a weight for $\Phi$ and hence for $\Psi, \rho\left(\varphi^{+}\right)-s \rho\left(\varphi^{+}\right)$is in the root lattice of $\Psi$, so that

$$
e^{\rho\left(\Phi^{+}, s \varphi\right)}(\gamma)=e^{\rho\left(\Phi^{+}, \varphi\right)}(\gamma) e^{\rho\left(\varphi^{+}\right)-s \rho\left(\varphi^{+}\right)}(\gamma)=e^{\rho\left(\Phi^{+}, \varphi^{+}\right)}(\gamma) .
$$

Then, by Lemma 7.5 , since $\Phi_{C P X}^{+}$is $\sigma$-stable,

$$
\begin{aligned}
& \delta\left(\Phi^{+}, s \varphi, h\right)=e^{\rho\left(\Phi^{+}, s \varphi\right)}(\gamma) \delta_{0}\left(\Psi \cap \Phi^{+}, \psi, h\right) \\
& =e^{\rho\left(\Phi^{+}, \varphi\right)}(\gamma) \delta_{0}\left(\Psi \cap \Phi^{+}, \psi, h\right)=\delta\left(\Phi^{+}, \varphi, h\right) .
\end{aligned}
$$

Thus

$$
\epsilon\left(s \varphi: \Phi^{+}\right) \delta\left(\Phi^{+}, s \varphi, h\right)+\epsilon\left(\varphi: \Phi^{+}\right) \delta\left(\Phi^{+}, \varphi, h\right)=0
$$

for all $\varphi \in \mathcal{T}(\Phi, \psi)$.

Fix $\psi \in \mathcal{T}(\Psi)$, and $\varphi \in \mathcal{T}(\Phi, \psi)$. Let $\mathcal{B}$ be a basis for $\psi$ which contains two long orthogonal roots from every irreducible factor of $\psi$ of type $B_{2}$.

Lemma 7.7. There is an ordering $\mathcal{B}=\left\{\alpha_{1}, \ldots, \alpha_{n}\right\}$ with the following properties.

(i) For every irreducible factor $\varphi_{j}$ of $\varphi$, the roots in $\mathcal{B} \cap \varphi_{j}$ are adjacent in the ordering.

(ii) Let $\Phi$ be of type $C_{n}, n$ odd, and let $\alpha_{s}$ be the root in $\mathcal{B}$ which generates the unique irreducible factor of $\varphi$ of type $C_{1}$. Then if $\alpha_{s} \in \Phi_{t}$, it is the first root in $\mathcal{B}$, while if $\alpha_{s} \in \Phi_{I}$, then it is the last root in $\mathcal{B}$. 
(iii) Let $\Phi^{+}=\Phi^{+}(\mathcal{B})$. Then

$$
\prod_{\alpha \in \Phi_{C P X}^{+} \backslash \varphi \cap \Phi_{C P X}^{+}} \delta(\alpha)=1 .
$$

Proof. Let $\Psi_{i}$ be an irreducible factor of $\Psi$. We will call $\Psi_{i}$ imaginary, respectively real, if it is contained in $\Phi_{I}$, respectively $\Phi_{t}$. Since $\Psi=\Phi_{I} \cup \Phi_{t}$ every $\Psi_{i}$ is either real or imaginary. Similarly, we call every element of $\Phi_{t}$ a real root of $\Psi$ and every element of $\Phi_{I}$ an imaginary root of $\Psi$.

Suppose first that $\varphi$ contains no irreducible factors of type $B_{2}$. Then conditions (i) and (ii) are vacuous, so that it suffices to produce an ordering of $\mathcal{B}$ which satisfies (iii). For this we just need to order $\mathcal{B}$ so that the real roots precede the imaginary roots, so that $\Phi_{C P X}^{+}$is $\sigma$-stable.

Now suppose that $\varphi$ contains an irreducible factor of type $B_{2}$, so that $\Phi$ is of type $B_{n}, C_{n}$, or $F_{4}$. Let $\Psi_{i}, i=1,2$, be distinct irreducible factors of $\Psi$. We will say they are linked if there is an irreducible factor $\varphi_{j}$ of $\varphi$ of type $B_{2}$ which has nonempty intersection with both. In this case $\varphi_{j} \cap \Psi_{i}$ is an irreducible factor of $\psi_{i}=\psi \cap \Psi_{i}$ of type $A_{1}$, and the roots in $\varphi_{j} \cap \Psi_{1}$ and $\varphi_{j} \cap \Psi_{2}$ have the same length in $\Phi$.

Suppose that $\Phi$ is of type $B_{n}$. Then $\Phi_{I}$ is of type $B_{r} \times A_{1}^{k}$ and $\Phi_{t}$ is of type $A_{1}^{k} \times D_{2 p} \times B_{q}$ where $r+2 k+2 p+q=n$. Let $\Psi_{2 i-1}, 1 \leq i \leq k$, denote the irreducible factors of $\Phi_{t}$ of type $A_{1}$. Then for each $1 \leq i \leq k$ there is a unique irreducible factor $\Psi_{2 i}$ of $\Phi_{I}$ of type $A_{1}$ which is linked with $\Psi_{2 i-1}$. The irreducible factor $\Psi_{2 k+1}$ of $\Phi_{t}$ of type $D_{2 p}$ cannot be linked to another factor. Finally, the factors $\Psi_{2 k+2}$ of $\Phi_{t}$ of type $B_{q}$ and $\Psi_{2 k+3}$ of $\Phi_{I}$ of type $B_{r}$ are linked just in case $q$ and $r$ are both odd. Now $\Psi_{1}, \ldots, \Psi_{2 k+3}$ is an ordering of the irreducible factors of $\Psi$ with the property that linked factors are adjacent.

Now we order $\mathcal{B}$ as follows. If $\Psi_{i}$ is not linked to any other irreducible factors, then we order $\mathcal{B}_{i}=\Psi_{i} \cap \mathcal{B}$ so that if two roots are in the same irreducible factor of $\varphi$ they are adjacent in the ordering. If $\Psi_{i}, \Psi_{i+1}$ are linked, we order $\mathcal{B}_{i}$ so that if two roots are in the same irreducible factor of $\varphi$ they are adjacent in the ordering, and the linked root is last. We order $\mathcal{B}_{i+1}$ so that if two roots are in the same irreducible factor of $\varphi$ they are adjacent in the ordering, and the linked root is first. Now order $\mathcal{B}$ by taking $\mathcal{B}=\mathcal{B}_{1}, \mathcal{B}_{2}, \ldots, \mathcal{B}_{2 k+3}$. This produces an ordering for $\mathcal{B}$ which satisfies (i).

Let $\alpha \in \Phi_{C P X}$ and let $\alpha_{i}$ be the first element of $\mathcal{B}$ such that $\left\langle\alpha, \alpha_{i}\right\rangle \neq 0$. Suppose that $\alpha_{i} \in \Phi_{I}$. If $\alpha_{i} \in \Psi_{2 k+3}$, then $\alpha$ is orthogonal to every real root of $\mathcal{B}$, so that $\alpha \in \Phi_{I}$. Suppose that $\alpha_{i} \in \Psi_{2 j}$ for some $1 \leq j \leq k$. Write $\mathcal{B}_{2 j-1}=\{\beta\}$. Now

$$
\alpha \in \Phi_{\beta}=\{\gamma \in \Phi:\langle\gamma, \beta\rangle=0\} .
$$

Since $\beta$ is a long root and $\Phi$ is of type $B_{n}, \Phi_{\beta}$ is of type $A_{1} \times B_{n-2}$. Here the factor of type $A_{1}$ is $\Psi_{2 j}$ and the factor of type $B_{n-2}$ is the set of all $\gamma \in \Phi$ which are orthogonal to both $\beta$ and $\alpha_{i}$. Since $\left\langle\alpha, \alpha_{i}\right\rangle \neq 0$, we must have $\alpha= \pm \alpha_{i} \in \Phi_{I}$. These contradictions show that $\alpha_{i} \in \Phi_{t}$. Thus $\Phi_{C P X}^{+}$is $\sigma$-stable so that (iii) is satisfied.

Suppose that $\Phi$ is of type $C_{n}$. Then $\Phi_{I}$ is of type $C_{r} \times A_{1}^{k}$ and $\Phi_{t}$ is of type $A_{1}^{k} \times C_{p} \times C_{q}$ where $r+2 k+p+q=n$. Define $\Psi_{i}, 1 \leq i \leq 2 k$, as in the $B_{n}$ case. When $n$ is odd we let $\alpha_{s}$ denote the root of $\mathcal{B}$ which generates the unique irreducible factor of $\varphi_{0}$ of type $A_{1}$. 
Suppose that $n$ is even, or that $n$ is odd and $\alpha_{s} \in \Phi_{I}$. Let $\Psi_{2 k+3}$ be the irreducible factor of $\Phi_{I}$ of type $C_{r}$. The factors of $\Phi_{t}$ of type $C_{p}$ and $C_{q}$ should be labeled $\Psi_{2 k+1}$ and $\Psi_{2 k+2}$. If $\Psi_{2 k+3}$ is linked to one of these two factors, then it should be labeled $\Psi_{2 k+2}$, while the remaining factor is $\Psi_{2 k+1}$. Now $\Psi_{1}, \ldots, \Psi_{2 k+3}$ is an ordering of the irreducible factors of $\Psi$ with the property that linked factors are adjacent. We now order $\mathcal{B}$ as in the $B_{n}$ case. In addition, if $n$ is odd, then $\alpha_{s} \in \Psi_{2 k+3}$ and this factor is not linked to any other. Thus we can order the roots in $\mathcal{B} \cap \Psi_{2 k+3}$ so that $\alpha_{s}$ is last. Using the argument in the $B_{n}$ case, with the roles of long and short roots reversed we see that this ordering satisfies (iii).

Suppose that $n$ is odd, and $\alpha_{s} \in \Phi_{t}$. Then it is in one of the the factors of $\Phi_{t}$ of type $C_{p}, C_{q}$. This factor should be labelled $\Psi_{0}$. It is not linked to any other factor. The other factor of this type should be labelled $\Psi_{2 k+1}$ and the irreducible factor of $\Phi_{I}$ of type $C_{r}$ should be labelled $\Psi_{2 k+2}$. Now $\Psi_{0}, \ldots, \Psi_{2 k+2}$ is an ordering of the irreducible factors of $\Psi$ with the property that linked factors are adjacent. Order $\mathcal{B}$ as usual. Since $\Psi_{0}$ is not linked, we can order the roots in $\mathcal{B} \cap \Psi_{0}$ so that $\alpha_{s}$ is first, and as above this ordering satisfies (iii).

Finally, suppose that $\Phi$ is of type $F_{4}$. If $\Phi_{I}$ consists of only one irreducible factor, then we can easily order the irreducible factors of $\Psi$ so that any linked irreducible factors are adjacent, and the imaginary factor is last. As above this yields an ordering of $\mathcal{B}$ satisfying (i) and (iii).

Now suppose that $\Phi$ is of type $F_{4}$ and $\Phi_{I}$ is reducible. This occurs only when $S$ consists of two roots, one short and one long. In this case $\Psi=\left\{ \pm \alpha_{i}, 1 \leq i \leq 4\right\}$ is of type $B_{1}^{2} \times A_{1}^{2}$ and we can write $\Phi_{t}=\Psi_{1} \cup \Psi_{3}$ and $\Phi_{I}=\Psi_{2} \cup \Psi_{4}$ where $\Psi_{i}=\left\{ \pm \alpha_{i}\right\}, 1 \leq i \leq 4$. We will order the roots so that $\alpha_{1}$ and $\alpha_{2}$ have the same length and $\alpha_{3}$ and $\alpha_{4}$ have the same length. Then $\Psi_{2 i-1}$ and $\Psi_{2 i}$ must be linked, $1 \leq i \leq 2$, so this ordering satisfies (i).

Let $\beta \in \Phi_{C P X}^{+}$, and assume that $-\beta^{\sigma, S} \in \Phi^{+}$. Then we must have $\left\langle\beta, \alpha_{1}\right\rangle=0$ and $\left\langle\beta, \alpha_{2}\right\rangle \neq 0$. There are four roots of this type given by

$$
\begin{gathered}
\beta_{1}=c_{2} \alpha_{2}+c_{3}\left(\alpha_{3}+\alpha_{4}\right), \quad \beta_{2}=c_{2} \alpha_{2}+c_{3}\left(\alpha_{3}-\alpha_{4}\right), \\
\beta_{3}=c_{2} \alpha_{2}+c_{3}\left(-\alpha_{3}+\alpha_{4}\right), \quad \beta_{4}=c_{2} \alpha_{2}+c_{3}\left(-\alpha_{3}-\alpha_{4}\right)
\end{gathered}
$$

where $c_{2}, c_{3} \in\{1 / 2,1\}$. Further, $\beta_{3}=-\beta_{1}^{\sigma, S}$ and $\beta_{4}=-\beta_{2}^{\sigma, S}$. Now, since for $i=1,2$,

$$
\delta\left(\beta_{i}\right) \delta\left(-\beta_{i}^{\sigma, S}\right)=-e^{\beta_{i}}(\gamma), \quad \prod_{1=1}^{4} \delta\left(\beta_{i}\right)=e^{\beta_{1}+\beta_{2}}(\gamma) .
$$

But $\beta_{1}+\beta_{2}=2 c_{2} \alpha_{2}+2 c_{3} \alpha_{3}$ and $\gamma=\exp \left(H_{0}\right)$ where $H_{0}=\pi i\left(n_{1} H_{\alpha_{1}}^{*}+n_{3} H_{\alpha_{3}}^{*}\right)$ with $n_{i} \in \mathbf{Z}, i=1,3$. Thus

$$
\left(\beta_{1}+\beta_{2}\right)\left(H_{0}\right)=2 \pi i 2 c_{3} n_{3} \in 2 \pi i \mathbf{Z}
$$

so that

$$
\prod_{1=1}^{4} \delta\left(\beta_{i}\right)=1
$$

Now $\varphi \cap \Psi=\psi=\Psi$ since all irreducible factors of $\Psi$ are of type $A_{1}$. Since the orthogonal roots in a factor of type $B_{2}$ must have the same length, $\varphi=\varphi_{1} \cup \varphi_{2}$ 
where $\varphi_{i}$ is the unique factor of type $B_{2}$ containing $\alpha_{2 i-1}$ and $\alpha_{2 i}, i=1,2$. Thus $\beta_{i} \notin \varphi, 1 \leq i \leq 4$. Since for all other $\alpha \in \Phi_{C P X}^{+}, \alpha^{\sigma, S} \in \Phi^{+}$we have

$$
\prod_{\alpha \in \Phi_{C P X}^{+} \backslash\left(\varphi \cap \Phi_{C P X}^{+}\right)} \delta(\alpha)=1
$$

For any $\psi \in \mathcal{T}(\Psi)$ and choice $\Psi^{+}$of positive roots for $\Psi$, we have defined

$$
\delta_{0}\left(\Psi^{+}, \psi, h\right)=\prod_{\alpha \in \Psi^{+} \backslash\left(\psi \cap \Psi^{+}\right)} \delta(\alpha) .
$$

If $\Phi$ is not of type $A_{2 n}$, then by Theorem 5.5 and Lemma 7.4(iv), $\rho\left(\Psi^{+}, \psi\right)$ is in the root lattice of $\Psi$ so that

$$
e^{\rho\left(\Psi^{+}, \psi\right)}(\gamma)=1
$$

When $\Phi$ is of type $A_{2 n}, e^{\rho\left(\Psi^{+}, \psi\right)}(\gamma)=1$ by definition. Thus we have

$$
\delta_{0}\left(\Psi^{+}, \psi, h\right)=\delta\left(\Psi^{+}, \psi, h\right)=e^{\rho\left(\Psi^{+}, \psi\right)}(\gamma) \prod_{\alpha \in \Psi^{+} \backslash\left(\psi \cap \Psi^{+}\right)} \delta(\alpha) .
$$

By Lemma 7.2 applied to $\Psi$, we see that $\epsilon\left(\psi: \Psi^{+}\right) \delta\left(\Psi^{+}, \psi, h\right)$ is independent of the choice of $\Psi^{+}$.

Lemma 7.8. Let $\Phi^{+}$and $\Psi^{+}$be any choices of positive roots and let $\psi \in \mathcal{T}(\Psi)$. Then

$$
\sum_{\varphi \in \mathcal{T}(\Phi, \psi)} \epsilon\left(\varphi: \Phi^{+}\right) \delta\left(\Phi^{+}, \varphi, h\right)=\epsilon\left(\psi: \Psi^{+}\right) \delta\left(\Psi^{+}, \psi, h\right) .
$$

Proof. Fix $\varphi \in \mathcal{T}(\Phi, \psi)$, and let $\mathcal{B}(\varphi)=\left\{\alpha_{1}, \ldots, \alpha_{n}\right\}$ be an ordered basis for $\psi$ satisfying the conditions in Lemma 7.7 with respect to $\varphi$. Let

$$
\Phi^{+}(\varphi)=\Phi^{+}(\mathcal{B}(\varphi)), \quad \Psi^{+}(\varphi)=\Psi^{+}(\mathcal{B}(\varphi)) .
$$

Then $\epsilon\left(\psi: \Psi^{+}(\varphi)\right)=1$ by definition. Further,

$$
\delta\left(\Phi^{+}(\varphi), \varphi, h\right)=e^{\rho\left(\Phi^{+}(\varphi), \varphi\right)}(\gamma) \delta\left(\Psi^{+}(\varphi), \psi, h\right)
$$

by Lemma 7.5 .

Modify $\mathcal{B}=\mathcal{B}(\varphi)$ as follows. If $\varphi_{i}$ is an irreducible factor of $\varphi$ of type $A_{1}$, then $\mathcal{B} \cap \varphi_{i}=\left\{\alpha_{p}\right\}$ for some $1 \leq p \leq n$, and we set $\beta_{p}=\alpha_{p}$. If $\varphi_{i}$ is an irreducible factor of $\varphi$ of type $B_{2}, \mathcal{B} \cap \varphi_{i}=\left\{\alpha_{p}, \alpha_{p+1}\right\}$ for some $1 \leq p \leq n-1$. The two roots have consecutive indices by definition, and since they are orthogonal in a root system of type $B_{2}$, either both are short or both are long. If both are long, then we define $\beta_{p}=\alpha_{p}$ and $\beta_{p+1}=\alpha_{p+1}$. However if both are short, we replace them by $\beta_{p}=\alpha_{p}+\alpha_{p+1}$ and $\beta_{p+1}=\alpha_{p}-\alpha_{p+1}$. This produces an ordered basis $\mathcal{B}^{\prime}=\left\{\beta_{1}, \ldots, \beta_{n}\right\}$, for $\varphi$ which contains two orthogonal long roots from every irreducible factor of $\varphi$ of type $B_{2}$, and they are adjacent in the ordering. By definition, $\epsilon\left(\varphi: \Phi^{+}\left(\mathcal{B}^{\prime}\right)\right)=1$. But it is easy to check that $\Phi^{+}(\mathcal{B})=\Phi^{+}\left(\mathcal{B}^{\prime}\right)$. Thus $\epsilon\left(\varphi: \Phi^{+}(\varphi)\right)=1$. 
Now by Lemma 7.2 , for any $\Phi^{+}$and $\Psi^{+}$we have

$$
\begin{aligned}
& \sum_{\varphi \in \mathcal{T}(\Phi, \psi)} \epsilon\left(\varphi: \Phi^{+}\right) \delta\left(\Phi^{+}, \varphi, h\right)=\sum_{\varphi} \epsilon\left(\varphi: \Phi^{+}(\varphi)\right) \delta\left(\Phi^{+}(\varphi), \varphi, h\right) \\
& =\sum_{\varphi} e^{\rho\left(\Phi^{+}(\varphi), \varphi\right)}(\gamma) \delta\left(\Psi^{+}(\varphi), \psi, h\right) \epsilon\left(\psi: \Psi^{+}(\varphi)\right) \\
& =\epsilon\left(\psi: \Psi^{+}\right) \delta\left(\Psi^{+}, \psi, h\right) \sum_{\varphi} e^{\rho\left(\Phi^{+}(\varphi), \varphi\right)}(\gamma) .
\end{aligned}
$$

Thus it suffices to prove that

$$
\sum_{\varphi \in \mathcal{T}(\Phi, \psi)} e^{\rho\left(\Phi^{+}(\varphi), \varphi\right)}(\gamma)=1
$$

Suppose that $\Phi$ is not of type $C_{2 m+1}, m \geq 1$. Then by Lemma 7.4, $\mathcal{T}(\Phi, \psi)=\{\varphi\}$ has only one element. Further, by Lemma 5.4 , if $\Phi$ is not of type $A_{2 n}, \rho\left(\Phi^{+}(\varphi), \varphi\right)$ is in the root lattice of $\Psi$ since each $\beta_{i} \in \mathcal{B}^{\prime}$ is. Thus $e^{\rho\left(\Phi^{+}(\varphi), \varphi\right)}(\gamma)=1$. If $\Phi$ is of type $A_{2 n}$, then $e^{\rho\left(\Phi^{+}(\varphi), \varphi\right)}(\gamma)=1$ by definition.

Suppose that $\Phi$ is of type $C_{2 m+1}$, and fix $\varphi \in \mathcal{T}(\Phi, \psi)$. Let $\alpha_{0}$ denote the element of $\mathcal{B}$ which generates the irreducible factor of $\varphi$ of type $C_{1}$. If $\alpha_{0} \in \Phi_{t}$, then it is first in $\mathcal{B}^{\prime}$. Thus again by Lemma $5.4 \rho\left(\Phi^{+}, \varphi\right)$ is in the root lattice of $\Psi$ so that as above

$$
e^{\rho\left(\Phi^{+}(\varphi), \varphi\right)}(\gamma)=1
$$

Suppose that $\alpha_{0} \in \Phi_{I}$. Then it is last in $\mathcal{B}^{\prime}$ and by Lemma 5.4,

$$
\rho\left(\Phi^{+}(\varphi), \varphi\right)=\frac{1}{2} \sum_{i=1}^{2 m} \beta_{i}+\lambda
$$

where $\lambda$ is in the root lattice of $\Psi$. Thus

$$
e^{\rho\left(\Phi^{+}(\varphi), \varphi\right)}(\gamma)=e^{(1 / 2) \sum_{i=1}^{2 m} \beta_{i}}(\gamma)
$$

Now $\Phi_{I}$ is of type $A_{1}^{k} \times C_{r}$ and $\Phi_{t}$ is of type $A_{1}^{k} \times C_{p} \times C_{q}$, and by the choice of $\mathcal{B}$ in Lemma 7.7, $\delta_{i}=\frac{1}{2}\left(\beta_{2 i-1}+\beta_{2 i}\right) \in \Psi$ for all $1 \leq i \leq m$, except in the case that $p$ and $q$ are both odd, in which case exactly one $\delta_{i} \in \Phi_{R} \backslash \Phi_{t}$. Thus

$$
e^{\rho\left(\Phi^{+}(\varphi), \varphi\right)}(\gamma)= \begin{cases}-1, & \text { if } p \text { and } q \text { are both odd } \\ 1, & \text { otherwise. }\end{cases}
$$

Now if exactly one of $p, q, r$ is odd, then $\mathcal{T}(\Phi, \psi)=\{\varphi\}$ has only one element and $e^{\rho\left(\Phi^{+}(\varphi), \varphi\right)}(\gamma)=1$. If all three of $p, q, r$ are odd, then $\mathcal{T}(\Phi, \psi)=\left\{\varphi_{i}, 1 \leq k \leq 3\right\}$. For two of these elements, say $\varphi_{1}$ and $\varphi_{2}$, the unique irreducible factor of type $C_{1}$ is contained in $\Phi_{t}$. For the other, $\varphi_{3}$, the unique irreducible factor of type $C_{1}$ is contained in $\Phi_{I}$. Thus by the above,

$$
\sum_{i=1}^{3} e^{\rho\left(\Phi^{+}\left(\varphi_{i}\right), \varphi_{i}\right)}(\gamma)=1+1-1=1
$$

Lemma 7.9. There is a choice $\Psi^{+}(h)$ of positive roots for $\Psi$ such that $\Psi^{+}(h) \cap \Phi_{t}=$ $\Phi_{R}^{+}(h)$ and

$$
\delta\left(\Psi^{+}(h), \psi, h\right)=(-1)^{r\left(\Phi_{I}\right)}
$$

for every $\psi \in \mathcal{T}(\Psi)$. Here $r\left(\Phi_{I}\right)$ is defined as in Theorem 5.5. 
Proof. Recall that for any choice of $\Psi^{+}, \psi \in \mathcal{T}(\Psi), \psi^{+}=\psi \cap \Psi^{+}$,

$$
\delta\left(\Psi^{+}, \psi, h\right)=\delta_{0}\left(\Psi^{+}, \psi, h\right)=\prod_{\alpha \in \Psi^{+} \backslash \psi^{+}} \delta(\alpha) .
$$

We have $h=\gamma \exp \left(c_{S} H\right)$ where $H=H_{1}+H_{2}, H_{1} \in \underline{t}_{S}, H_{2}=c_{S}^{-1} \log a$. Now for all $\alpha \in \Psi, e^{\alpha}(\gamma)=1$, so that

$$
\Delta(\alpha)=\exp (\alpha(H) / 2)-\exp (-\alpha(H) / 2) .
$$

Suppose that $\alpha \in \Phi_{R}^{+}(h)$. Then $\alpha(H)=\alpha\left(H_{2}\right)=\alpha\left(c_{S}^{-1} \log a\right)$ is real and positive. Thus $\Delta(\alpha)=2 \sinh \left(\alpha\left(H_{2}\right) / 2\right)$ is real and positive, so that $\delta(\alpha)=1$. Let $\Phi_{I}^{+}$be any choice of positive roots for $\Phi_{I}$, and write $\psi_{I}^{+}=\psi \cap \Phi_{I}^{+}$. Then if $\Psi^{+}=\Phi_{R}^{+}(h) \cup \Phi_{I}^{+}$, we have

$$
\delta\left(\Psi^{+}, \psi, h\right)=\prod_{\alpha \in \Phi_{I}^{+} \backslash \psi_{I}^{+}} \delta(\alpha) .
$$

Define

$$
L_{S}=\left\{H \in \underline{t}_{S}: \alpha(H) \in 2 \pi i \mathbf{Z} \forall \alpha \in \Phi_{I}\right\} .
$$

Then we can write $H_{1}=H_{1}^{\prime}+H_{0}^{\prime}$ where $H_{0}^{\prime} \in L_{S}$ and $H_{1}^{\prime} \in \underline{t}_{S}$ satisfies $\left|\alpha\left(H_{1}^{\prime}\right)\right| \leq 2 \pi$ for all $\alpha \in \Phi_{I}$. When $\Phi$ is of type $A_{2 n}$, we have $H_{1}+c_{S} H_{2} \in \omega$, so that for $\alpha \in \Phi_{I}$, $\left|\alpha\left(H_{1}\right)\right|=\left|\operatorname{Im} \alpha\left(H_{1}+c_{S} H_{2}\right)\right|<\pi$. Thus in this case we can take $H_{1}=H_{1}^{\prime}, H_{0}^{\prime}=0$. For $\alpha \in \Phi_{I}$, write

$$
\Delta^{\prime}(\alpha)=\exp \left(\alpha\left(H_{1}^{\prime}\right) / 2\right)-\exp \left(-\alpha\left(H_{1}^{\prime}\right) / 2\right), \quad \delta^{\prime}(\alpha)=\Delta^{\prime}(\alpha)\left|\Delta^{\prime}(\alpha)\right|^{-1} .
$$

For every $\alpha \in \Phi_{I}(S), \alpha\left(H_{0}^{\prime}\right) \in 2 \pi i \mathbf{Z}$, so that

$$
\exp \left(\alpha\left(H_{0}^{\prime}\right) / 2\right)=\exp \left(-\alpha\left(H_{0}^{\prime}\right) / 2\right) .
$$

Thus

$$
\begin{gathered}
\Delta(\alpha)=\exp \left(\alpha\left(H_{1}\right) / 2\right)-\exp \left(-\alpha\left(H_{1}\right) / 2\right)=\exp \left(\alpha\left(H_{0}^{\prime}\right) / 2\right) \Delta^{\prime}(\alpha) ; \\
\prod_{\alpha \in \Phi_{I}^{+} \backslash \psi_{I}^{+}} \Delta(\alpha)=\exp \left(\left(\rho\left(\Phi_{I}^{+}\right)-\rho\left(\psi_{I}^{+}\right)\right)\left(H_{0}^{\prime}\right)\right) \prod_{\alpha \in \Phi_{I}^{+} \backslash \psi_{I}^{+}} \Delta^{\prime}(\alpha) .
\end{gathered}
$$

When $\Phi$ is of type $A_{2 n}, H_{0}^{\prime}=0$. Otherwise, for every $\psi \in \mathcal{T}(\Psi), \psi_{I} \in \mathcal{T}\left(\Phi_{I}\right)$ so that by Theorem 5.5 and Lemma 7.4(iv), $\rho\left(\Phi_{I}^{+}\right)-\rho\left(\psi_{I}^{+}\right)$is in the root lattice of $\Phi_{I}$. Thus in any case we have

$$
\exp \left(\left(\rho\left(\Phi_{I}^{+}\right)-\rho\left(\psi_{I}^{+}\right)\right)\left(H_{0}^{\prime}\right)\right)=1 ; \quad \delta\left(\Psi^{+}, \psi, h\right)=\prod_{\alpha \in \Phi_{I}^{+} \backslash \psi_{I}^{+}} \delta^{\prime}(\alpha) .
$$

Let $\alpha \in \Phi_{I}$. Then $t_{\alpha}=\alpha\left(-i H_{1}^{\prime}\right) / 2$ is real, and $\Delta^{\prime}(\alpha)=2 i \sin t_{\alpha}$. By our choice of $H_{1}^{\prime}$, we have $-\pi<t_{\alpha}<\pi$. Further, since $\Delta(\alpha) \neq 0$, we have $t_{\alpha} \neq 0$, so we can define a choice $\Phi_{I}^{+}(h)$ of positive roots for $\Phi_{I}$ by $\alpha \in \Phi_{I}^{+}(h)$ if and only if $t_{\alpha}>0$. Now for $\alpha \in \Phi_{I}^{+}(h), 0<t_{\alpha}<\pi$ so that $\sin t_{\alpha}>0$ and $\delta^{\prime}(\alpha)=i$.

Let $\Psi^{+}(h)=\Phi_{I}^{+}(h) \cup \Phi_{R}^{+}(h)$. For $\psi \in \mathcal{T}(\Psi), 2 r\left(\Phi_{I}\right)=\left[\Phi_{I}^{+}(h) \backslash\left(\psi \cap \Phi_{I}^{+}(h)\right)\right]$ by definition. Thus by the above,

$$
\delta\left(\Psi^{+}(h), \psi, h\right)=i^{2 r\left(\Phi_{I}\right)}=(-1)^{r\left(\Phi_{I}\right)} .
$$


Proof of Lemma 6.8. Let $\psi_{t} \in \mathcal{T}_{\text {aug }}\left(\Phi_{t}\right)$. Then we must show that for any choice of $\Phi^{+}$,

$$
\sum_{\varphi \in \mathcal{T}\left(\Phi, \psi_{t}\right)} \epsilon\left(\varphi: \Phi^{+}\right) \delta\left(\Phi^{+}, \varphi, h\right)= \begin{cases}\epsilon\left(\psi_{t}: \Phi_{R}^{+}(h)\right), & \text { if } \psi_{t} \in \mathcal{T}\left(\Phi_{t}\right) \\ 0, & \text { otherwise. }\end{cases}
$$

Fix $\psi_{t} \in \mathcal{T}_{\text {aug }}\left(\Phi_{t}\right)$. For each $\varphi \in \mathcal{T}\left(\Phi, \psi_{t}\right), \varphi \cap \Psi=\psi_{I} \cup \psi_{t}$ where $\psi_{I} \in \mathcal{T}_{\text {aug }}\left(\Phi_{I}\right)$ by Lemma 7.4. We can write

$$
\sum_{\varphi \in \mathcal{T}\left(\Phi, \psi_{t}\right)} \epsilon\left(\varphi: \Phi^{+}\right) \delta\left(\Phi^{+}, \varphi, h\right)=\sum_{\psi_{I} \in \mathcal{T}_{\text {aug }}\left(\Phi_{I}\right)} \sum_{\varphi \in \mathcal{T}\left(\Phi, \psi_{I} \cup \psi_{t}\right)} \epsilon\left(\varphi: \Phi^{+}\right) \delta\left(\Phi^{+}, \varphi, h\right) .
$$

But by Lemma 7.6 ,

$$
\sum_{\varphi \in \mathcal{T}\left(\Phi, \psi_{I} \cup \psi_{t}\right)} \epsilon\left(\varphi: \Phi^{+}\right) \delta\left(\Phi^{+}, \varphi, h\right)=0
$$

unless $\psi_{I} \cup \psi_{t} \in \mathcal{T}(\Psi)$. In particular, if $\psi_{t} \notin \mathcal{T}\left(\Phi_{t}\right)$, we have

$$
\sum_{\varphi \in \mathcal{T}\left(\Phi, \psi_{t}\right)} \epsilon\left(\varphi: \Phi^{+}\right) \delta\left(\Phi^{+}, \varphi, h\right)=0
$$

and if $\psi_{t} \in \mathcal{T}\left(\Phi_{t}\right)$, we only get a nonzero contribution from $\psi_{I} \in \mathcal{T}\left(\Phi_{I}\right)$.

Suppose that $\psi_{t} \in \mathcal{T}\left(\Phi_{t}\right)$. Then by the above,

$$
\sum_{\varphi \in \mathcal{T}\left(\Phi, \psi_{t}\right)} \epsilon\left(\varphi: \Phi^{+}\right) \delta\left(\Phi^{+}, \varphi, h\right)=\sum_{\psi_{I} \in \mathcal{T}\left(\Phi_{I}\right)} \sum_{\varphi \in \mathcal{T}\left(\Phi, \psi_{I} \cup \psi_{t}\right)} \epsilon\left(\varphi: \Phi^{+}\right) \delta\left(\Phi^{+}, \varphi, h\right) .
$$

Thus by Lemma 7.8, for any choice $\Psi^{+}$of positive roots for $\Psi$,

$$
\sum_{\varphi \in \mathcal{T}\left(\Phi, \psi_{t}\right)} \epsilon\left(\varphi: \Phi^{+}\right) \delta\left(\Phi^{+}, \varphi, h\right)=\sum_{\psi_{I} \in \mathcal{T}\left(\Phi_{I}\right)} \epsilon\left(\psi_{I} \cup \psi_{t}: \Psi^{+}\right) \delta\left(\Psi^{+}, \psi_{I} \cup \psi_{t}, h\right) .
$$

By Lemma 7.9, we can choose $\Psi^{+}(h)$ so that $\Psi^{+}(h) \cap \Phi_{t}=\Phi_{R}^{+}(h)$ and for all $\psi_{I} \in \mathcal{T}\left(\Phi_{I}\right)$,

$$
\delta\left(\Psi^{+}(h), \psi_{I} \cup \psi_{t}, h\right)=(-1)^{r\left(\Phi_{I}\right)} .
$$

Write $\Phi_{I}^{+}(h)=\Phi_{I} \cap \Psi^{+}(h)$. Using this choice of $\Psi^{+}(h)$,

$$
\begin{gathered}
\sum_{\varphi \in \mathcal{T}\left(\Phi, \psi_{t}\right)} \epsilon\left(\varphi: \Phi^{+}\right) \delta\left(\Phi^{+}, \varphi, h\right)=(-1)^{r\left(\Phi_{I}\right)} \sum_{\psi_{I} \in \mathcal{T}\left(\Phi_{I}\right)} \epsilon\left(\psi_{I} \cup \psi_{t}: \Psi^{+}(h)\right) \\
=(-1)^{r\left(\Phi_{I}\right)} \epsilon\left(\psi_{t}: \Phi_{R}^{+}(h)\right) \sum_{\psi_{I} \in \mathcal{T}\left(\Phi_{I}\right)} \epsilon\left(\psi_{I}: \Phi_{I}^{+}(h)\right)=\epsilon\left(\psi_{t}: \Phi_{R}^{+}(h)\right)
\end{gathered}
$$

by Theorem 5.5 .

\section{REFERENCES}

[1] Harish-Chandra, Discrete series for semisimple Lie groups I, Acta Math., 113 (1965), 241318. MR 36:2744

[2] Harish-Chandra, Harmonic analysis on real reductive groups I, J. Funct. Anal., 19 (1975), 104-204. MR 53:3201

[3] R. Herb, Fourier inversion and the Plancherel theorem, (Proc. Marseille Conf. 1980), Lecture Notes in Math. Vol. 880, Springer-Verlag, Berlin and New York, 1981, 197-210. MR 83f:22013

[4] R. Herb, The Plancherel theorem for semisimple Lie groups without compact Cartan subgroups, (Proc. Marseille Conf. 1982), Lecture Notes in Math. Vol. 1020, Springer-Verlag, Berlin and New York, 1983, 73-79. MR 85a:22001 
[5] R. Herb, Discrete series characters and two-structures, Trans. AMS, 350 (1998), 3341-3369. MR 98k:22058

[6] R. Herb, Stable discrete series characters as lifts from complex two-structure groups, Pacific J. Math. 196 (2000), 187-212. CMP 2001:04

[7] R. Herb, Two-structures and discrete series character formulas, Proceedings of Symposia in Pure Mathematics, Vol. 68, A.M.S. Providence RI, 2000, 285-319. CMP 2000:16

[8] R. Herb and J.A. Wolf, The Plancherel theorem for general semisimple groups, Compositio Math., 57 (1986), 271-355. MR 87h:22020

[9] A.W. Knapp, Representation Theory of Semisimple Groups, An Overview Based on Examples, Princeton U. Press, Princeton, N.J., 1986. MR 87j:22022

[10] D. Shelstad, Orbital integrals and a family of groups attached to a real reductive group, Ann. Sci. Ecole Norm. Sup. 12 (1979), 1-31. MR 81k:22014

[11] D. Shelstad, Embeddings of L-groups, Canad. J. Math. 33 (1981), 513-558. MR 83e:22022

[12] D. Shelstad, L-indistinguishability for real groups, Math. Ann. 259(1982), 385-430. MR 84c:22017

[13] J.A. Wolf, Unitary representations on partially holomorphic cohomology spaces, Memoirs AMS, 138 (1974). MR 56:3194

Department of Mathematics, University of Maryland, College Park, Maryland 20742

E-mail address: rah@math.umd.edu 